\title{
Aeroelasticity of Composite Plate Wings using HSDT and Higher- Order FEM
}

Justin A. Haught

West Virginia University, jnhaught@mix.wvu.edu

Follow this and additional works at: https://researchrepository.wvu.edu/etd

Part of the Aerodynamics and Fluid Mechanics Commons, and the Structures and Materials

\section{Commons}

\section{Recommended Citation}

Haught, Justin A., "Aeroelasticity of Composite Plate Wings using HSDT and Higher-Order FEM" (2020). Graduate Theses, Dissertations, and Problem Reports. 7547.

https://researchrepository.wvu.edu/etd/7547

This Thesis is protected by copyright and/or related rights. It has been brought to you by the The Research Repository @ WVU with permission from the rights-holder(s). You are free to use this Thesis in any way that is permitted by the copyright and related rights legislation that applies to your use. For other uses you must obtain permission from the rights-holder(s) directly, unless additional rights are indicated by a Creative Commons license in the record and/ or on the work itself. This Thesis has been accepted for inclusion in WVU Graduate Theses, Dissertations, and Problem Reports collection by an authorized administrator of The Research Repository @ WVU. For more information, please contact researchrepository@mail.wvu.edu. 


\title{
Aeroelasticity of Composite Plate Wings using HSDT and Higher-Order FEM
}

\author{
Justin A. Haught
}

Thesis submitted to the

Benjamin M. Statler College of Engineering and Mineral Resources

at West Virginia University

in partial fulfillment of the requirements for the degree of

\author{
Master of Science \\ in \\ Aerospace Engineering \\ Nithi Sivaneri, Ph.D., Chair \\ Ever Barbero, Ph.D. \\ Christopher Griffin, Ph.D.
}

Department of Mechanical and Aerospace Engineering

Morgantown, West Virginia

May 2020

Keywords: Aeroelasticity, Composite plate, Flutter, Divergence, Shear deformation theory, Finite element modeling

Copyright (C) 2020 Justin Haught 


\title{
Abstract \\ Aeroelasticity of Composite Plate Wings using HSDT and Higher-Order FEM
}

\author{
Justin A. Haught
}

The aeroelasticity of composite wings is becoming an increasingly researched topic in aircraft design, as designers continue to replace aluminum alloy components with those made of composite materials because of their favorable strength-to-weight ratio, fatigue characteristics, and corrosion resistance. Additionally, the bending-torsion coupling exhibited by composite laminates readily allow for the aeroelastic optimization of an aerodynamic structure through the process of aeroelastic tailoring. Wings made of composites materials, however, are more vulnerable to shear deformation.

The objective of the present research is to study the divergence and flutter characteristics of composite plate wings using a higher-order shear deformation theory (HSDT) with higher-order finite element modeling (FEM). This is expected to clarify whether HSDT offers any meaningful advantage over the more widely used first order shear deformation theory (FSDT) and to determine how the application of an $h-p$ version conforming plate element improves computational aeroelastic modeling.

A new higher-order composite plate element, called MONNA, is introduced and coupled with the vortex lattice, doublet lattice, and doublet point aerodynamic panel method models. The novel element is conforming, but it does not directly have the capacity for quadrilateral transformation. The higher-order plate element demonstrates better aeroelastic convergence and accuracy relative to traditional elements. Negligible difference is shown between FSDT and HSDT for a variety of plate materials and thicknesses. A parametric study is performed, revealing the divergence-flutter tradeoff associated with change in ply orientation along with other insights into aeroelastic tailoring. Overall, the HSDT is concluded to be superfluous in aeroelastic plate analysis. The practicality of the new higher-order element is found to be limited in the field of aeroelasticity, but it may be more practical in other engineering applications. 


\section{Acknowledgements}

There are a number a individuals I would like to thank for their support during the completion of this thesis. First and foremost, I express my sincere gratitude to my research advisor and committee chair, Dr. Nithi Sivaneri, for his continual and invaluable guidance during my time as a graduate student. Dr. Sivaneri has demonstrated a selfless devotion to my success and a high level of patience with my stubborn personality. He is one of the greatest teachers I have ever had and has guided me to be a better engineer, researcher, and overall professional.

Dr. Sivaneri invited me to join him as a graduate assistant in Dayton, $\mathrm{OH}$ for the Air Force Summer Faculty Fellowship Program (SFFP) at Wright-Patterson Air Force Base, where we were advised by Dr. Caleb Barnes. I sincerely thank Dr. Barnes for working closely with us over the summer and always being willing to support us with any problems we encountered. I also thank the Air Force SFFP for giving us the opportunity to conduct research at such a wonderful research facility and for funding a portion of this research.

I also express my gratitude to Dr. Ever Barbero and Dr. Christopher Griffin for their support and valuable suggestions as committee members. The WVU AIAA outreach events to local elementary schools, of which Dr. Griffin has led, are some of the highlights of my university experience.

My acknowledgments are not complete without considering my family and friends. My parents, Brian and Robin Haught, have supported me unconditionally in everything I have done and have provided me with a firm foundation on which to grow and develop as a person. This thesis is dedicated to them. Additionally, I would like to recognize my best friend since pre-school, Nathan Rhine, who has always been there for me as a genuine friend and authentic person. Finally, I thank God, whose illogical insistence to love others more than ourselves continues to give me life. 


\section{Contents}

List of Tables $\quad$ vi

List of Figures vii

Abbreviations $\quad$ ix

List of Symbols $\quad \mathrm{x}$

1 Introduction $\quad 1$

1.1 Problem Statement . . . . . . . . . . . . . . . . . . . . . 1

1.2 Literature Review . . . . . . . . . . . . . . . . . . . . . 3

1.3 Need for Present Research . . . . . . . . . . . . . . . 5

1.4 Objectives . . . . . . . . . . . . . . . . 6

1.5 Thesis Outline . . . . . . . . . . . . . . . . . . 7

2 Structural Models $\quad 8$

2.1 Introduction . . . . . . . . . . . . . . . . . . 8

2.2 Theoretical Formulation . . . . . . . . . . . . . . . . . . 9

2.2.1 Composite Plate Theories . . . . . . . . . . . . . . . 9

2.2.2 Constitutive Equations for HSDT Composite Laminate . . . . . . 14

2.2.3 Hamilton's Principle . . . . . . . . . . . . . . . . . . 17

2.2.4 Virtual Strain Energy _. . . . . . . . . . . . . . . . 17

2.2.5 Virtual Kinetic Energy . . . . . . . . . . . . . . . . . . 18

2.3 Finite Element Formulation . . . . . . . . . . . . . . . . . . . . 21

2.3 .1 Introduction . . . . . . . . . . . . . . . . 21

2.3.2 MONNA Plate Element . . . . . . . . . . . . . . . . 22

2.3.3 Coordinate Transformation . . . . . . . . . . . . . . . 22

2.3.4 Shape Functions . . . . . . . . . . . . . . . . . . 26

2.3.5 MONNA Plate Transformation . . . . . . . . . . . . . . 30

2.3.6 Element Stiffness Matrix Formulation . . . . . . . . . . . . . . 33

2.3.7 Element Inertia Matrix Formulation . . . . . . . . . . . . . . . . . 35

2.3.8 Traditional Plate Elements . . . . . . . . . . . . . . . . 36

3 Aerodynamic Models $\quad 38$

3.1 Introduction . . . . . . . . . . . . . . . . . . . . . . . . . . . . . . . . . .

3.2 Steady Aerodynamics . . . . . . . . . . . . . . . . . . . . . . . . . . . . . . . . . 39

3.2 .1 Vortex Lattice Method . . . . . . . . . . . . . . . . . . . . 39

3.3 Unsteady Aerodynamics . . . . . . . . . . . . . . . . . . . 41

3.3.1 Doublet Lattice Method . . . . . . . . . . . . . . . . . . . 41 
3.3.2 Doublet Point Method . . . . . . . . . . . . . . . . . . 46

4 Aeroelaticity Models $\quad 49$

4.1 Introduction . . . . . . . . . . . . . . . . . . . . . . 49

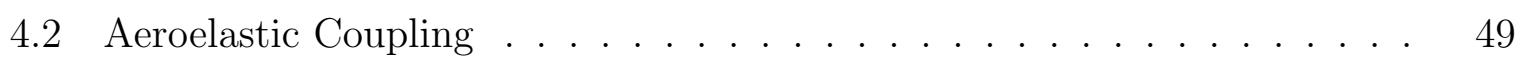

4.3 Divergence . . . . . . . . . . . . . . . . . . . 52

4.4 Flutter . . . . . . . . . . . . . . . . . . . 54

5 Numerical Verification $\quad 57$

5.1 Structural Model Verification _. . . . . . . . . . . . . . . 57

5.1 .1 Isotropic Plates . . . . . . . . . . . . . . . . . . . 57

5.1 .2 Composite Plates . . . . . . . . . . . . . . . . . . . . . 59

5.2 Aerodynamic Model Verification . . . . . . . . . . . . . . . . 61

5.2 .1 Vortex Lattice Method . . . . . . . . . . . . . . . . . . . . . 61

5.2 .2 Doublet Lattice Method . . . . . . . . . . . . . . . . . . 62

5.2 .3 Doublet Point Method . . . . . . . . . . . . . . . . 63

5.3 Aeroelasticity Verification . . . . . . . . . . . . . . . . . 65

$5.3 .1 \quad$ Isotropic Plate . . . . . . . . . . . . . . . . 65

5.3 .2 Composite Plates . . . . . . . . . . . . . . . 66

6 Results and Discussion $\quad 70$

6.1 Aeroelastic Convergence Study . . . . . . . . . . . . . . . . . 70

6.2 Effect of HSDT and Laminate Thickness . . . . . . . . . . . . . . . 75

6.3 Effect of Ply Orientation . . . . . . . . . . . . . . . . . 81

6.4 Effect of Aspect Radio . . . . . . . . . . . . . . . . . . . . . . . . . . 84

6.5 Effect of Composite Moduli Ratios . . . . . . . . . . . . . . 86

7 Conclusions and Recommendations $\quad 89$

$\begin{array}{ll}\text { References } & 91\end{array}$

$\begin{array}{ll}\text { A Gauss Quadrature Integration } & 94\end{array}$ 


\section{List of Tables}

3.1 Laschka coefficients for approximation of $I_{0} \ldots \ldots \ldots 44$

5.1 Maximum deflection and slope of square isotropic plate . . . . . . . 58

5.2 Natural frequencies of square isotropic plate $(\mathrm{rad} / \mathrm{s}) \ldots \ldots . . . .58$

5.3 Nondimensional deflection of square $[0 / 90]_{5}$ composite plate . . . . . . 59

5.4 Nondimensional fundamental natural frequency of square $[0 / 90]_{5}$ composite plate . . . . . . . . . . . . . . . 60

5.5 Nondimensional vortex strengths and lift coefficient using VLM . . . . 61

5.6 Complex pressure coefficients verification using DLM . . . . . . . . . 62

5.7 Divergence verification of isotropic plate wings . . . . . . . . . . 65

5.8 Flutter verification of isotropic plate wings . . . . . . . . . . . 66

5.9 Divergence verification of composite plate wings . . . . . . . . . . 67

5.10 Flutter verification of composite plate wings . . . . . . . . . 67

6.1 Convergence study for $\left[0_{2} / 90\right]_{s}$ laminate $(8 \times 12$ DPM elements $) \ldots . . .70$

6.2 Convergence study for $[0 / 90]_{3}$ laminate $(8 \times 12$ DPM elements $) \ldots . . .71$

6.3 Convergence study for $\left[45_{2} / 0\right]_{s}$ laminate $(8 \times 12$ DPM elements $) \ldots . . .72$

6.4 Convergence study for $[0 / 45]_{3}$ laminate $(8 \times 12$ DPM elements $) \quad \ldots . . .72$

6.5 Convergence study for $[45 /-45 / 0]_{\mathrm{s}}$ laminate $(8 \times 12$ DPM elements $) \ldots . .73$

6.6 Composite material properties for laminate thickness and HSDT analysis 75

6.7 Divergence speed analysis with $\left[0_{2 n} / 90_{n}\right]_{s}$ laminate $\ldots \ldots \ldots$. . . . 76

6.8 Divergence speed analysis with $\left[-45_{2 n} / 0_{n}\right]_{s}$ laminate $\ldots \ldots \ldots$. . . . 77

6.9 Flutter speed analysis with $\left[0_{2 n} / 90_{n}\right]_{s}$ laminate . . . . . . . . . 78

6.10 Flutter speed analysis with $\left[45_{2 n} / 0_{n}\right]_{s}$ laminate . . . . . . . . . 79

6.11 Composite plate properties for ply orientation analysis . . . . . . . 81

A.1 Gauss quadrature points and weights . . . . . . . . . . . . . . . 94 


\section{List of Figures}

1.1 Traditional wing bending-torsional coupling as per Ref.[3] . . . . . . . . . 2

1.2 Grumman X-29 experimental aircraft . . . . . . . . . . . . . . . 3

2.1 CLPT deformed geometry as per Ref. [18] . . . . . . . . . . . . . 10

2.2 FSDT deformed geometry as per Ref. [18] . . . . . . . . . . . . 11

2.3 Transverse normal deformations for CLPT, FSDT, and HSDT as per Ref.

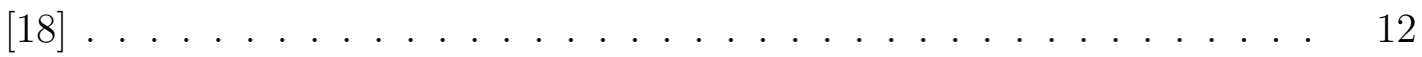

2.4 MONNA plate element with physical DOF distribution . . . . . . . . 22

2.5 Physical quadrilateral . . . . . . . . . . . . . . . . . . . 22

2.6 A Melosh HSDT plate element with DOF distribution . . . . . . . . . 36

2.7 A BFS HSDT plate element with DOF distribution . . . . . . . . . . 37

3.1 Vortex lattice method receiving and sending panels . . . . . . . . . . . 39

3.2 Doublet lattice method receiving and sending panels . . . . . . . . . . . . 41

3.3 Doublet point method receiving and sending panels . . . . . . . . . 46

4.1 Aeroelasticity coupling illustration . . . . . . . . . . . . . . . 50

5.1 FEM boundary conditions for plate simply supported on all edges . . . 58

5.2 Vortex lattice method verification configuration [23] . . . . . . . . . 61

5.3 Doublet lattice method verification configuration . . . . . . . . . . . . 62

5.4 Spanwise pressure coefficient comparison using the DPM . . . . . . . 63

5.5 Chordwise real pressure coefficient comparison using the DPM . . . . . . 64

5.6 Chordwise imaginary pressure coefficient comparison using the DPM . . 64

5.7 Isotropic plate geometry . . . . . . . . . . . . . . . 65

5.8 Composite verification plate geometry . . . . . . . . . . 66

$5.9 V-g$ plot for $\left[0_{2} / 90\right]$ laminate using the DPM . . . . . . . . . 68

$5.10 V-\omega$ plot for $\left[0_{2} / 90\right]$ laminate using the DPM $\ldots \ldots \ldots \ldots$

6.1 Convergence study of $\left[0_{2} / 90\right]_{s}$ laminate . . . . . . . . . . . . . 71

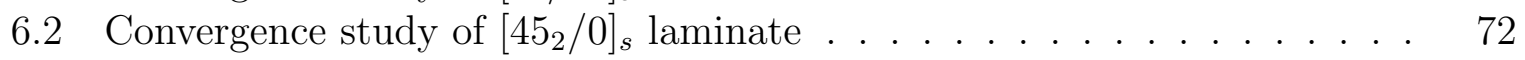

6.3 Convergence study of $[0 / 45]_{3}$ laminate $\ldots \ldots \ldots \ldots$

6.4 Convergence study of $[45 /-45 / 0]_{\mathrm{s}}$ laminate $\ldots \ldots \ldots \ldots$. . . . . . 74

6.5 Composite plate geometry for laminate thickness and HSDT analysis . . 75

6.6 HSDT divergence speed analysis with $\left[0_{2 n} / 90_{n}\right]_{s}$ laminate . . . . . . . 76

6.7 HSDT divergence speed analysis with $\left[-45_{2 n} / 0_{n}\right]_{s}$ laminate $\ldots \ldots . . . .67$

6.8 HSDT flutter speed analysis with $\left[0_{2 n} / 90_{n}\right]_{s}$ laminate . . . . . . . . 78

6.9 HSDT flutter speed analysis with $\left[45_{2 n} / 0_{n}\right]_{s}$ laminate $\ldots \ldots . . . . . \quad 79$

6.10 Nondimensional flutter and divergence speeds vs. ply orientation for $\left[\theta_{2} / 0\right]_{s}$ laminate . . . . . . . . . . . . . . . . . . . 82 
6.11 Nondimensional flutter and natural frequencies vs. ply orientation for $\left[\theta_{2} / 0\right]_{s}$ laminate . . . . . . . . . . . . . . . . 83

6.12 Divergence speeds vs. aspect ratio for $\left[-45_{2} / 0\right]_{s}$ and $[0 /-45]_{3}$ laminates . . 84

6.13 Flutter speeds vs. aspect ratio for $\left[45_{2} / 0\right]_{s}$ and $[0 / 45]_{3}$ laminates . . . . . 85

6.14 Divergence speeds vs. $E_{1} / E_{2}$ for various laminates . . . . . . . . 86

6.15 Divergence speeds vs. $G_{12} / E_{2}$ for various laminates . . . . . . . . . 87

6.16 Flutter speeds vs. $E_{1} / E_{2}$ for various laminates . . . . . . . . . . . . 88

6.17 Flutter speeds vs. $G_{12} / E_{2}$ for various laminates . . . . . . . . . . 88 


\section{Abbreviations}

$\begin{array}{ll}\text { AIC } & \text { Aerodynamic Influence Coefficients } \\ \text { BFS } & \text { Bogner, Fox, and Schmidt } \\ \text { CFD } & \text { Computational Fluid Dynamics } \\ \text { CLPT } & \text { Classical Laminate Plate Theory } \\ \text { DLM } & \text { Doublet Lattice Method } \\ \text { DOF } & \text { Degree of freedom } \\ \text { DPM } & \text { Doublet Point Method } \\ \text { FEM } & \text { Finite Element Modeling } \\ \text { FSDT } & \text { First-Order Shear Deformation Theory } \\ \text { HSDT } & \text { Higher-Order Shear Deformation Theory } \\ \text { IC } & \text { Influence Coefficients } \\ \text { MONNA } & \text { Multi-Order Nine-Node Advanced } \\ \text { SFFP } & \text { Summer Faculty Fellowship Program } \\ \text { VLM } & \text { Vortex Lattice Method }\end{array}$




\section{List of Symbols}

\begin{tabular}{|c|c|c|}
\hline Symbol & Units & Description \\
\hline$A$ & $\mathrm{~m}^{2}$ & plate surface area \\
\hline$[A]$ & $\mathrm{N} / \mathrm{m}$ & extension stiffness matrix \\
\hline$\left[A_{d}\right]$ & - & divergence aerodynamic influence coefficient matrix \\
\hline$\left[A_{f}\right]$ & - & flutter aerodynamic influence coefficient matrix \\
\hline$A_{m n}$ & $1 / \mathrm{m}$ & vortex lattice influence coefficients \\
\hline$A R$ & - & aspect ratio \\
\hline$b$ & $\mathrm{~m}$ & wing half-chord \\
\hline$B$ & $\mathrm{~m}$ & doublet point kernel function subcomponent \\
\hline$[B]$ & $\mathrm{N}$ & bending-extension coupling stiffness matrix \\
\hline$c$ & $\mathrm{~m}$ & wing chord \\
\hline$c_{1}$ & $1 / \mathrm{m}^{2}$ & tracer \\
\hline$C_{L}$ & - & lift coefficient \\
\hline$C_{p}$ & - & pressure coefficient \\
\hline$[C]$ & $\mathrm{Ns} / \mathrm{m}$ & artificial structural damping matrix \\
\hline$[D]$ & $\mathrm{Nm}$ & bending stiffness matrix \\
\hline$D_{i j}$ & $\mathrm{~m} / \mathrm{s}$ & unsteady influence coefficients \\
\hline$e$ & $\mathrm{~m}$ & doublet lattice panel half-span \\
\hline$E$ & $\mathrm{GPa}$ & isotropic elastic modulus \\
\hline$E_{1}$ & GPa & longitudinal elastic modulus \\
\hline$E_{2}$ & $\mathrm{GPa}$ & transverse elastic modulus \\
\hline$[E],[F],[H]$ & $\mathrm{Nm}^{2}, \mathrm{Nm}^{3}, \mathrm{Nm}^{5}$ & higher-order stiffness matrices \\
\hline$g$ & - & structural damping coefficient \\
\hline$G_{12}, G_{13}$ & GPa & in-plane shear moduli \\
\hline$G_{23}$ & $\mathrm{GPa}$ & transverse shear modulus \\
\hline$h$ & $\mathrm{~m}$ & laminate height \\
\hline$\left[H_{L}\right]$ & - & Lagrange shape function \\
\hline$[H]$ & - & Hermite shape function in physical domain \\
\hline$[\bar{H}]$ & - & Hermite shape function in standard domain \\
\hline$I_{0}, I_{1}, I_{2}$ & - & inertia terms \\
\hline$I_{3}, I_{4}, I_{6}$ & - & higher-order inertia terms \\
\hline$J$ & $\mathrm{~m}^{2}$ & Jacobian \\
\hline
\end{tabular}




\begin{tabular}{|c|c|c|}
\hline$[J]$ & $\mathrm{m}$ & Jacobian matrix \\
\hline$k$ & - & reduced frequency \\
\hline$K$ & - & kernel function \\
\hline$K_{s}$ & - & shear correction factor \\
\hline$K_{1}, K_{2}$ & - & kernel function subcomponents \\
\hline$\left[k_{e}\right]$ & $\mathrm{N} / \mathrm{m}$ & finite element stiffness matrix \\
\hline$l$ & $\mathrm{~m}$ & doublet line \\
\hline$L$ & $\mathrm{~N}$ & aerodynamic load \\
\hline$M$ & - & Mach number \\
\hline$[M]$ & $\mathrm{kg}$ & finite element inertia matrix \\
\hline$M_{x}, M_{y}, M_{x y}$ & $\mathrm{~N}$ & in-plane moment resultants \\
\hline$N$ & - & bilinear shape function \\
\hline$N_{a}$ & - & aerodynamic panels per structural element \\
\hline$N_{x}, N_{y}, N_{x y}$ & $\mathrm{~N} / \mathrm{m}$ & in-plane force resultants \\
\hline$P$ & $\mathrm{~N} / \mathrm{m}^{2}$ & pressure \\
\hline$P_{x}, P_{y}, P_{x y}$ & $\mathrm{Nm}^{2}$ & higher-order stress resultants \\
\hline$q_{\infty}$ & $\mathrm{m} / \mathrm{s}$ & freestream dynamic pressure \\
\hline$\{q\}$ & $\mathrm{m}$ & finite element displacement vector \\
\hline$\{Q\}$ & - & finite element load vector \\
\hline$Q_{x}, Q_{y}$ & $\mathrm{~N} / \mathrm{m}$ & transverse force resultants \\
\hline $\bar{Q}_{i j}^{(k)}$ & $\mathrm{N} / \mathrm{m}^{2}$ & transformed reduced stiffness matrix of $k^{t h}$ layer \\
\hline$R_{x}, R_{y}$ & $\mathrm{Nm}$ & higher-order stress resultants \\
\hline$s$ & $\mathrm{~m}$ & wing half-span \\
\hline$S_{w}$ & $\mathrm{~m}^{2}$ & total wing area \\
\hline$t$ & $\mathrm{~m}$ & laminate thickness \\
\hline$t_{p}$ & $\mathrm{~m}$ & ply thickness \\
\hline$T$ & $\mathrm{Nm}$ & kinetic energy \\
\hline$\left[T_{A}\right]$ & - & inverse second derivative transformation \\
\hline$\left[T_{1}\right]$ & - & aeroelastic deformation transformation \\
\hline$\left[T_{2}\right]$ & - & aeroelastic load transformation \\
\hline$u, v$ & $\mathrm{~m}$ & in-plane displacements in $\mathrm{x}$ and $\mathrm{y}$ \\
\hline$U$ & $\mathrm{Nm}$ & strain energy \\
\hline$U_{D}$ & $\mathrm{~m} / \mathrm{s}$ & divergence speed \\
\hline$U_{F}$ & $\mathrm{~m} / \mathrm{s}$ & flutter speed \\
\hline$U_{R}$ & $\mathrm{~m} / \mathrm{s}$ & reference aeroelastic speed \\
\hline$U_{\infty}$ & $\mathrm{m} / \mathrm{s}$ & freestream velocity \\
\hline$v$ & $\mathrm{~m}$ & $\mathrm{y}$-direction displacement \\
\hline$V$ & $\mathrm{~m}^{3}$ & plate volume \\
\hline$w_{b}, w_{s}$ & $\mathrm{~m}$ & bending and shear lateral displacement \\
\hline$w_{m}$ & $\mathrm{~m} / \mathrm{s}$ & downwash \\
\hline
\end{tabular}




\begin{tabular}{|c|c|c|}
\hline $\bar{w}_{i}$ & - & nondimensional normalwash \\
\hline$w$ & $\mathrm{~m}$ & general lateral displacement \\
\hline$W$ & $\mathrm{Nm}$ & work done \\
\hline$x_{0}, y_{0}$ & $\mathrm{~m}$ & doublet lattice delta coordinates \\
\hline$\alpha$ & - & angle of attack \\
\hline$\gamma$ & - & Euler's constant \\
\hline$\gamma_{x y}, \gamma_{y z}, \gamma_{x z}$ & - & transverse shear strains \\
\hline$\Gamma$ & $\mathrm{m}^{2} / \mathrm{s}$ & vortex strength \\
\hline$\delta()$ & - & variation of \\
\hline$\Delta_{j}$ & $\mathrm{~m}^{2}$ & nondimensional doublet point panel area \\
\hline$\epsilon_{x}, \epsilon_{y}, \epsilon_{z}$ & - & normal strains in $\mathrm{x}, \mathrm{y}$, and $\mathrm{z}$ \\
\hline$\eta$ & - & plate standard domain coordinate \\
\hline$\theta$ & - & composite ply orientation \\
\hline$\kappa$ & - & working form of kernel function \\
\hline$\lambda$ & - & eigenvalue \\
\hline$\lambda_{j}$ & deg & doublet line sweep angle \\
\hline$\Lambda_{L E}$ & $\operatorname{deg}$ & leading edge sweep angle \\
\hline$\nu$ & - & isotropic poisson ratio \\
\hline$\nu_{12}$ & - & in-plane poisson ratio \\
\hline$\xi$ & - & plate standard domain coordinate \\
\hline$\rho$ & $\mathrm{kg} / \mathrm{m}^{3}$ & plate density \\
\hline$\rho_{\infty}$ & $\mathrm{kg} / \mathrm{m}^{3}$ & freestream air density \\
\hline$\sigma_{x}, \sigma_{y}$ & $\mathrm{MPa}$ & normal stresses in $\mathrm{x}$ and $\mathrm{y}$ directions \\
\hline $\bar{\sigma}$ & - & nondimensional doublet point panel half-width \\
\hline$\tau_{x y}, \tau_{y z}, \tau_{x z}$ & $\mathrm{MPa}$ & transverse shear stresses \\
\hline$\phi_{x}, \phi_{x}$ & - & normal rotations about $\mathrm{x}$ and $\mathrm{y}$ \\
\hline$\omega$ & $\mathrm{rad} / \mathrm{s}$ & oscillating frequency \\
\hline$\omega_{R}$ & $\mathrm{rad} / \mathrm{s}$ & reference oscillating frequency \\
\hline$(\cdot)$ & - & partial derivative with respect to time \\
\hline$\left({ }^{*}\right)$ & - & second partial derivative with respect to time \\
\hline()$^{x}$ & - & partial derivative with respect to $\mathrm{x}$ \\
\hline()$^{x x}$ & - & second partial derivative with respect to $\mathrm{x}$ \\
\hline()$^{y}$ & - & partial derivative with respect to $\mathrm{y}$ \\
\hline()$^{y y}$ & - & second partial derivative with respect to y \\
\hline
\end{tabular}




\section{Chapter 1}

\section{Introduction}

\subsection{Problem Statement}

The aeroelasticity of composite wings is becoming an increasingly researched and important topic in aircraft design, as designers continue to replace aluminum alloy components with those made of composite materials because of their favorable strength-to-weight ratio, fatigue characteristics, and corrosion resistance. Although composites have been used in aircraft since the 1950's, the implementation of structural composites has been restricted over the past decades because of the costliness and complexity of composite material development and application. This has been especially the case in the realm of civil aircraft, where cost considerations are of higher priority than military aircraft. This trend is changing because of the increasing need for low-weight aircraft with the capacity for less fuel burn and emissions. This is most clearly demonstrated with the release of the Boeing 787 and Airbus 350 XWB, where about $50 \%$ of the aircrafts' structural weight, including the wings, comprises of advanced composites [1].

Among the various aeroelastic phenomena, divergence and flutter are arguably the most important. Divergence is the phenomenon where the moment due to aerodynamic forces overcomes the wing's structural restorative moment leading to structural failure. Flutter is the self-excited vibration of an elastic body where energy is effectively extracted from the air stream to produce sustained structural oscillation. Flutter occurs at a critical flow speed when aerodynamic forces associated with the motion of two or more vibrational modes couple together in an undesirable manner [2]. Although different combinations of vibrational modes may lead to flutter, the most critical mode combination in the context of aircraft wings is that between bending and torsion.

There are multiple sources of bending-torsion coupling that must be considered for composite wings. The traditional forms of coupling include inertial, aerodynamic, and elastic coupling. These types of coupling are present regardless whether the wing is made of metallic or composite structure. Inertial coupling, for example, is due to a 
non-coincident shear center and center of gravity. An example of traditional coupling is illustrated in Figure 1.1. When the wing undergoes upward bending motion, an inertial moment acts about the elastic axis, causing torsional rotation. The rotation induces increased lift, which then produces additional bending translation through aerodynamic coupling. The same phenomenon can occur for downward bending motion.

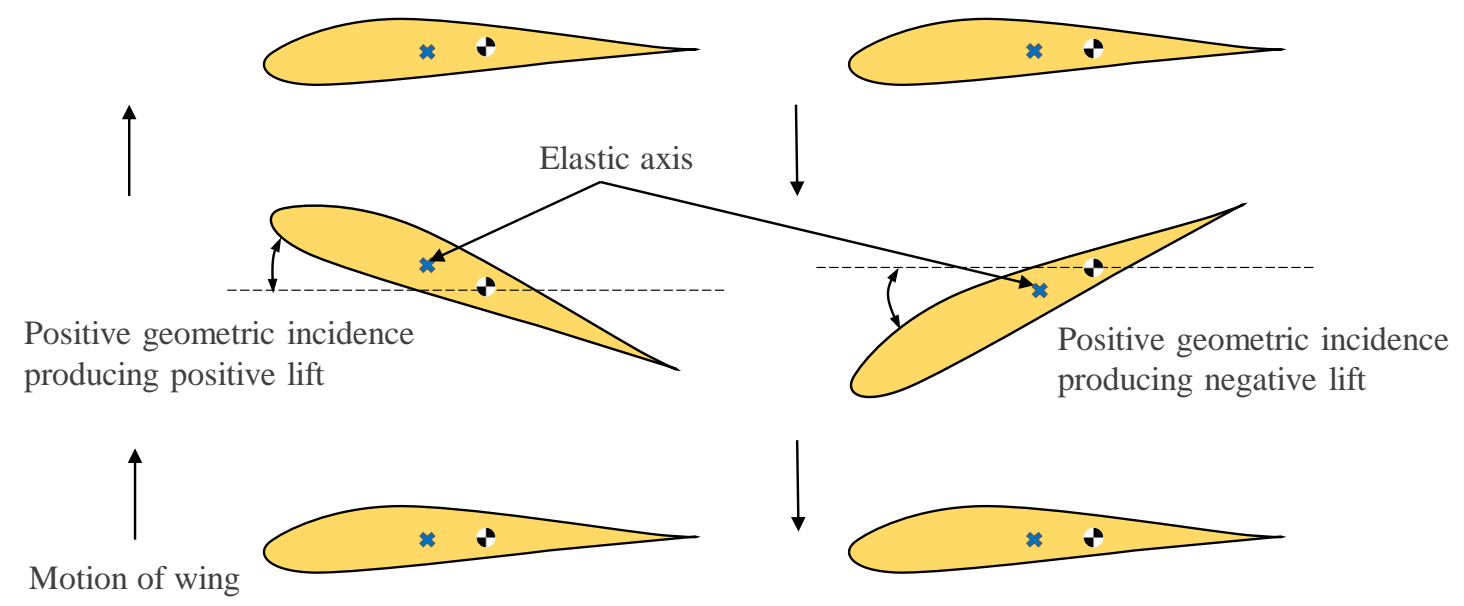

Figure 1.1: Traditional wing bending-torsional coupling as per Ref.[3]

The bending-torsion coupling that is unique to composite laminates is derived from their anisotropic properties. This coupling is referred to as material coupling and is present in laminates with non-zero $D_{16}$ and $D_{26}$ coefficients in the bending stiffness matrix. Consequentially, the total bending-torsional coupling in a composite wing is from inertial, aerodynamic, elastic, and material sources [4]. The presence of material coupling is beneficial, as it provides the designer with the freedom to manipulate the laminate to achieve an optimized structure. This is referred to as aeroelastic tailoring, which, for example, was utilized to avoid divergence in the Grumman X-29 shown in Figure 1.2.

Another complication associated with composite materials is their low transverse shear modulus relative to isotropic materials. While the shear modulus of metallic materials is $G_{m} \approx E_{m} / 2.5$, the in-plane shear modulus of composites is $G_{12} \approx$ $E_{1} / 25$, which implies that composites are more vulnerable to shear deformation.

The modeling of aeroelastic structure in research and the aeronautical industry is most commonly done with the finite element method, as this versatile approach can account for complicated geometries. Generally, the finite element solution approaches the exact solution by means of one of two methods. The $h$-version method increases solution accuracy by increasing the number of elements, while the $p$-version method increases solution accuracy by increasing the number of internal nodes. Another version combining the two methods, the $h-p$ version, uses both techniques. It is well established 
that the $h-p$ version offers the ability to achieve an exponential rate of convergence, as opposed to the algebraic rate of convergence found in the other versions [5]. Thus, there is potential to increase the rate of structural convergence in the context of aeroelastic analysis if $h-p$ elements are utilized.

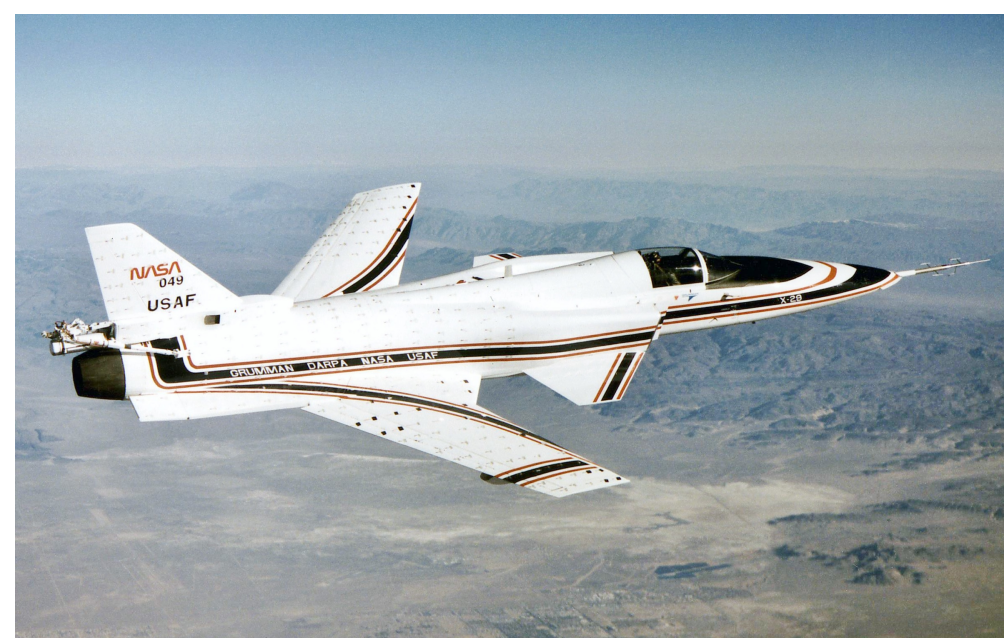

Figure 1.2: Grumman X-29 experimental aircraft

The flutter characteristics of isotropic wings have been established and are wellknown. Contrarily, the flutter behavior of composite wings remains a developing area of research because of their complexity and limited application. Thus, with the increasing demand to implement composites as aircraft structure, the opportunity to use composites for aeroelastic tailoring, and the relatively low shear moduli, there is a need to thoroughly investigate the aeroelasticity of composite wings while considering the effects of shear deformation and improvements to aeroelastic structural modeling.

\subsection{Literature Review}

A fair amount of literature has been published on the general aeroelasticity characteristics of composite wings and the benefits of aeroelastic tailoring. The present thesis is concerned specifically with the finite element modeling of aeroelastic composite wings and the effects of shear deformation, thus, this literature review primarily focuses on the existing aeroelastic studies that have implemented finite element models.

Hollowell and Dugundji [6] have performed analytical and experimental investigations into the aeroelastic characteristics of unswept, rectangular, cantilever composite plates made from graphite-epoxy for a variety of laminates. The analytical solution comprised of a Rayleigh-Ritz structural model and two-dimensional unsteady strip theory aerodynamics. Good agreement was demonstrated between the analytical and experimental results. These experimental results have become the baseline 
reference with which researchers have regularly validated computational composite aeroelasticity models over the last few decades.

Lin, Lu, and Tarn [7] have studied the flutter of cantilever composite plates with an 18 degree-of-freedom triangular finite element based on classical laminate plate theory (CLPT). The doublet lattice method (DLM) was chosen for the aerodynamic model and the surface spline method for aeroelastic coupling. Their analysis included how changing composite ply orientation, sweep angle, and aspect ratio influenced vibration and flutter speed. Overall, they have demonstrated the divergence-flutter trade-off associated with aeroelastic tailoring and concluded that composite materials have the potential to substantially improve aeroelastic characteristics, albeit through rigorous parametric investigation.

Koo and Lee [8] have studied the effect of structural damping on the aeroelasticity of composite plate wings. A nine-node isoparametric rectangular finite element with first-order shear deformation theory (FSDT) modeled the plate structure, the doublet point method (DPM) modeled the unsteady aerodynamics, and the surface spline method was used for coupling. The effect of composite ply orientation on flutter speed was studied for rectangular, swept-forward, and swept-back wings. Structural damping of composite materials slightly increased flutter speeds and decreased flutter frequencies.

Chattopadhyay, Zhang, and Iha [9] have performed a structural and aeroelastic analysis of a rectangular composite wing box with moderately thick walls using a higher-order shear deformation theory (HSDT). Thin-wall assumptions, which often involve CLPT or FSDT, have been demonstrated to be invalid for applications with moderately thick walls. For this reason, the higher order shear theory was implemented to account for an accurate variation of shear stresses and strains through the wall thickness. The wing box structure was modeled using plate finite elements, the aerodynamics was modeled with the DLM, and the flutter speeds were found using the $k$-method. Wing box flutter speeds were calculated for different ply orientations.

Koo [10] has investigated the aeroelastic characteristics of doubly-swept isotropic and composite wings for enhanced stability. The plate wing was modeled using four-node rectangular finite elements with FSDT, the aerodynamic loads were evaluated using the DPM, and the flutter speeds were found using the $k$-method. The results indicated that aeroelastic stability could be significantly improved by adjusting the inboard wing sweep angle.

Kameyama and Fukunaga [11] have performed an aeroelastic composite plate wing design optimization using lamination parameters. Four-node CLPT rectangular plate finite elements were employed to model the structure, while the DPM was used for unsteady aerodynamic loads. The effect of lamination configuration on divergence and flutter characteristic were first studied followed by a minimum weight design using a 
genetic algorithm. The optimization design study demonstrated the effectiveness of aeroelastic tailoring.

Abbas, Negm, and Elshafei [12] have studied the flutter and divergence characteristics of composite plate wings using an analytical Rayleigh-Ritz model with a modified version of the HSDT. The aerodynamic loads were evaluated using the DPM and the flutter speeds were calculated with the $k$-method. The effect of ply orientation on natural frequencies, divergence speeds, and flutter speeds were explored. The analytical results were in general agreement with experimental, analytical, and numerical sources.

Mahran, Negm, and Elsabbagh [13] have written a booklet on an aeroelastic analysis of plate wings using the finite element method, which explored a detailed formulation of their aeroelasticity model and solution procedure. Their structural plate model was based on CLPT nonconforming triangular plate-shell elements, whereas their steady and unsteady aerodynamics were solved with the vortex lattice method (VLM) and DLM, respectively. Flutter and divergence speeds for both isotropic and composite wings were calculated and found to be in very good agreement with experimental and computational results. Aeroelasticity results were then analyzed by varying fiber orientation, sweep angle, and taper ratio.

Mahran, Elsabbagh, and Negm [14] have also published an aeroelastic analysis comparing the performance of five different finite elements. The plate-shell elements included: (1) CLPT nonconforming three-node triangular element, (2) CLPT nonconforming four-node quadrilateral element, (3) CLPT nonconforming four-node quadrilateral element based on deformation modes, (4) CLPT nonconforming 8-node quadrilateral element, and (5) CLPT nonconforming 9-node quadrilateral element. The elements were compared by means of static, dynamic, and aeroelastic tests. They concluded the three-node triangular element provided the most accurate results but required significantly more computational time relative to the nine-node quadrilateral element, which also achieved a high level of accuracy.

\subsection{Need for Present Research}

Composite wings are generally more vulnerable to the effects of shear deformation relative to traditional metallic wings. The classical laminate plate theory (CLPT) neglects shear deformation and has been demonstrated to be inadequate for the analysis of moderately thick laminates [15]. The most common way of accounting for shear deformation is by applying the first-order shear deformation theory. This was done in the context of composite aeroelasticity by Ref. [8] and [10]. The FSDT is simple to implement and performs well for both thin and moderately thick laminates, however, the theory requires the use of shear correction factors, and the accuracy of the solution 
can depend on these factors. The issues associated with the FSDT can be overcome by utilizing a higher-order shear deformation theory (HSDT), which has no need for shear correction factors and is highly accurate [16]. The problem with higher-order shear deformation theories is that they often require $C^{1}$ continuity of lateral displacements, which can be complicated to implement.

Although Ref. [9] studied the aeroelasticity of a composite wing box using an HSDT, their results were never compared with those produced by FSDT such that their decision to apply HSDT instead of the less complicated FSDT was never ultimately justified. Reference [12] studied the aeroelasticity of plate wings using a modified HSDT, but neglected to provide a reason for the application of modified HSDT in the context of aeroelasticity. Because previous investigations have failed to justify the use of higher-order theory, there is a need to compare the aeroelasticity of composite wings between HSDT and FSDT while varying laminate thicknesses to clarify whether an HSDT offers any meaningful advantage over the simpler FSDT.

Improvements to structural modeling in aeroelasticity are always in demand. It has been demonstrated that $h-p$ version finite elements have potential to achieve significantly faster rates of convergence relative to conventional $h$-version elements. Reference [14] compared the aeroelasticity performance of $h-p$ version elements with conventional elements, however, all of the elements used in the study were nonconforming, which may have difficulty with structural convergence [17]. Thus, there is a need to determine the potential improvement to aeroelastic convergence using conforming $h-p$ version finite elements.

\subsection{Objectives}

The objectives of the present thesis are as follows:

1. Formulate a computational composite plate aeroelasticity model using higher-order finite elements with the capacity for FSDT and HSDT

2. Compare composite plate divergence and flutter speeds between HSDT and FSDT with varying laminate thickness to clarify the benefits of applying a higher-order shear deformation theory

3. Analyze the aeroelastic convergence of a conforming $h-p$ version plate element and compare its performance with traditional $h$-version plate elements

4. Investigate the effect of laminate thickness, ply orientation, aspect ratio, and moduli ratios on divergence and flutter speeds 


\subsection{Thesis Outline}

The structure of the thesis is organized as follows:

- Chapter 2 presents the theoretical and finite element formulation of the composite plate structure. Composite plate theories are first explored, followed by a detailed theoretical formulation of the higher-order shear deformation theory applied in this analysis. The finite element formulation introduces a new higher-order plate element, called MONNA, and describes its development.

- Chapter 3 presents the panel method aerodynamic models employed in the analysis. These include the steady vortex lattice method for divergence and the unsteady doublet lattice and doublet point methods for flutter. The unsteady models are defined in detail to provide the reader with a single reference that gives an in-depth comparison between the two methods.

- Chapter 4 explores the aeroelastic coupling methodology between the structural and aerodynamic models. Although the surface spline method is regularly used in computational research and industry, here, the finite element shape function method is presented.

- Chapter 5 documents the verification and validation of each structural, aerodynamic, and aeroelastic model. Each model is written from scratch in MATLAB such that this procedure is necessary to justify their application.

- Chapter 6 includes the results and discussion of the accomplished research. This involves a convergence study, a comparison in aeroelastic performance between HSDT and FSDT, and a parametric study.

- Chapter 7 offers the conclusions of the present analysis and recommendations for future research. 


\section{Chapter 2}

\section{Structural Models}

\section{$2.1 \quad$ Introduction}

There are multiple approaches available to model a continuous aeroelastic structure. These include the following methods [2]:

1. Analytical method by exactly solving the governing partial differential equations

2. Approximate method by using assumed shapes (i.e. Rayleigh-Ritz Method)

3. Approximate method by discretizing the system (i.e. Finite Element Method)

The analytical method works for simple systems, but it is not useful when analyzing complicated structures such as aircraft wings. The assumed shapes approach is employed more often and is still being used in aeroelastic research, however, this approach still suffers when handling complex geometry. [2]. The finite element method has proven to be the most powerful and versatile method to represent aeroelastic structures. This is primarily because of its capacity to model complex semi-monocoque structures with high accuracy.

Composite laminates usually have large planar dimensions relative to their thickness such that they are often treated as plates in the finite element method. Additionally, plate elements are suitable for representing thin wing structure and effectively couple with panel method aerodynamics for an aeroelastic analysis. For these reasons, finite element composite plates are utilized as the structural model in the present study. The current chapter explores the existing composite plate theories followed by a detailed formulation of the higher-order order shear theory used in this thesis and its finite element implementation. 


\subsection{Theoretical Formulation}

\subsubsection{Composite Plate Theories}

Among the composite plate theories, the Classical Laminate Plate Theory (CLPT) and the First-Order Shear Deformation Theory (FSDT) are the most common. In the present section, these theories are briefly discussed before a detailed formulation of the HigherOrder Shear Deformation Theory (HSDT) used in the structural model.

\section{Classical Laminate Plate Theory}

The classical laminated plate theory is the simplest of the composite plate theories. Here, the Kirchhoff hypothesis holds, which can be described as (with $x-y$ in the plane of the plate and $z$ in the transverse direction)

1. Straight lines perpendicular to the midsurface (transverse normals) remain straight after deformation.

2. Transverse normals do not elongate $\left(\epsilon_{z}=0\right)$.

3. Transverse normals rotate such that they remain perpendicular to the midsurface after deformation $\left(\gamma_{x z}=0, \gamma_{y z}=0\right)$.

There are additional assumptions and restrictions made in the formulation of the theory. These include:

- Layers are perfectly bonded together.

- Layer material is linearly elastic and orthotropic.

- Layers have uniform thickness.

- Strains and displacements are small.

- The transverse shear stresses are zero at the top and bottom of the laminate.

The CLPT model is illustrated in Figure 2.1. The displacement field is expressed as

$$
\begin{aligned}
u(x, y, z, t) & =u_{0}(x, y, t)-z \frac{\partial w_{0}}{\partial x} \\
v(x, y, z, t) & =v_{0}(x, y, t)-z \frac{\partial w_{0}}{\partial y} \\
w(x, y, z, t) & =w_{0}(x, y, t)
\end{aligned}
$$


where $u_{0}$ and $v_{0}$ are the in-plane displacements and $w_{0}$ is the lateral displacement [18]. Note that the lateral displacement is only made up of the bending component such that CLPT does not account for shear deformation.

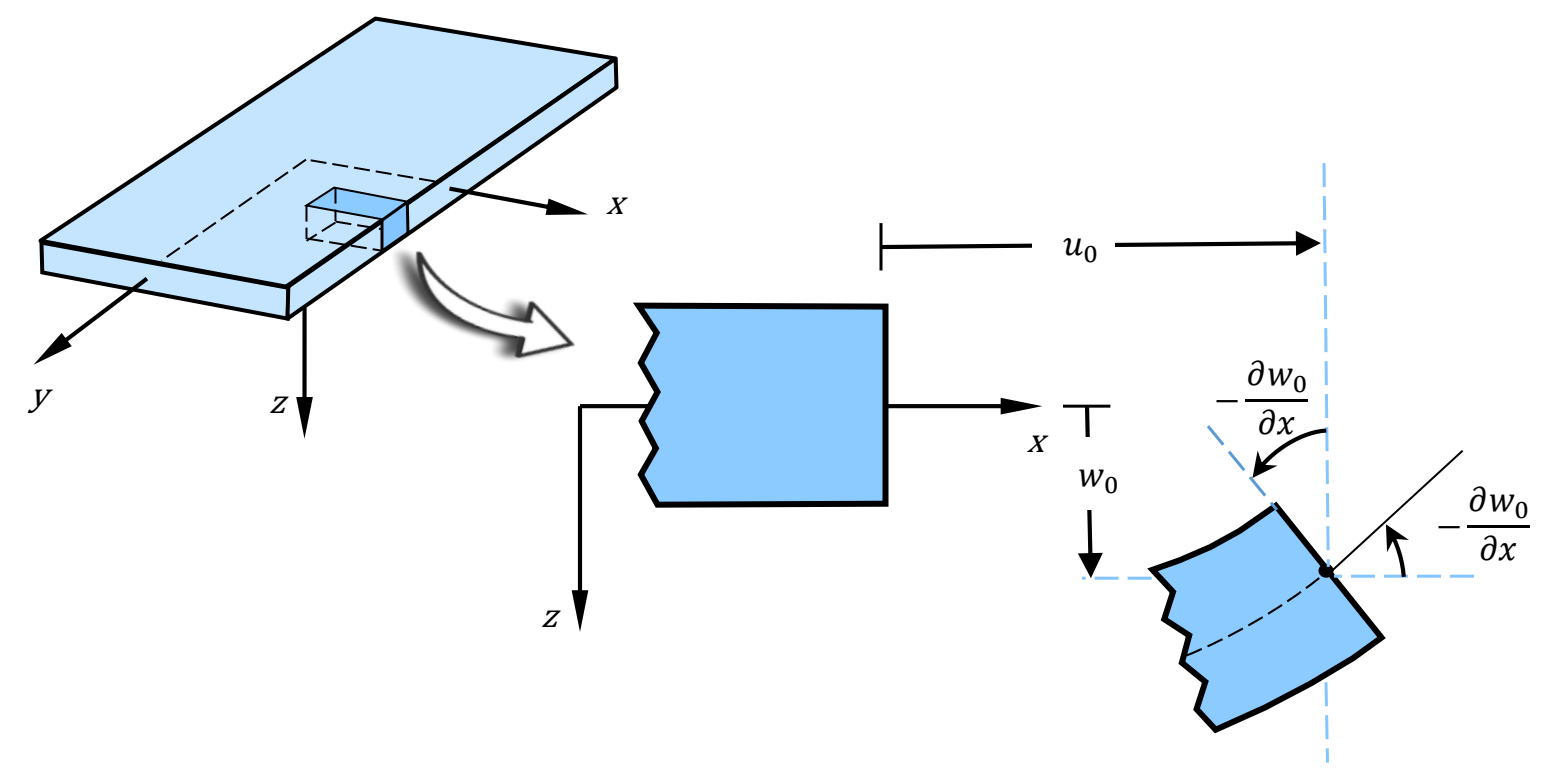

Figure 2.1: CLPT deformed geometry as per Ref. [18]

\section{First-Order Laminate Plate Theory}

In the first-order laminate plate theory, the of third component of the Kirchhoff hypothesis is not assumed. The transverse normals no longer remain perpendicular to the midsurface after deformation such that transverse shear strains are included in the theory. This is an important correction, as not including transverse shear strains can lead to quantifiable error in composite plates because of their low shear modulii. The plate geometry under the FSDT theory is illustrated in Figure 2.2. The displacement field for FSDT may represented as

$$
\begin{aligned}
u(x, y, z, t) & =u_{0}(x, y, t)+z \phi_{\mathrm{x}}(x, y, z) \\
v(x, y, z, t) & =v_{0}(x, y, t)+z \phi_{\mathrm{y}}(x, y, z) \\
w(x, y, z, t) & =w_{0}(x, y, t)
\end{aligned}
$$

where $\phi_{\mathrm{x}}$ and $\phi_{\mathrm{y}}$ are the transverse normal rotations with respect to the $y$ and $x$ axes, respectively. The lateral displacement is split into bending and shear components in the current formulation

$$
w(x, y, z, t)=w_{\mathrm{b}}(x, y, t)+w_{\mathrm{s}}(x, y, t)
$$




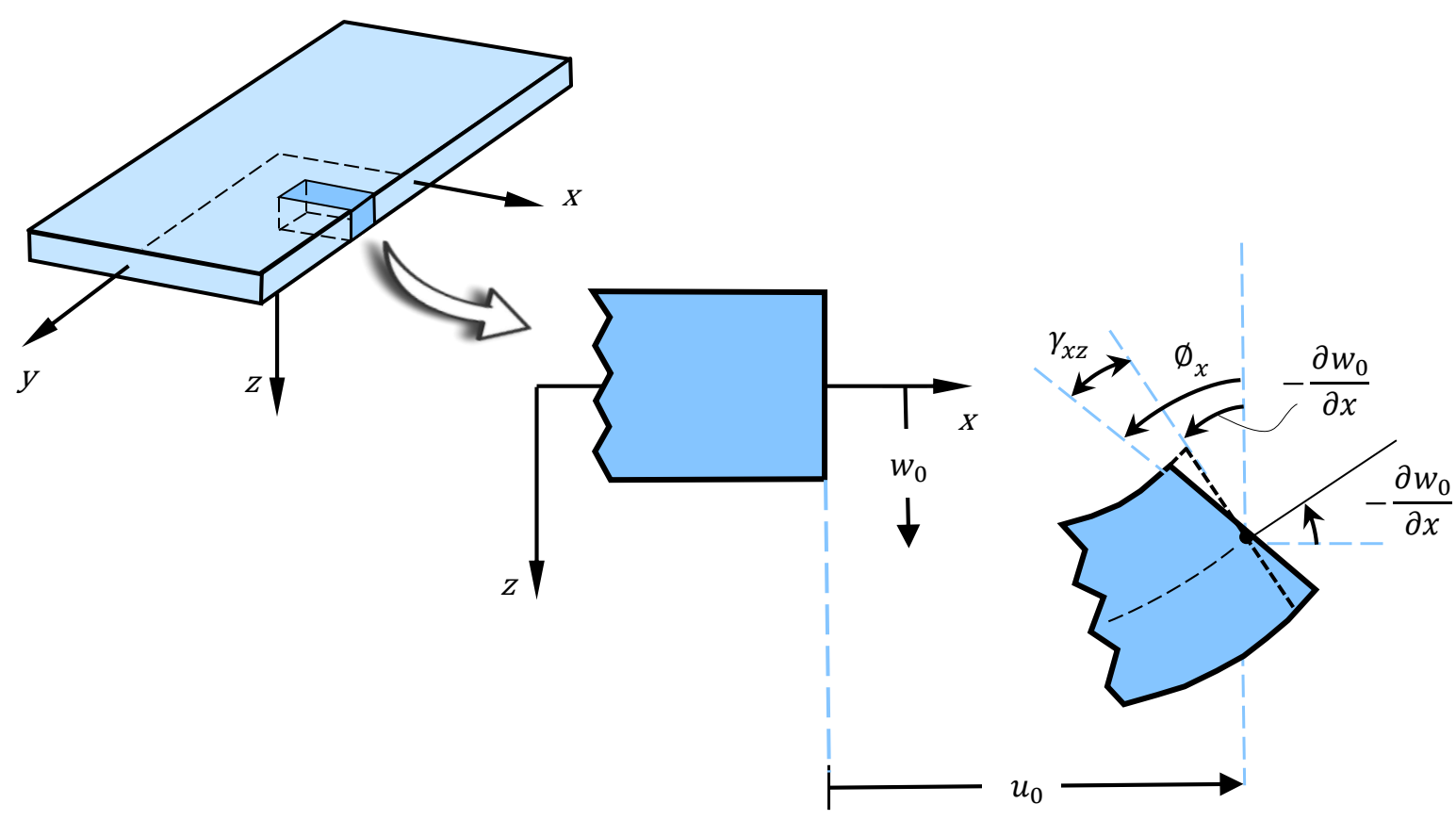

Figure 2.2: FSDT deformed geometry as per Ref. [18]

where $w_{\mathrm{b}}$ and $w_{\mathrm{s}}$ are the lateral displacements due to bending and shear, respectively. From Figure 2.3,

$$
\begin{aligned}
& \phi_{\mathrm{x}}=\gamma_{\mathrm{xz}}-\frac{\partial w_{0}}{\partial x} \\
& \phi_{\mathrm{y}}=\gamma_{\mathrm{yz}}-\frac{\partial w_{0}}{\partial y}
\end{aligned}
$$

where $\gamma_{\mathrm{xz}}$ and $\gamma_{\mathrm{yz}}$ are the transverse shear stains introduced through FSDT. Transverse normal rotations and shear strains are transformed in the current formulation into components due to bending and shear as

$$
\begin{aligned}
& \phi_{\mathrm{x}}=\frac{\partial w_{\mathrm{s}}}{\partial x}-\frac{\partial w}{\partial x}=-\frac{\partial w_{\mathrm{b}}}{\partial x} \\
& \phi_{\mathrm{y}}=\frac{\partial w_{\mathrm{s}}}{\partial y}-\frac{\partial w}{\partial y}=-\frac{\partial w_{\mathrm{b}}}{\partial y}
\end{aligned}
$$

\section{Higher-Order Laminate Plate Theory}

In the higher-order shear deformation theory, the first and third components of Kirchhoff's hypothesis are no longer assumed. The displacements then become expressed as a cubic function of the thickness, which allows for a quadratic variation of the transverse shear through the thickness of the ply. This provides a more accurate distribution of transverse 
shear. An illustration comparing the transverse normal deformation of each of the three composite plate theories is shown in Figure 2.3. Reddy's third-order shear deformation

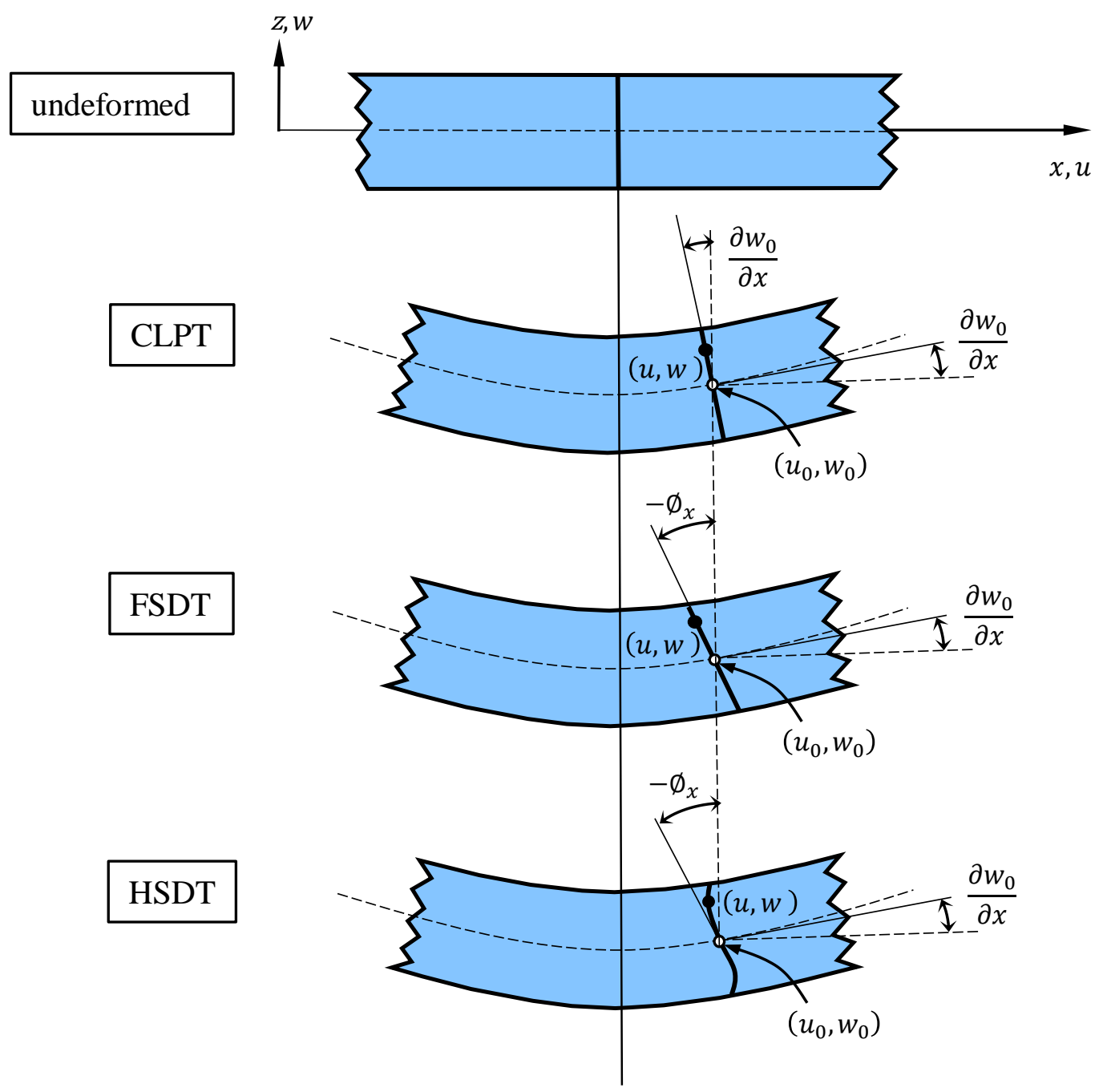

Figure 2.3: Transverse normal deformations for CLPT, FSDT, and HSDT as per Ref. [18]

theory [18] is used, where the displacement field is represented as

$$
\begin{aligned}
& u(x, y, z, t)=u_{0}(x, y, t)+z \phi_{\mathrm{x}}(x, y, z)-z^{3} c_{1}\left(\phi_{\mathrm{x}}+\frac{\partial w_{0}}{\partial x}\right) \\
& v(x, y, z, t)=v_{0}(x, y, t)+z \phi_{\mathrm{y}}(x, y, z)-z^{3} c_{1}\left(\phi_{\mathrm{y}}+\frac{\partial w_{0}}{\partial y}\right) \\
& w(x, y, z, t)=w_{0}(x, y, t)
\end{aligned}
$$

where $c_{1}$ is called a tracer. The FSDT displacement field can be recovered by setting $c_{1}=0$. The tracer value may be found be applying the condition that the transverse shear stress, $\tau_{y z}$ and $\tau_{x z}$, must be zero at the top and bottom surfaces of the laminate 
$(z= \pm h / 2)$. The HSDT tracer is calculated to be

$$
c_{1}=\frac{4}{3 h^{2}}
$$

Using Eq. (2.5), the displacement field may be rewritten as

$$
\begin{aligned}
u(x, y, z, t) & =u_{0}(x, y, t)-z \frac{\partial w_{b}}{\partial x}-c_{1} z^{3} \frac{\partial w_{s}}{\partial x} \\
v(x, y, z, t) & =v_{0}(x, y, t)-z \frac{\partial w_{b}}{\partial y}-c_{1} z^{3} \frac{\partial w_{s}}{\partial y} \\
w(x, y, z, t) & =w_{\mathrm{b}}(x, y, t)+w_{\mathrm{s}}(x, y, t)
\end{aligned}
$$

The nonlinear kinematic strains for moderate rotations are

$$
\begin{gathered}
\left\{\begin{array}{c}
\epsilon_{x} \\
\epsilon_{y} \\
\gamma_{x y}
\end{array}\right\}=\left\{\begin{array}{c}
\epsilon_{x}^{(0)} \\
\epsilon_{y}^{(0)} \\
\gamma_{x y}^{(0)}
\end{array}\right\}+z\left\{\begin{array}{c}
\epsilon_{x}^{(1)} \\
\epsilon_{y}^{(1)} \\
\gamma_{x y}^{(1)}
\end{array}\right\}+z^{3}\left\{\begin{array}{c}
\epsilon_{x}^{(3)} \\
\epsilon_{y}^{(3)} \\
\gamma_{x y}^{(3)}
\end{array}\right\} \\
\left\{\begin{array}{c}
\gamma_{y z} \\
\gamma_{x z}
\end{array}\right\}=\left\{\begin{array}{c}
\gamma_{y z}^{(0)} \\
\gamma_{x z}^{(0)}
\end{array}\right\}+z^{2}\left\{\begin{array}{c}
\gamma_{y z}^{(2)} \\
\gamma_{x z}^{(2)}
\end{array}\right\}
\end{gathered}
$$

The various strain components are

$$
\begin{aligned}
\left\{\epsilon^{(0)}\right\}=\left\{\begin{array}{c}
\epsilon_{x}^{(0)} \\
\epsilon_{y}^{(0)} \\
\gamma_{x y}^{(0)}
\end{array}\right\}=\left\{\begin{array}{c}
\frac{\partial u_{0}}{\partial x}+\frac{1}{2}\left(\frac{\partial w_{b}}{\partial x}\right)^{2}+\frac{1}{2}\left(\frac{\partial w_{s}}{\partial x}\right)^{2} \\
\frac{\partial v_{0}}{\partial y}+\frac{1}{2}\left(\frac{\partial w_{b}}{\partial y}\right)^{2}+\frac{1}{2}\left(\frac{\partial w_{s}}{\partial y}\right)^{2} \\
\frac{\partial u_{0}}{\partial y}+\frac{\partial v_{0}}{\partial x}+\frac{\partial w}{\partial x} \frac{\partial w}{\partial y}
\end{array}\right\} \\
\left\{\epsilon^{(1)}\right\}=\left\{\begin{array}{c}
\epsilon_{x}^{(1)} \\
\epsilon_{y}^{(1)} \\
\gamma_{x y}^{(1)}
\end{array}\right\}=\left\{\begin{array}{c}
-\frac{\partial^{2} w_{b}}{\partial x^{2}} \\
-\frac{\partial^{2} w_{b}}{\partial y^{2}} \\
-2 \frac{\partial^{2} w_{b}}{\partial x \partial y}
\end{array}\right\}
\end{aligned}
$$




$$
\begin{gathered}
\left\{\epsilon^{(3)}\right\}=\left\{\begin{array}{c}
\epsilon_{x}^{(3)} \\
\epsilon_{y}^{(3)} \\
\gamma_{x y}^{(3)}
\end{array}\right\}=c_{1}\left\{\begin{array}{c}
-\frac{\partial^{2} w_{s}}{\partial x^{2}} \\
-\frac{\partial^{2} w_{s}}{\partial y^{2}} \\
-2 \frac{\partial^{2} w_{s}}{\partial x \partial y}
\end{array}\right\} \\
\left\{\gamma^{(0)}\right\}=\left\{\begin{array}{c}
\gamma_{y z}^{(0)} \\
\gamma_{x z}^{(0)}
\end{array}\right\}=\left\{\begin{array}{c}
\frac{\partial w_{s}}{\partial x} \\
\frac{\partial w_{s}}{\partial y}
\end{array}\right\} \\
\left\{\gamma^{(2)}\right\}=\left\{\begin{array}{c}
\gamma_{y z}^{(2)} \\
\gamma_{x z}^{(2)}
\end{array}\right\}=-3 c_{1}\left\{\begin{array}{c}
\frac{\partial w_{s}}{\partial x} \\
\frac{\partial w_{s}}{\partial y}
\end{array}\right\}
\end{gathered}
$$

The non-linear strain component found in the third term of $\gamma_{x y}^{(0)}$ in Eq. (2.11) is neglected.

\subsubsection{Constitutive Equations for HSDT Composite Laminate}

The constitutive relations between stress and strain for an HSDT composite laminate is

$$
\begin{gathered}
\left\{\begin{array}{l}
\{N\} \\
\{M\} \\
\{P\}
\end{array}\right\}=\left[\begin{array}{lll}
{[A]} & {[B]} & {[E]} \\
{[B]} & {[D]} & {[F]} \\
{[E]} & {[F]} & {[H]}
\end{array}\right]\left\{\begin{array}{l}
\left\{\epsilon^{(0)}\right\} \\
\left\{\epsilon^{(1)}\right\} \\
\left\{\epsilon^{(3)}\right\}
\end{array}\right\} \\
\left\{\begin{array}{l}
\{Q\} \\
\{R\}
\end{array}\right\}=\left[\begin{array}{ll}
{[A]} & {[D]} \\
{[D]} & {[F]}
\end{array}\right]\left\{\begin{array}{l}
\left\{\gamma^{(0)}\right\} \\
\left\{\gamma^{(2)}\right\}
\end{array}\right\}
\end{gathered}
$$

where vectors $\{N\}$ and $\{M\}$ are the force and moment resultants, vector $\{Q\}$ is the transverse force resultant, and $\{P\}$ and $\{R\}$ are higher-order stress resultants. The submatrices $[A],[B]$, and $[D]$ are the extension stiffness, bending-extension coupling stiffness, and the bending stiffness, respectively. The force, moment, and higher-order 
stress resultants are

$$
\begin{aligned}
& \left\{\begin{array}{c}
N_{x} \\
N_{y} \\
N_{x y}
\end{array}\right\}=\int_{-\frac{h}{2}}^{\frac{h}{2}}\left\{\begin{array}{c}
\sigma_{x} \\
\sigma_{y} \\
\tau_{x y}
\end{array}\right\} d z \\
& \left\{\begin{array}{l}
M_{x} \\
M_{y} \\
M_{x y}
\end{array}\right\}=\int_{-\frac{h}{2}}^{\frac{h}{2}}\left\{\begin{array}{c}
\sigma_{x} \\
\sigma_{y} \\
\tau_{x y}
\end{array}\right\} z d z \\
& \left\{\begin{array}{c}
P_{x} \\
P_{y} \\
P_{x y}
\end{array}\right\}=\int_{-\frac{h}{2}}^{\frac{h}{2}}\left\{\begin{array}{c}
\sigma_{x} \\
\sigma_{y} \\
\tau_{x y}
\end{array}\right\} z^{3} d z \\
& \left\{\begin{array}{l}
Q_{y} \\
Q_{x}
\end{array}\right\}=\int_{-\frac{h}{2}}^{\frac{h}{2}}\left\{\begin{array}{c}
\tau_{y z} \\
\tau_{x z}
\end{array}\right\} d z \\
& \left\{\begin{array}{l}
R_{y} \\
R_{x}
\end{array}\right\}=\int_{-\frac{h}{2}}^{\frac{h}{2}}\left\{\begin{array}{l}
\tau_{y z} \\
\tau_{x z}
\end{array}\right\} z^{2} d z
\end{aligned}
$$

The stiffness components are defined as

$$
\begin{gathered}
\left(A_{i j}, B_{i j}, D_{i j}, E_{i j}, F_{i j}, H_{i j}\right)=\sum_{k=1}^{n} \int_{z_{k-1}}^{z_{k}} \bar{Q}_{i j}^{(k)}\left(1, z, z^{2}, z^{3}, z^{4}, z^{6}\right) d z \quad i, j=1,2,6 \\
\left(A_{i j}, D_{i j}, F_{i j}\right)=\sum_{k=1}^{n} \int_{z_{k-1}}^{z_{k}} \bar{Q}_{i j}^{(k)}\left(1, z, z^{4}\right) d z \quad i, j=4,5
\end{gathered}
$$


The expanded version of the constitutive relationship (Eq. 2.16) is

$$
\left\{\begin{array}{c}
N_{x} \\
N_{y} \\
N_{x y} \\
M_{x} \\
M_{y} \\
M_{x y} \\
P_{x} \\
P_{y} \\
P_{x y}
\end{array}\right\}=\left[\begin{array}{lllllllll}
A_{11} & A_{12} & A_{16} & B_{11} & B_{12} & B_{16} & E_{11} & E_{12} & E_{16} \\
A_{12} & A_{22} & A_{26} & B_{12} & B_{22} & B_{26} & E_{12} & E_{22} & E_{26} \\
A_{16} & A_{26} & A_{66} & B_{16} & B_{26} & B_{66} & E_{16} & E_{26} & E_{66} \\
B_{11} & B_{12} & B_{16} & D_{11} & D_{12} & D_{16} & F_{11} & F_{12} & F_{16} \\
B_{12} & B_{22} & B_{26} & D_{12} & D_{22} & D_{26} & F_{12} & F_{22} & F_{26} \\
B_{16} & B_{26} & B_{66} & D_{16} & D_{26} & D_{66} & F_{16} & F_{26} & F_{66} \\
E_{11} & E_{12} & E_{16} & F_{11} & F_{12} & F_{16} & H_{11} & H_{12} & H_{16} \\
E_{12} & E_{22} & E_{26} & F_{12} & F_{22} & F_{26} & H_{12} & H_{22} & H_{26} \\
E_{16} & E_{26} & E_{66} & F_{16} & F_{26} & F_{66} & H_{16} & H_{26} & H_{66}
\end{array}\right]\left\{\begin{array}{c}
(0) \\
\epsilon_{x}^{(0)} \\
\epsilon_{y}^{(0)} \\
\gamma_{x y}^{(1)} \\
\epsilon_{x}^{(1)} \\
\epsilon_{y}^{(1)} \\
\gamma_{x y}^{(3)} \\
\epsilon_{x}^{(3)} \\
\gamma_{x y}^{(3)}
\end{array}\right\}
$$

$$
\left\{\begin{array}{c}
Q_{x} \\
R_{y} \\
Q_{x} \\
R_{y}
\end{array}\right\}=\left[\begin{array}{llll}
A_{55} & D_{55} & A_{45} & D_{45} \\
A_{45} & D_{45} & A_{44} & D_{44} \\
D_{55} & F_{55} & D_{45} & F_{45} \\
D_{45} & F_{45} & D_{44} & F_{44}
\end{array}\right]\left\{\begin{array}{c}
\gamma_{x z}^{(0)} \\
\gamma_{y z}^{(0)} \\
\gamma_{x z}^{(2)} \\
\gamma_{y z}^{(2)}
\end{array}\right\}
$$

Eq. (2.26) may be simplified by first reorganizing the $x$ terms first and the $y$ terms thereafter:

$$
\left\{\begin{array}{l}
Q_{x} \\
R_{x} \\
Q_{y} \\
R_{y}
\end{array}\right\}=\left[\begin{array}{cccc}
A_{55} & D_{55} & A_{45} & D_{45} \\
D_{55} & F_{55} & D_{45} & F_{45} \\
A_{45} & D_{45} & A_{44} & D_{44} \\
D_{45} & F_{45} & D_{44} & F_{44}
\end{array}\right]\left\{\begin{array}{c}
\gamma_{x z}^{(0)} \\
\gamma_{x z}^{(2)} \\
\gamma_{y z}^{(0)} \\
\gamma_{y z}^{(2)}
\end{array}\right\}
$$

Now, introduce the following quantities:

$$
\begin{aligned}
D_{i j}^{*} & =A_{i j}-6 c_{1} D_{i j}+9 c_{1}^{2} F_{i j} \quad i, j=4,5 \\
Q_{x}^{*} & =Q_{x}-3 c_{1} R_{x} \\
Q_{y}^{*} & =Q_{y}-3 c_{1} R_{y}
\end{aligned}
$$

A shear correction factor, $K_{s}$, is introduced into Eq. (2.27) despite the fact HSDT does not require one. This is done to obtain FSDT results by letting $c_{1}=0$ and $K_{s}=5 / 6$. 
For HSDT, $K_{s}$ is set equal to one. Eq. (2.27) is simplified as

$$
\left\{\begin{array}{l}
Q_{x}^{*} \\
Q_{y}^{*}
\end{array}\right\}=K_{s}\left[\begin{array}{ll}
D_{44}^{*} & D_{45}^{*} \\
D_{45}^{*} & D_{55}^{*}
\end{array}\right]\left\{\begin{array}{l}
\frac{\partial w_{s}}{\partial y} \\
\frac{\partial w_{s}}{\partial x}
\end{array}\right\}
$$

\subsubsection{Hamilton's Principle}

Hamilton's principle is an application of the principle of virtual displacements to dynamic systems, where the system is characterized by kinetic and potential energy functions. The finite element equations of motion are derived from Hamilton's principle

$$
\int_{t_{1}}^{t_{2}}(\delta U-\delta T-\delta W) d t
$$

where $\delta U$ is the virtual strain energy, $\delta T$ is the virtual kinetic energy, and $\delta W$ is the virtual work.

\subsubsection{Virtual Strain Energy}

The virtual strain energy is used to formulate the finite element stiffness matrix. The expression for the virtual strain energy, $\delta U$, over the plate is given by

$$
\delta U=\iiint_{V}\left(\sigma_{x} \delta \epsilon_{x}+\sigma_{y} \delta \epsilon_{y}+\tau_{x y} \delta \gamma_{x y}+\tau_{x z} \delta \gamma_{x z}+\tau_{y z} \delta \gamma_{y z}\right) d V
$$

where $\delta \epsilon_{x}, \delta \epsilon_{y}, \delta \gamma_{x y}, \delta \gamma_{x z}$, and $\delta \gamma_{y z}$ are the virtual strains and $V$ is the volume of the plate. The volume integral is separated into an integral over the plate area in the $x, y$ directions and an integral over the thickness coordinate, $z$.

$$
\delta U=\iint_{A} \int_{-\frac{h}{2}}^{\frac{h}{2}}\left[\sigma_{x} \delta \epsilon_{x}+\sigma_{y} \delta \epsilon_{y}+\tau_{x y} \delta \gamma_{x y}+\tau_{x z} \delta \gamma_{x z}+\tau_{y z} \delta \gamma_{y z}\right] d A d z
$$

Substituting for the force, moment, and stress resultants from Eqs. (2.18 - 2.22) and the strain subcomponents, 


$$
\begin{aligned}
\delta U=\iint_{A} & {\left[N_{x} \delta \epsilon_{x}^{(0)}+M_{x} \delta \epsilon_{x}^{(1)}+P_{x} \delta \epsilon_{x}^{(3)}+N_{y} \delta \epsilon_{y}^{(0)}+M_{y} \delta \epsilon_{y}^{(1)}+P_{y} \delta \epsilon_{y}^{(3)}+N_{x y} \delta \gamma_{x y}^{(0)}\right.} \\
+ & \left.M_{x y} \delta \gamma_{x y}^{(1)}+P_{x y} \delta \gamma_{x y}^{(3)}+Q_{x} \delta \gamma_{x z}^{(0)}+R_{x} \delta \gamma_{x z}^{(2)}+Q_{y} \delta \gamma_{y z}^{(0)}+R_{y} \delta \gamma_{y z}^{(2)}\right] d A
\end{aligned}
$$

A shorthand notation is introduced to describe differentiated variables as

$$
\begin{aligned}
& ()^{x}=\frac{\partial()}{\partial x} \\
& ()^{y}=\frac{\partial()}{\partial y} \\
& \left.()^{\cdot}\right)=\frac{\partial()}{\partial t}
\end{aligned}
$$

Finally, the virtual strain energy is listed with the strain components fully written out using the shorthand notation.

$$
\begin{aligned}
\delta U=\iint_{A}[ & N_{x}\left(\delta u_{0}^{x}+w_{b}^{x} \delta w_{b}^{x}+w_{s}^{x} \delta w_{s}^{x}\right)-M_{x} \delta w_{b}^{x x}-P_{x} c_{1} \delta w_{s}^{x x}+N_{y}\left(\delta v_{0}^{y}+w_{b}^{y} \delta w_{b}^{y}+\right. \\
& \left.w_{s}^{y} \delta w_{s}^{y}\right)-M_{y} \delta w_{b}^{y y}-P_{y} c_{1} \delta w_{s}^{y y}+N_{x y}\left(\delta u_{0}^{y}+\delta v_{0}^{x}\right)-2 M_{x y} \delta w_{b}^{x y}- \\
& \left.2 P_{x y} c_{1} \delta w_{s}^{x y}+Q_{x}^{*} \delta w_{s}^{x}+Q_{y}^{*} \delta w_{s}^{y}\right] d A
\end{aligned}
$$

\subsubsection{Virtual Kinetic Energy}

The virtual kinetic energy is used to formulate the finite element inertia matrix. The expression for the plate virtual kinetic energy, $\delta T$, is given by

$$
\delta T=\iiint_{V} \rho(\dot{u} \delta \dot{u}+\dot{v} \delta \dot{v}+\dot{w} \delta \dot{w}) d V
$$

where $\rho$ is the material density. This expression is expanded using the displacement field from Eq. (2.8)

$$
\begin{gathered}
\delta T=\iiint_{V} \rho\left[\left(\dot{u}_{0}-z \dot{w}_{b}^{x}-c_{1} z^{3} \dot{w}_{s}^{x}\right)\left(\delta \dot{u}_{0}-z \delta \dot{w}_{b}^{x}-c_{1} z^{3} \delta \dot{w}_{s}^{x}\right)+\left(\dot{v}_{0}-z \dot{w}_{b}^{y}-c_{1} z^{3} \dot{w}_{s}^{y}\right)\right. \\
\left.\left(\delta \dot{v}_{0}-z \delta \dot{w}_{b}^{y}-c_{1} z^{3} \delta \dot{w}_{s}^{y}\right)+\left(\dot{w}_{b}+\dot{w}_{s}\right)\left(\delta \dot{w}_{b}+\delta \dot{w}_{s}\right)\right] d V
\end{gathered}
$$


Rearranging the expanded expression,

$$
\begin{aligned}
\delta T=\iiint_{V} \rho & {\left[\left(\dot{u}_{0}-z \dot{w}_{b}^{x}-c_{1} z^{3} \dot{w}_{s}^{x}\right) \delta \dot{u}_{0}-\left(z \dot{u}_{0}-z^{2} \dot{w}_{b}^{x}-c_{1} z^{4} \dot{w}_{s}^{x}\right) \delta \dot{w}_{b}^{x}-c_{1}\left(z^{3} \dot{u}_{0}-z^{4} \dot{w}_{b}^{x}\right.\right.} \\
& \left.-c_{1} z^{6} \dot{w}_{s}^{x}\right) \delta \dot{w}_{s}^{x}+\left(\dot{v}_{0}-z \dot{w}_{b}^{y}-c_{1} z^{3} \dot{w}_{s}^{y}\right) \delta \dot{v}_{0}-\left(z \dot{v}_{0}-z^{2} \dot{w}_{b}^{y}-c_{1} z^{4} \dot{w}_{s}^{y}\right) \delta \dot{w}_{b}^{y}- \\
& \left.c_{1}\left(z^{3} \dot{v}_{0}-z^{4} \dot{w}_{b}^{y}-c_{1} z^{6} \dot{w}_{s}^{y}\right) \delta \dot{w}_{s}^{y}+\left(\dot{w}_{b}+\dot{w}_{s}\right) \delta \dot{w}_{b}+\left(\dot{w}_{b}+\dot{w}_{s}\right) \delta \dot{w}_{s}\right] d V
\end{aligned}
$$

Considering the time integral from Hamilton's principle [Eq. (2.30)],

$$
\begin{aligned}
-\int_{t_{1}}^{t_{2}} \delta T d t= & \iiint_{V}\left\{\int_{t_{1}}^{t_{2}}-\rho\left[\left(\dot{u}_{0}-z \dot{w}_{b}^{x}-c_{1} z^{3} \dot{w}_{s}^{x}\right) \delta \dot{u}_{0}-\left(z \dot{u}_{0}-z^{2} \dot{w}_{b}^{x}-c_{1} z^{4} \dot{w}_{s}^{x}\right) \delta \dot{w}_{b}^{x}\right.\right. \\
& -c_{1}\left(z^{3} \dot{u}_{0}-z^{4} \dot{w}_{b}^{x}-c_{1} z^{6} \dot{w}_{s}^{x}\right) \delta \dot{w}_{s}^{x}+\left(\dot{v}_{0}-z \dot{w}_{b}^{y}-c_{1} z^{3} \dot{w}_{s}^{y}\right) \delta \dot{v}_{0}-\left(z \dot{v}_{0}-\right. \\
& \left.z^{2} \dot{w}_{b}^{y}-c_{1} z^{4} \dot{w}_{s}^{y}\right) \delta \dot{w}_{b}^{y}-c_{1}\left(z^{3} \dot{v}_{0}-z^{4} \dot{w}_{b}^{y}-c_{1} z^{6} \dot{w}_{s}^{y}\right) \delta \dot{w}_{s}^{y}+\left(\dot{w}_{b}+\dot{w}_{s}\right) \delta \dot{w}_{b} \\
& \left.\left.+\left(\dot{w}_{b}+\dot{w}_{s}\right) \delta \dot{w}_{s}\right] d t\right\} d V
\end{aligned}
$$

The time integral is integrated by parts to eliminate terms containing time derivatives of virtual displacements and the boundary terms are grouped as

$$
\iiint_{V}[B(t)]_{t_{1}}^{t_{2}} d V
$$

This leads to the updated expression

$$
\begin{aligned}
-\int_{t_{1}}^{t_{2}} \delta T d t= & \iiint_{V}\left\{\int _ { t _ { 1 } } ^ { t _ { 2 } } \rho \left[\left(\ddot{u}_{0}-z \ddot{w}_{b}^{x}-c_{1} z^{3} \ddot{w}_{s}^{x}\right) \delta u_{0}-\left(z \ddot{u}_{0}-z^{2} \ddot{w}_{b}^{x}-c_{1} z^{4} \ddot{w}_{s}^{x}\right) \delta w_{b}^{x}\right.\right. \\
& -c_{1}\left(z^{3} \ddot{u}_{0}-z^{4} \ddot{w}_{b}^{x}-c_{1} z^{6} \ddot{w}_{s}^{x}\right) \delta w_{s}^{x}+\left(\ddot{v}_{0}-z \ddot{w}_{b}^{y}-c_{1} z^{3} \ddot{w}_{s}^{y}\right) \delta v_{0}-\left(z \ddot{v}_{0}-\right. \\
& \left.z^{2} \ddot{w}_{b}^{y}-c_{1} z^{4} \ddot{w}_{s}^{y}\right) \delta w_{b}^{y}-c_{1}\left(z^{3} \ddot{v}_{0}-z^{4} \ddot{w}_{b}^{y}-c_{1} z^{6} \ddot{w}_{s}^{y}\right) \delta w_{s}^{y}+\left(\ddot{w}_{b}+\ddot{w}_{s}\right) \delta w_{b} \\
& \left.\left.+\left(\ddot{w}_{b}+\ddot{w}_{s}\right) \delta w_{s}\right] d t+[B(t)]_{t_{1}}^{t_{2}}\right\} d V
\end{aligned}
$$

The virtual displacements no longer explicitly include time derivatives such that the integrand is valid for all time and may be removed from the time integral. Additionally, the boundary terms, $B(t)$, may be neglected because they do not contribute to the finite element inertia matrix. The volume integral is split into an integral over the plate area 
and another across the thickness. Mass moment of inertia terms are introduced as

$$
I_{k}=\int_{-\frac{h}{2}}^{\frac{h}{2}} \rho z^{k} d z \quad k=0,1,2, \ldots, 6
$$

where $I_{0}, I_{1}$, and $I_{2}$, are the normal, coupled normal-rotary, and rotary inertia terms, respectively, and those remaining are higher-order terms. Substituting the mass moments of inertia, the final virtual kinetic energy expression becomes

$$
\begin{aligned}
-\delta T= & \iint_{A}\left[\left(I_{0} \ddot{u}_{0}-I_{1} \ddot{w}_{b}^{x}-c_{1} I_{3} \ddot{w}_{s}^{x}\right) \delta u_{0}-\left(I_{1} \ddot{u}_{0}-I_{2} \ddot{w}_{b}^{x}-c_{1} I_{4} \ddot{w}_{s}^{x}\right) \delta w_{b}^{x}\right. \\
& -c_{1}\left(I_{3} \ddot{u}_{0}-I_{4} \ddot{w}_{b}^{x}-c_{1} I_{6} \ddot{w}_{s}^{x}\right) \delta w_{s}^{x}+\left(I_{0} \ddot{v}_{0}-I_{1} \ddot{w}_{b}^{y}-c_{1} I_{3} \ddot{w}_{s}^{y}\right) \delta v_{0} \\
& -\left(I_{1} \ddot{v}_{0}-I_{2} \ddot{w}_{b}^{y}-c_{1} I_{4} \ddot{w}_{s}^{y}\right) \delta w_{b}^{y}-c_{1}\left(I_{3} \ddot{v}_{0}-I_{4} \ddot{w}_{b}^{y}-c_{1} I_{6} \ddot{w}_{s}^{y}\right) \delta w_{s}^{y} \\
& \left.+I_{0}\left(\ddot{w}_{b}+\ddot{w}_{s}\right) \delta w_{b}+I_{0}\left(\ddot{w}_{b}+\ddot{w}_{s}\right) \delta w_{s}\right] d x d y
\end{aligned}
$$




\subsection{Finite Element Formulation}

\subsubsection{Introduction}

The finite element method is a powerful numerical procedure to solve various types of engineering problems. Engineering systems are often complex and governed by partial differential equations with exact solutions that are difficult or impossible to solve. The finite element method circumnavigates this difficulty by discretizing these systems into a mesh of finite elements, which are related though algebraic equations and solved numerically for an approximate solution. In structural analysis, the method is formulated based on the principles of conservation of energy and variational calculus, where the approximate solution is obtained by minimizing a potential energy function. The formulation of finite element plates are inherently more complicated than that of beams because they are two-dimensional. In this section, fundamental finite element plate concepts are first explored before a detailed formulation of the current element.

\section{Interpolation Functions, Continuity, and Conformity}

An important component of the finite element plate formulation is determining the interpolation functions used to approximate the displacements. When a variable has first-order derivatives of displacements in the virtual stain and kinetic energy, the variable requires only $C^{0}$ continuity, where interelement displacements are continuous but slopes are not. These variables are approximated with Lagrange interpolation functions. When a variable has second-order derivatives in the virtual stain and kinetic energy, the variable requires $C^{1}$ continuity, where both the interelement displacements and slopes are continuous. These variables should be approximated with Hermite interpolation functions [18]. Whether a plate theory requires $C^{1}$ continuity or not has major implications on its accuracy and capacity for transformation.

There are two kinds of $C^{1}$ continuous plate elements. A conforming element is one where the continuity of the lateral displacement, $w$, and the normal slope, $\partial w / \partial n$, are truly satisfied between elements. This is done by ensuring both $w$ and $\partial w / \partial n$ are uniquely defined along each element boundary. To achieve a unique variation of $\partial w / \partial n$ along a given boundary, there must be at least the same number of DOF available to define $\partial w / \partial n$ as the order of its variation. For example, if $\partial w / \partial n$ varies cubically along a boundary, there must be three DOF available along that boundary to uniquely define its cubic variation. A non-conforming element is one where the the continuity of $\partial w / \partial n$ is not satisfied between elements. Non-conforming elements have an inadequate number of DOF capable of uniquely defining $\partial w / \partial n$ along a boundary and can have difficulty with convergence [18], [17]. Thus, it is possible to attempt to achieve genuine $C^{1}$ continuity using Hermite shape functions but still fail due to non-conformity. 


\subsubsection{MONNA Plate Element}

The goal of this section is to formulate a new, conforming Multi-Order Nine-Node Advanced (MONNA) composite plate element based on the HSDT as shown in Figure 2.4. This element was conceptually invented by Sivaneri [19] and developed by the present author.

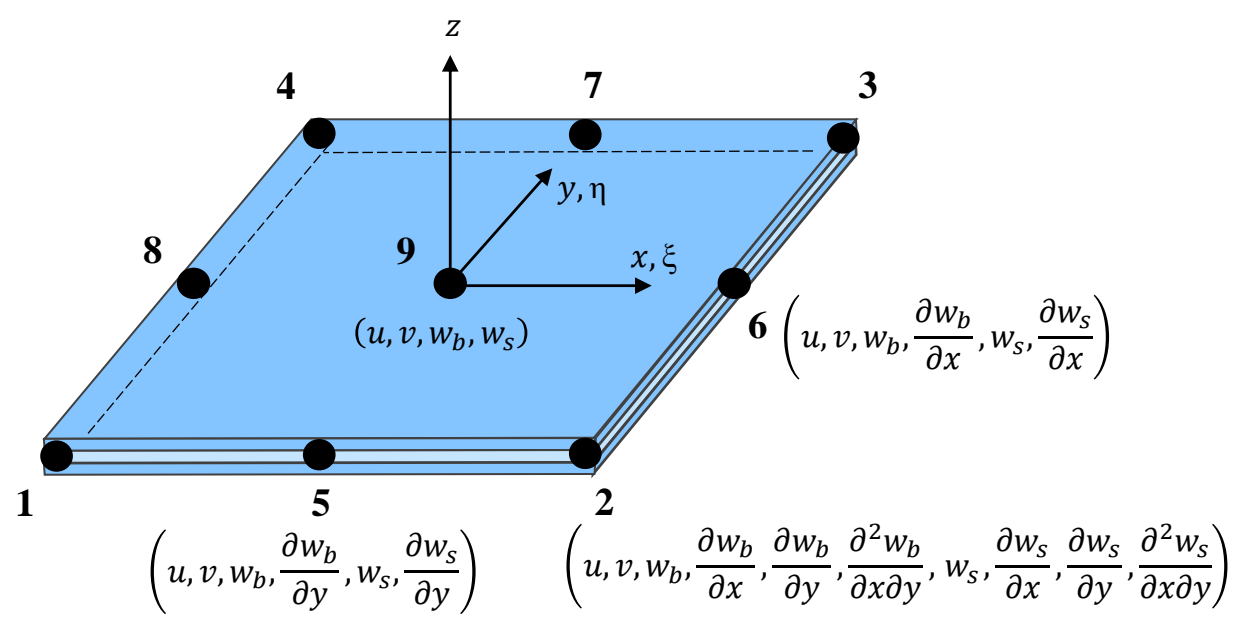

Figure 2.4: MONNA plate element with physical DOF distribution

This is a subparametric master element with a plate coordinate system of $(\xi, \eta, z)$. The dependent variables include in-plane displacements in the $x$ and $y$ directions, $u$ and $v$, and the lateral displacements, $w_{b}$ and $w_{s}$. There are four corner nodes (1-4), four midside nodes (5-8), and a ninth node in the center. In total, there are 68 degrees of freedom. This element may be considered $h-p$ version, as accuracy is increased by increasing the number of elements and internal nodes are included to aid the rate of convergence. The coordinate transformation for a general plate element is first explored.

\subsubsection{Coordinate Transformation}

It's important for the rectangular plate element to have the capacity for quadrilateral transformation such that realistic aeroelastic wing structure may be modeled. An arbitrary quadrilateral element in the physical domain is shown in Figure 2.5, where the coordinates of the four corners are defined. The goal is to transform the geometry between the physical domain $(x, y)$ to a square in the standard domain $(\xi, \eta)$ and vice versa. Bilinear shape functions corresponding to a four-node isoparametric plane element are used for this purpose, which are adequate for transforming

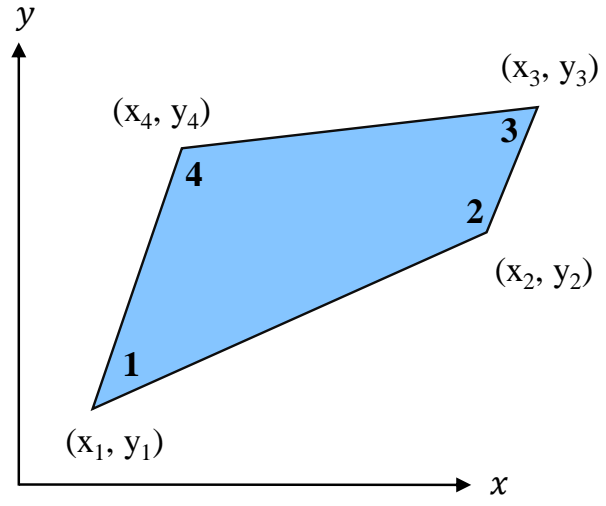

Figure 2.5: Physical quadrilateral 
straight edges.

The coordinate transformation between the standard and physical domain is related through the following four bilinear shape functions

$$
\begin{array}{r}
x(\xi, \eta)=\left\lfloor\begin{array}{llll}
N_{1} & N_{2} & N_{3} & N_{4}
\end{array}\right\rfloor\left\{\begin{array}{l}
x_{1} \\
x_{2} \\
x_{3} \\
x_{4}
\end{array}\right\} \\
y(\xi, \eta)=\left\lfloor\begin{array}{llll}
N_{1} & N_{2} & N_{3} & N_{4}
\end{array}\right\rfloor\left\{\begin{array}{l}
y_{1} \\
y_{2} \\
y_{3} \\
y_{4}
\end{array}\right\}
\end{array}
$$

The shape functions are specified as

$$
\begin{aligned}
N_{1} & =\frac{1}{4}(1-\xi)(1-\eta) \\
N_{2} & =\frac{1}{4}(1+\xi)(1-\eta) \\
N_{3} & =\frac{1}{4}(1+\xi)(1+\eta) \\
N_{4} & =\frac{1}{4}(1-\xi)(1+\eta)
\end{aligned}
$$

\section{First Derivatives}

The first derivatives in the standard domain are related to those in the physical through the following two expressions

$$
\begin{gathered}
\frac{\partial}{\partial \xi}=\frac{\partial}{\partial x} \frac{\partial x}{\partial \xi}+\frac{\partial}{\partial y} \frac{\partial y}{\partial \xi} \\
\frac{\partial}{\partial \eta}=\frac{\partial}{\partial x} \frac{\partial x}{\partial \eta}+\frac{\partial}{\partial y} \frac{\partial y}{\partial \eta}
\end{gathered}
$$

These are organized into matrix notation,

$$
\left\{\begin{array}{l}
\frac{\partial}{\partial \xi} \\
\frac{\partial}{\partial \eta}
\end{array}\right\}=\left[\begin{array}{ll}
\frac{\partial x}{\partial \xi} & \frac{\partial y}{\partial \xi} \\
\frac{\partial x}{\partial \eta} & \frac{\partial y}{\partial \eta}
\end{array}\right]\left\{\begin{array}{c}
\frac{\partial}{\partial x} \\
\frac{\partial}{\partial y}
\end{array}\right\}
$$

The square matrix in Eq. (2.47) is the Jacobian matrix and denoted as $[J]$ 


$$
[J]=\left[\begin{array}{ll}
J_{11} & J_{12} \\
J_{21} & J_{22}
\end{array}\right]
$$

The Jacobian matrix terms may be specified as

$$
\begin{aligned}
& J_{11}=\frac{\partial x}{\partial \xi}=\frac{1}{4}\left[-(1-\eta) x_{1}+(1-\eta) x_{2}+(1+\eta) x_{3}-(1+\eta) x_{4}\right] \\
& J_{21}=\frac{\partial x}{\partial \eta}=\frac{1}{4}\left[-(1-\xi) x_{1}-(1+\xi) x_{2}+(1+\xi) x_{3}+(1-\xi) x_{4}\right] \\
& J_{12}=\frac{\partial y}{\partial \xi}=\frac{1}{4}\left[-(1-\eta) y_{1}+(1-\eta) y_{2}+(1+\eta) y_{3}-(1+\eta) y_{4}\right] \\
& J_{22}=\frac{\partial y}{\partial \eta}=\frac{1}{4}\left[-(1-\xi) y_{1}-(1+\xi) y_{2}+(1+\xi) y_{3}+(1-\xi) y_{4}\right]
\end{aligned}
$$

The determinant of the Jacobian matrix is simply called the Jacobian,

$$
|J|=\left|\begin{array}{ll}
J_{11} & J_{12} \\
J_{21} & J_{22}
\end{array}\right|
$$

The Jacobian is an important parameter that relates integration in the standard domain to the physical. This transformation is useful because it is much easier to carry out numerical integration in the standard domain. The equivalence is

$$
\iint_{A} F(x, y) d x d y=\int_{-1}^{1} \int_{-1}^{1} F[x(\xi, \eta), y(\xi, \eta)]|J| d \xi d \eta
$$

\section{Second Derivatives}

The second derivatives are found by simply differentiating the first derivatives. The second derivative with respect to $\xi$ becomes

$$
\begin{aligned}
\frac{\partial^{2}}{\partial \xi^{2}} & =\frac{\partial}{\partial \xi}\left(\frac{\partial}{\partial x} \frac{\partial x}{\partial \xi}+\frac{\partial}{\partial y} \frac{\partial y}{\partial \xi}\right)=\left(\frac{\partial}{\partial x} \frac{\partial x}{\partial \xi}+\frac{\partial}{\partial y} \frac{\partial y}{\partial \xi}\right)\left(\frac{\partial}{\partial x} \frac{\partial x}{\partial \xi}+\frac{\partial}{\partial y} \frac{\partial y}{\partial \xi}\right) \\
& =\frac{\partial^{2}}{\partial x^{2}}\left(\frac{\partial x}{\partial \xi}\right)^{2}+\frac{\partial^{2}}{\partial x \partial y} \frac{\partial x}{\partial \xi} \frac{\partial y}{\partial \xi}+\frac{\partial}{\partial x} \frac{\partial^{2} x}{\partial \xi^{2}}+\frac{\partial^{2}}{\partial x \partial y} \frac{\partial x}{\partial \xi} \frac{\partial y}{\partial \xi}+\frac{\partial^{2}}{\partial y^{2}}\left(\frac{\partial y}{\partial \xi}\right)^{2}+\frac{\partial}{\partial y} \frac{\partial^{2} y}{\partial \xi^{2}}
\end{aligned}
$$

Substituting the Jacobian terms, the expression becomes

$$
\frac{\partial^{2}}{\partial \xi^{2}}=\frac{\partial J_{11}}{\partial \xi} \frac{\partial}{\partial x}+\frac{\partial J_{12}}{\partial \xi} \frac{\partial}{\partial y}+J_{11}^{2} \frac{\partial^{2}}{\partial x^{2}}+J_{12}^{2} \frac{\partial^{2}}{\partial y^{2}}+2 J_{11} J_{12} \frac{\partial^{2}}{\partial x \partial y}
$$


Similarly, the second derivative with respect to $\eta$ is

$$
\frac{\partial^{2}}{\partial \eta^{2}}=\frac{\partial J_{21}}{\partial \eta} \frac{\partial}{\partial x}+\frac{\partial J_{22}}{\partial \eta} \frac{\partial}{\partial y}+J_{21}^{2} \frac{\partial^{2}}{\partial x^{2}}+J_{22}^{2} \frac{\partial^{2}}{\partial y^{2}}+2 J_{21} J_{22} \frac{\partial^{2}}{\partial x \partial y}
$$

The cross derivative with respect to $\xi$ and $\eta$ is

$$
\frac{\partial^{2}}{\partial \xi \partial \eta}=\frac{\partial J_{11}}{\partial \eta} \frac{\partial}{\partial x}+\frac{\partial J_{12}}{\partial \eta} \frac{\partial}{\partial y}+J_{11} J_{21} \frac{\partial^{2}}{\partial x^{2}}+J_{12} J_{22} \frac{\partial^{2}}{\partial y^{2}}+\left(J_{11} J_{22}+J_{12} J_{21}\right) \frac{\partial^{2}}{\partial x \partial y}
$$

The second derivatives are organized in matrix notation for a four corner quadrilateral. Note that $J_{11}^{\xi}, J_{12}^{\xi}, J_{21}^{\eta}$, and $J_{22}^{\eta}$ are zero in this case.

$$
\left\{\begin{array}{c}
\frac{\partial^{2}}{\partial \xi^{2}} \\
\frac{\partial^{2}}{\partial \eta^{2}} \\
\frac{\partial^{2}}{\partial \xi \partial \eta}
\end{array}\right\}=\left[\begin{array}{cc}
0 & 0 \\
0 & 0 \\
J_{11}^{\eta} & J_{12}^{\eta}
\end{array}\right]\left\{\begin{array}{c}
\frac{\partial}{\partial x} \\
\frac{\partial}{\partial y}
\end{array}\right\}+\left[\begin{array}{ccc}
J_{11}^{2} & J_{12}^{2} & 2 J_{11} J_{12} \\
J_{21}^{2} & J_{22}^{2} & 2 J_{21} J_{22} \\
J_{11} J_{21} & J_{12} J_{22} & J_{11} J_{22}+J_{12} J_{21}
\end{array}\right]\left\{\begin{array}{c}
\frac{\partial^{2}}{\partial x^{2}} \\
\frac{\partial^{2}}{\partial y^{2}} \\
\frac{\partial^{2}}{\partial x \partial y}
\end{array}\right\}
$$

\section{Inverse Transformation}

The transformation from the standard domain to the physical is also needed. The inverse of the Jacobian matrix, $\left[J^{*}\right]$, is

$$
\left[J^{*}\right]=\left[\begin{array}{cc}
J_{11} & J_{12} \\
J_{21} & J_{22}
\end{array}\right]^{-1}=\left[\begin{array}{cc}
J_{11}^{*} & J_{12}^{*} \\
J_{21}^{*} & J_{22}^{*}
\end{array}\right]
$$

The inverse first derivative relationship becomes

$$
\left\{\begin{array}{l}
\frac{\partial}{\partial x} \\
\frac{\partial}{\partial y}
\end{array}\right\}=\left[\begin{array}{cc}
J_{11}^{*} & J_{12}^{*} \\
J_{21}^{*} & J_{22}^{*}
\end{array}\right]\left\{\begin{array}{c}
\frac{\partial}{\partial \xi} \\
\frac{\partial}{\partial \eta}
\end{array}\right\}
$$

The inverse second derivatives are given by

$$
\left\{\begin{array}{c}
\frac{\partial^{2}}{\partial x^{2}} \\
\frac{\partial^{2}}{\partial y^{2}} \\
\frac{\partial^{2}}{\partial x \partial y}
\end{array}\right\}=\left[\begin{array}{ll}
\left(T_{A}\right)_{11} & \left(T_{A}\right)_{12} \\
\left(T_{A}\right)_{21} & \left(T_{A}\right)_{22} \\
\left(T_{A}\right)_{31} & \left(T_{A}\right)_{32}
\end{array}\right]\left\{\begin{array}{c}
\frac{\partial}{\partial \xi} \\
\frac{\partial}{\partial \eta}
\end{array}\right\}+\left[\begin{array}{ccc}
\left(J_{11}^{*}\right)^{2} & \left(J_{12}^{*}\right)^{2} & 2 J_{11}^{*} J_{12}^{*} \\
\left(J_{21}^{*}\right)^{2} & \left(J_{22}^{*}\right)^{2} & 2 J_{21}^{*} J_{22}^{*} \\
J_{11}^{*} J_{21}^{*} & J_{12}^{*} J_{22}^{*} & J_{11}^{*} J_{22}^{*}+J_{12}^{*} J_{21}^{*}
\end{array}\right]\left\{\begin{array}{c}
\frac{\partial^{2}}{\partial \xi^{2}} \\
\frac{\partial^{2}}{\partial \eta^{2}} \\
\frac{\partial^{2}}{\partial \xi \partial \eta}
\end{array}\right\}
$$


where for a four corner quadrilateral, the $T_{A}$ transformation matrix may be shown to be

$$
T_{A}=\left[\begin{array}{cc}
2 J_{11}^{*} J_{12}^{*} J_{11}^{\eta} & 2 J_{11}^{*} J_{12}^{*} J_{12}^{\eta} \\
2 J_{21}^{*} J_{22}^{*} J_{11}^{\eta} & 2 J_{21}^{*} J_{22}^{*} J_{12}^{\eta} \\
\left(J_{11}^{*} J_{22}^{*}+J_{12}^{*} J_{21}^{*}\right) J_{11}^{\eta} & \left(J_{11}^{*} J_{22}^{*}+J_{12}^{*} J_{21}^{*}\right) J_{12}^{\eta}
\end{array}\right]\left[\begin{array}{cc}
J_{11}^{*} & J_{12}^{*} \\
J_{21}^{*} & J_{22}^{*}
\end{array}\right]
$$

\subsubsection{Shape Functions}

The finite element plate shape functions are derived in the standard domain. Referencing the composite plate virtual strain and kinetic energy expressions from Eqs. (2.35) and (2.43), the in-plane displacements, $u$ and $v$, have only first-order derivatives such that only $C^{0}$ continuity is needed and Lagrange interpolation may be employed. The lateral displacements, $w_{b}$ and $w_{s}$ have second-order derivatives, which requires $C^{1}$ continuity and Hermite interpolation. These conclusions are in agreement with the established knowledge that $C^{1}$ continuity is a requirement for Reddy's higher-order shear deformation theory [15]. It's important to note that the more widely used shear deformation theory, FSDT, only requires $C^{0}$ continuity when the lateral displacement DOF remain as $w, \phi_{x}$, and $\phi_{y}$.

\section{Lagrange Interpolation}

Quadratic Lagrange shape functions for the elements' nine-node arrangement are used to approximate the in-plane displacements. They are listed as follows [18]:

$$
\begin{aligned}
& H_{L 1}(\xi, \eta)=\frac{\xi \eta}{4}(1-\xi)(1-\eta) \\
& H_{L 2}(\xi, \eta)=-\frac{\xi \eta}{4}(1+\xi)(1-\eta) \\
& H_{L 3}(\xi, \eta)=\frac{\xi \eta}{4}(1+\xi)(1+\eta) \\
& H_{L 4}(\xi, \eta)=-\frac{\xi \eta}{4}(1-\xi)(1+\eta) \\
& H_{L 5}(\xi, \eta)=-\frac{\eta}{2}\left(1-\xi^{2}\right)(1-\eta) \\
& H_{L 6}(\xi, \eta)=\frac{\xi}{2}(1+\xi)\left(1-\eta^{2}\right) \\
& H_{L 7}(\xi, \eta)=\frac{\eta}{2}\left(1-\xi^{2}\right)(1+\eta) \\
& H_{L 8}(\xi, \eta)=-\frac{\xi}{2}(1-\xi)\left(1-\eta^{2}\right) \\
& H_{L 9}(\xi, \eta)=\left(1-\xi^{2}\right)\left(1-\eta^{2}\right)
\end{aligned}
$$

The set of Lagrange shape functions is 


$$
\left\lfloor H_{L}(\xi, \eta)\right\rfloor=\left\lfloor\begin{array}{llll}
H_{L 1} & H_{L 2} & \ldots & H_{L 9}
\end{array}\right\rfloor
$$

The $u$ in-plane displacement, for example, is given by

$$
u(\xi, \eta)=\left\lfloor H_{L}(\xi, \eta)\right\rfloor\left\{q_{u}\right\}
$$

where $\left\{q_{u}\right\}$ is the axial displacement element degree of freedom vector for the MONNA element.

\section{Hermite Interpolation}

Hermite shape functions for this nine-node arrangement, to the best of the author's knowledge, are not present in literature such that they must be derived. The MONNA plate element has the following distribution of bending lateral displacement DOF in the standard domain. The shear lateral displacement, $w_{s}$, has the same distribution.

$$
\begin{gathered}
\text { Corner nodes } 1,2,3,4: w_{b}, \frac{\partial w_{b}}{\partial \xi}, \frac{\partial w_{b}}{\partial \eta}, \frac{\partial^{2} w_{b}}{\partial \xi \partial \eta} \\
\text { Midside nodes } 5,7: w_{b}, \frac{\partial w_{b}}{\partial \eta} \\
\text { Midside nodes } 6,8: w_{b}, \frac{\partial w_{b}}{\partial \xi}
\end{gathered}
$$

Center node $9: w_{b}$

Thus, there are 25 degrees of freedom associated with lateral displacement. This displacement within the element is assumed to have the form:

$$
w_{b}(\xi, \eta)=\sum_{i=0}^{4} \sum_{j=0}^{4} a_{i j} \xi^{i} \eta^{j}
$$

This expression is expanded with coefficients in single-subscript notation,

$$
\begin{aligned}
w_{b}(\xi, \eta)= & a_{0}+a_{1} \xi+a_{2} \eta+a_{3} \xi^{2}+a_{4} \xi \eta+a_{5} \eta^{2}+a_{6} \xi^{3}+a_{7} \xi^{2} \eta+a_{8} \xi \eta^{2}+a_{9} \eta^{3}+a_{10} \xi^{4}+ \\
& a_{11} \xi^{3} \eta+a_{12} \xi^{2} \eta^{2}+a_{13} \xi \eta^{3}+a_{14} \eta^{4}+a_{15} \xi^{4} \eta+a_{16} \xi^{3} \eta^{2}+a_{17} \xi^{2} \eta 3+a_{18} \xi \eta^{4}+ \\
& a_{19} \xi^{4} \eta^{2}+a_{20} \xi^{3} \eta^{3}+a_{21} \xi^{2} \eta^{4}+a_{22} \xi^{4} \eta^{3}+a_{23} \xi^{3} \eta^{4}+a_{24} \xi^{4} \eta^{4}
\end{aligned}
$$

The standard procedure of substituting nodal coordinates into the degrees of freedom in the form of Eq. (2.66), solving for the unknown coefficients, $a_{i j}$, and obtaining the shape 
functions is performed. These resulting shape functions are the tensor products of the quartic Hermite polynomials in $\xi$ and $\eta$. The 25 Hermite shape functions are as follows:

$$
\begin{aligned}
& \bar{H}_{1}=\frac{9}{16} \xi \eta-\frac{3}{4} \xi \eta^{2}-\frac{3}{16} \xi \eta^{3}+\frac{3}{8} \xi \eta^{4}-\frac{3}{4} \xi^{2} \eta+\xi^{2} \eta^{2}+\frac{1}{4} \xi^{2} \eta^{3}-\frac{1}{2} \xi^{2} \eta^{4}-\frac{3}{16} \xi^{3} \eta+\frac{1}{4} \xi^{3} \eta^{2}+ \\
& \frac{1}{16} \xi^{3} \eta^{3}-\frac{1}{8} \xi^{3} \eta^{4}+\frac{3}{8} \xi^{4} \eta-\frac{1}{2} \xi^{4} \eta^{2}-\frac{1}{8} \xi^{4} \eta^{3}+\frac{1}{4} \xi^{4} \eta^{4} \\
& \bar{H}_{2}=\frac{3}{16} \xi \eta-\frac{1}{4} \xi \eta^{2}-\frac{1}{16} \xi \eta^{3}+\frac{1}{8} \xi \eta^{4}-\frac{3}{16} \xi^{2} \eta+\frac{1}{4} \xi^{2} \eta^{2}+\frac{1}{16} \xi^{2} \eta^{3}-\frac{1}{8} \xi^{2} \eta^{4}-\frac{3}{16} \xi^{3} \eta+ \\
& \frac{1}{4} \xi^{3} \eta^{2}+\frac{1}{16} \xi^{3} \eta^{3}-\frac{1}{8} \xi^{3} \eta^{4}+\frac{3}{16} \xi^{4} \eta-\frac{1}{4} \xi^{4} \eta^{2}-\frac{1}{16} \xi^{4} \eta^{3}+\frac{1}{8} \xi^{4} \eta^{4} \\
& \bar{H}_{3}=\frac{3}{16} \xi \eta-\frac{3}{16} \xi \eta^{2}-\frac{3}{16} \xi \eta^{3}+\frac{3}{16} \xi \eta^{4}-\frac{1}{4} \xi^{2} \eta+\frac{1}{4} \xi^{2} \eta^{2}+\frac{1}{4} \xi^{2} \eta^{3}-\frac{1}{4} \xi^{2} \eta^{4}-\frac{1}{16} \xi^{3} \eta+ \\
& \frac{1}{16} \xi^{3} \eta^{2}+\frac{1}{16} \xi^{3} \eta^{3}-\frac{1}{16} \xi^{3} \eta^{4}+\frac{1}{8} \xi^{4} \eta-\frac{1}{8} \xi^{4} \eta^{2}-\frac{1}{8} \xi^{4} \eta^{3}+\frac{1}{8} \xi^{4} \eta^{4} \\
& \bar{H}_{4}=\frac{1}{16} \xi \eta-\frac{1}{16} \xi \eta^{2}-\frac{1}{16} \xi \eta^{3}+\frac{1}{16} \xi \eta^{4}-\frac{1}{16} \xi^{2} \eta+\frac{1}{16} \xi^{2} \eta^{2}+\frac{1}{16} \xi^{2} \eta^{3}-\frac{1}{16} \xi^{2} \eta^{4}-\frac{1}{16} \xi^{3} \eta+ \\
& \frac{1}{16} \xi^{3} \eta^{2}+\frac{1}{16} \xi^{3} \eta^{3}-\frac{1}{16} \xi^{3} \eta^{4}+\frac{1}{16} \xi^{4} \eta-\frac{1}{16} \xi^{4} \eta^{2}-\frac{1}{16} \xi^{4} \eta^{3}+\frac{1}{16} \xi^{4} \eta^{4} \\
& \bar{H}_{5}=-\frac{9}{16} \xi \eta+\frac{3}{4} \xi \eta^{2}+\frac{3}{16} \xi \eta^{3}-\frac{3}{8} \xi \eta^{4}-\frac{3}{4} \xi^{2} \eta+\xi^{2} \eta^{2}+\frac{1}{4} \xi^{2} \eta^{3}-\frac{1}{2} \xi^{2} \eta^{4}+\frac{3}{16} \xi^{3} \eta-\frac{1}{4} \xi^{3} \eta^{2} \\
& -\frac{1}{16} \xi^{3} \eta^{3}+\frac{1}{8} \xi^{3} \eta^{4}+\frac{3}{8} \xi^{4} \eta-\frac{1}{2} \xi^{4} \eta^{2}-\frac{1}{8} \xi^{4} \eta^{3}+\frac{1}{4} \xi^{4} \eta^{4} \\
& \bar{H}_{6}=\frac{3}{16} \xi \eta-\frac{1}{4} \xi \eta^{2}-\frac{1}{16} \xi \eta^{3}+\frac{1}{8} \xi \eta^{4}+\frac{3}{16} \xi^{2} \eta-\frac{1}{4} \xi^{2} \eta^{2}-\frac{1}{16} \xi^{2} \eta^{3}+\frac{1}{8} \xi^{2} \eta^{4}-\frac{3}{16} \xi^{3} \eta+ \\
& \frac{1}{4} \xi^{3} \eta^{2}+\frac{1}{16} \xi^{3} \eta^{3}-\frac{1}{8} \xi^{3} \eta^{4}-\frac{3}{16} \xi^{4} \eta+\frac{1}{4} \xi^{4} \eta^{2}+\frac{1}{16} \xi^{4} \eta^{3}-\frac{1}{8} \xi^{4} \eta^{4} \\
& \bar{H}_{7}=-\frac{3}{16} \xi \eta+\frac{3}{16} \xi \eta^{2}+\frac{3}{16} \xi \eta^{3}-\frac{3}{16} \xi \eta^{4}-\frac{1}{4} \xi^{2} \eta+\frac{1}{4} \xi^{2} \eta^{2}+\frac{1}{4} \xi^{2} \eta^{3}-\frac{1}{4} \xi^{2} \eta^{4}+\frac{1}{16} \xi^{3} \eta \\
& -\frac{1}{16} \xi^{3} \eta^{2}-\frac{1}{16} \xi^{3} \eta^{3}+\frac{1}{16} \xi^{3} \eta^{4}+\frac{1}{8} \xi^{4} \eta-\frac{1}{8} \xi^{4} \eta^{2}-\frac{1}{8} \xi^{4} \eta^{3}+\frac{1}{8} \xi^{4} \eta^{4} \\
& \bar{H}_{8}=\frac{1}{16} \xi \eta-\frac{1}{16} \xi \eta^{2}-\frac{1}{16} \xi \eta^{3}+\frac{1}{16} \xi \eta^{4}+\frac{1}{16} \xi^{2} \eta-\frac{1}{16} \xi^{2} \eta^{2}-\frac{1}{16} \xi^{2} \eta^{3}+\frac{1}{16} \xi^{2} \eta^{4}-\frac{1}{16} \xi^{3} \eta+ \\
& \frac{1}{16} \xi^{3} \eta^{2}+\frac{1}{16} \xi^{3} \eta^{3}-\frac{1}{16} \xi^{3} \eta^{4}-\frac{1}{16} \xi^{4} \eta+\frac{1}{16} \xi^{4} \eta^{2}+\frac{1}{16} \xi^{4} \eta^{3}-\frac{1}{16} \xi^{4} \eta^{4} \\
& \bar{H}_{9}=\frac{9}{16} \xi \eta+\frac{3}{4} \xi \eta^{2}-\frac{3}{16} \xi \eta^{3}-\frac{3}{8} \xi \eta^{4}+\frac{3}{4} \xi^{2} \eta+\xi^{2} \eta^{2}-\frac{1}{4} \xi^{2} \eta^{3}-\frac{1}{2} \xi^{2} \eta^{4}-\frac{3}{16} \xi^{3} \eta-\frac{1}{4} \xi^{3} \eta^{2}+ \\
& \frac{1}{16} \xi^{3} \eta^{3}+\frac{1}{8} \xi^{3} \eta^{4}-\frac{3}{8} \xi^{4} \eta-\frac{1}{2} \xi^{4} \eta^{2}+\frac{1}{8} \xi^{4} \eta^{3}+\frac{1}{4} \xi^{4} \eta^{4} \\
& \bar{H}_{10}=-\frac{3}{16} \xi \eta-\frac{1}{4} \xi \eta^{2}+\frac{1}{16} \xi \eta^{3}+\frac{1}{8} \xi \eta^{4}-\frac{3}{16} \xi^{2} \eta-\frac{1}{4} \xi^{2} \eta^{2}+\frac{1}{16} \xi^{2} \eta^{3}+\frac{1}{8} \xi^{2} \eta^{4}+\frac{3}{16} \xi^{3} \eta+ \\
& \frac{1}{4} \xi^{3} \eta^{2}-\frac{1}{16} \xi^{3} \eta^{3}-\frac{1}{8} \xi^{3} \eta^{4}+\frac{3}{16} \xi^{4} \eta+\frac{1}{4} \xi^{4} \eta^{2}-\frac{1}{16} \xi^{4} \eta^{3}-\frac{1}{8} \xi^{4} \eta^{4} \\
& \bar{H}_{11}=-\frac{3}{16} \xi \eta-\frac{3}{16} \xi \eta^{2}+\frac{3}{16} \xi \eta^{3}+\frac{3}{16} \xi \eta^{4}-\frac{1}{4} \xi^{2} \eta-\frac{1}{4} \xi^{2} \eta^{2}+\frac{1}{4} \xi^{2} \eta^{3}+\frac{1}{4} \xi^{2} \eta^{4}+\frac{1}{16} \xi^{3} \eta+ \\
& \frac{1}{16} \xi^{3} \eta^{2}-\frac{1}{16} \xi^{3} \eta^{3}-\frac{1}{16} \xi^{3} \eta^{4}+\frac{1}{8} \xi^{4} \eta+\frac{1}{8} \xi^{4} \eta^{2}-\frac{1}{8} \xi^{4} \eta^{3}-\frac{1}{8} \xi^{4} \eta^{4} \\
& \bar{H}_{12}=\frac{1}{16} \xi \eta+\frac{1}{16} \xi \eta^{2}-\frac{1}{16} \xi \eta^{3}-\frac{1}{16} \xi \eta^{4}+\frac{1}{16} \xi^{2} \eta+\frac{1}{16} \xi^{2} \eta^{2}-\frac{1}{16} \xi^{2} \eta^{3}-\frac{1}{16} \xi^{2} \eta^{4}-\frac{1}{16} \xi^{3} \eta-
\end{aligned}
$$




$$
\begin{aligned}
& \frac{1}{16} \xi^{3} \eta^{2}+\frac{1}{16} \xi^{3} \eta^{3}+\frac{1}{16} \xi^{3} \eta^{4}-\frac{1}{16} \xi^{4} \eta-\frac{1}{16} \xi^{4} \eta^{2}+\frac{1}{16} \xi^{4} \eta^{3}+\frac{1}{16} \xi^{4} \eta^{4} \\
& \bar{H}_{13}=-\frac{9}{16} \xi \eta-\frac{3}{4} \xi \eta^{2}+\frac{3}{16} \xi \eta^{3}+\frac{3}{8} \xi \eta^{4}+\frac{3}{4} \xi^{2} \eta+\xi^{2} \eta^{2}-\frac{1}{4} \xi^{2} \eta^{3}-\frac{1}{2} \xi^{2} \eta^{4}+\frac{3}{16} \xi^{3} \eta+\frac{1}{4} \xi^{3} \eta^{2} \\
& -\frac{1}{16} \xi^{3} \eta^{3}-\frac{1}{8} \xi^{3} \eta^{4}-\frac{3}{8} \xi^{4} \eta-\frac{1}{2} \xi^{4} \eta^{2}+\frac{1}{8} \xi^{4} \eta^{3}+\frac{1}{4} \xi^{4} \eta^{4} \\
& \bar{H}_{14}=-\frac{3}{16} \xi \eta-\frac{1}{4} \xi \eta^{2}+\frac{1}{16} \xi \eta^{3}+\frac{1}{8} \xi \eta^{4}+\frac{3}{16} \xi^{2} \eta+\frac{1}{4} \xi^{2} \eta^{2}-\frac{1}{16} \xi^{2} \eta^{3}-\frac{1}{8} \xi^{2} \eta^{4}+\frac{3}{16} \xi^{3} \eta+ \\
& \frac{1}{4} \xi^{3} \eta^{2}-\frac{1}{16} \xi^{3} \eta^{3}-\frac{1}{8} \xi^{3} \eta^{4}-\frac{3}{16} \xi^{4} \eta-\frac{1}{4} \xi^{4} \eta^{2}+\frac{1}{16} \xi^{4} \eta^{3}+\frac{1}{8} \xi^{4} \eta^{4} \\
& \bar{H}_{15}=\frac{3}{16} \xi \eta+\frac{3}{16} \xi \eta^{2}-\frac{3}{16} \xi \eta^{3}-\frac{3}{16} \xi \eta^{4}-\frac{1}{4} \xi^{2} \eta-\frac{1}{4} \xi^{2} \eta^{2}+\frac{1}{4} \xi^{2} \eta^{3}+\frac{1}{4} \xi^{2} \eta^{4}-\frac{1}{16} \xi^{3} \eta \\
& -\frac{1}{16} \xi^{3} \eta^{2}+\frac{1}{16} \xi^{3} \eta^{3}+\frac{1}{16} \xi^{3} \eta^{4}+\frac{1}{8} \xi^{4} \eta+\frac{1}{8} \xi^{4} \eta^{2}-\frac{1}{8} \xi^{4} \eta^{3}-\frac{1}{8} \xi^{4} \eta^{4} \\
& \bar{H}_{16}=\frac{1}{16} \xi \eta+\frac{1}{16} \xi \eta^{2}-\frac{1}{16} \xi \eta^{3}-\frac{1}{16} \xi \eta^{4}-\frac{1}{16} \xi^{2} \eta-\frac{1}{16} \xi^{2} \eta^{2}+\frac{1}{16} \xi^{2} \eta^{3}+\frac{1}{16} \xi^{2} \eta^{4}-\frac{1}{16} \xi^{3} \eta- \\
& \frac{1}{16} \xi^{3} \eta^{2}+\frac{1}{16} \xi^{3} \eta^{3}+\frac{1}{16} \xi^{3} \eta^{4}+\frac{1}{16} \xi^{4} \eta+\frac{1}{16} \xi^{4} \eta^{2}-\frac{1}{16} \xi^{4} \eta^{3}-\frac{1}{16} \xi^{4} \eta^{4} \\
& \bar{H}_{17}=-\frac{3}{4} \eta+\eta^{2}+\frac{1}{4} \eta^{3}-\frac{1}{2} \eta^{4}+\frac{3}{2} \xi^{2} \eta-2 \xi^{2} \eta^{2}-\frac{1}{2} \xi^{2} \eta^{3}+\xi^{2} \eta^{4}-\frac{3}{4} \xi^{4} \eta+\xi^{4} \eta^{2}+\frac{1}{4} \xi^{4} \eta^{3}- \\
& \frac{1}{2} \xi^{4} \eta^{4} \\
& \bar{H}_{18}=-\frac{1}{4} \eta+\frac{1}{4} \eta^{2}+\frac{1}{4} \eta^{3}-\frac{1}{4} \eta^{4}+\frac{1}{2} \xi^{2} \eta-\frac{1}{2} \xi^{2} \eta^{2}-\frac{1}{2} \xi^{2} \eta^{3}+\frac{1}{2} \xi^{2} \eta^{4}-\frac{1}{4} \xi^{4} \eta+\frac{1}{4} \xi^{4} \eta^{2}+ \\
& \frac{1}{4} \xi^{4} \eta^{3}-\frac{1}{4} \xi^{4} \eta^{4} \\
& \bar{H}_{19}=\frac{3}{4} \xi-\frac{3}{2} \xi \eta^{2}+\frac{3}{4} \xi \eta^{4}+\xi^{2}-2 \xi^{2} \eta^{2}+\xi^{2} \eta^{4}-\frac{1}{4} \xi^{3}+\frac{1}{2} \xi^{3} \eta^{2}-\frac{1}{4} \xi^{3} \eta^{4}-\frac{1}{2} \xi^{4}+\xi^{4} \eta^{2}- \\
& \frac{1}{2} \xi^{4} \eta^{4} \\
& \bar{H}_{20}=-\frac{1}{4} \xi+\frac{1}{2} \xi \eta^{2}-\frac{1}{4} \xi \eta^{4}-\frac{1}{4} \xi^{2}+\frac{1}{2} \xi^{2} \eta^{2}-\frac{1}{4} \xi^{2} \eta^{4}+\frac{1}{4} \xi^{3}-\frac{1}{2} \xi^{3} \eta^{2}+\frac{1}{4} \xi^{3} \eta^{4}+\frac{1}{4} \xi^{4}- \\
& \frac{1}{2} \xi^{4} \eta^{2}+\frac{1}{4} \xi^{4} \eta^{4} \\
& \bar{H}_{21}=\frac{3}{4} \eta+\eta^{2}-\frac{1}{4} \eta^{3}-\frac{1}{2} \eta^{4}-\frac{3}{2} \xi^{2} \eta-2 \xi^{2} \eta^{2}+\frac{1}{2} \xi^{2} \eta^{3}+\xi^{2} \eta^{4}+\frac{3}{4} \xi^{4} \eta+\xi^{4} \eta^{2}-\frac{1}{4} \xi^{4} \eta^{3}- \\
& \frac{1}{2} \xi^{4} \eta^{4} \\
& \bar{H}_{22}=-\frac{1}{4} \eta-\frac{1}{4} \eta^{2}+\frac{1}{4} \eta^{3}+\frac{1}{4} \eta^{4}+\frac{1}{2} \xi^{2} \eta+\frac{1}{2} \xi^{2} \eta^{2}-\frac{1}{2} \xi^{2} \eta^{3}-\frac{1}{2} \xi^{2} \eta^{4}-\frac{1}{4} \xi^{4} \eta-\frac{1}{4} \xi^{4} \eta^{2}+ \\
& \frac{1}{4} \xi^{4} \eta^{3}+\frac{1}{4} \xi^{4} \eta^{4} \\
& \bar{H}_{23}=-\frac{3}{4} \xi+\frac{3}{2} \xi \eta^{2}-\frac{3}{4} \xi \eta^{4}+\xi^{2}-2 \xi^{2} \eta^{2}+\xi^{2} \eta^{4}+\frac{1}{4} \xi^{3}-\frac{1}{2} \xi^{3} \eta^{2}+\frac{1}{4} \xi^{3} \eta^{4}-\frac{1}{2} \xi^{4}+\xi^{4} \eta^{2}- \\
& \frac{1}{2} \xi^{4} \eta^{4} \\
& \bar{H}_{24}=-\frac{1}{4} \xi+\frac{1}{2} \xi \eta^{2}-\frac{1}{4} \xi \eta^{4}+\frac{1}{4} \xi^{2}-\frac{1}{2} \xi^{2} \eta^{2}+\frac{1}{4} \xi^{2} \eta^{4}+\frac{1}{4} \xi^{3}-\frac{1}{2} \xi^{3} \eta^{2}+\frac{1}{4} \xi^{3} \eta^{4}-\frac{1}{4} \xi^{4}+ \\
& \frac{1}{2} \xi^{4} \eta^{2}-\frac{1}{4} \xi^{4} \eta^{4}
\end{aligned}
$$


$\bar{H}_{25}=1-2 \eta^{2}+\eta^{4}-2 \xi^{2}+4 \xi^{2} \eta^{2}-2 \xi^{2} \eta^{4}+\xi^{4}-2 \xi^{4} \eta^{2}+\xi^{4} \eta^{4}$

The set of Hermite shape functions is

$$
\lfloor\bar{H}(\xi, \eta)\rfloor=\left\lfloor\begin{array}{llll}
\bar{H}_{1} & \bar{H}_{2} & \ldots & \bar{H}_{25}
\end{array}\right\rfloor
$$

The bending lateral displacements are given by

$$
\begin{aligned}
w_{b}(\xi, \eta) & =\lfloor\bar{H}\rfloor\left\{\bar{q}_{w_{b}}\right\} \\
\frac{\partial w_{b}}{\partial \xi}(\xi, \eta) & =\left\lfloor\frac{\partial \bar{H}}{\partial \xi}\right\rfloor\left\{\bar{q}_{w_{b}}\right\} \\
\frac{\partial w_{b}}{\partial \eta}(\xi, \eta) & =\left\lfloor\frac{\partial \bar{H}}{\partial \eta}\right\rfloor\left\{\bar{q}_{w_{b}}\right\} \\
\frac{\partial w_{b}^{2}}{\partial \xi \partial \eta}(\xi, \eta) & =\left\lfloor\frac{\partial \bar{H}}{\partial \xi \partial \eta}\right\rfloor\left\{\bar{q}_{w_{b}}\right\}
\end{aligned}
$$

where $\left\{\bar{q}_{w_{b}}\right\}$ is a bending lateral displacement degree of freedom vector and the bar accent represents the standard domain. The shear lateral displacements are similarly calculated. The finite element displacements in the standard domain must now be transformed for use in the physical domain.

\subsubsection{MONNA Plate Transformation}

It has been established that rectangular $C^{1}$ continuous plate elements have difficulty with quadrilateral transformation [20]. Despite many attempts to derive a higher-order element with this ability, the present element also does not directly have the capacity for quadrilateral shapes in the physical domain. The reason for this deficiency is demonstrated in the attempt to perform the transformation.

There are two transformations that must occur: the transformation of the degree of freedom vector and the transformation of the shape function differentiation. The problem lies with the first transformation, which involves associating the Hermite shape functions with physical DOF instead of standard DOF. Consider the expanded expression of a bending lateral displacement in the standard domain from Eq. (2.69),

$$
w_{b}(\xi, \eta)=\bar{H}_{1} w_{1}+\bar{H}_{2} \frac{\partial w_{1}}{\partial \xi}+\bar{H}_{3} \frac{\partial w_{1}}{\partial \xi}+\bar{H}_{4} \frac{\partial^{2} w_{1}}{\partial \xi \partial \eta}+\ldots+\bar{H}_{25} w_{9}
$$


Transforming the standard domain DOF using Eqs. (2.47) and (2.56), the expression becomes

$$
\begin{aligned}
w_{b}(\xi, \eta)= & \bar{H}_{1} w_{1}+\bar{H}_{2}\left(J_{11} \frac{\partial w_{1}}{\partial x}+J_{12} \frac{\partial w_{1}}{\partial y}\right)+\bar{H}_{3}\left(J_{21} \frac{\partial w_{1}}{\partial x}+J_{22} \frac{\partial w_{1}}{\partial y}\right)+ \\
& \bar{H}_{4}\left(J_{11}^{\eta} \frac{\partial w_{1}}{\partial x}+J_{12}^{\eta} \frac{\partial w_{1}}{\partial y}+J_{11} J_{21} \frac{\partial^{2} w_{1}}{\partial x^{2}}+J_{12} J_{22} \frac{\partial^{2} w_{1}}{\partial y^{2}}+\left(J_{12} J_{21}+J_{11} J_{22}\right) \frac{\partial^{2} w_{1}}{\partial x \partial y}\right)+ \\
& \ldots+\bar{H}_{25} w_{9}
\end{aligned}
$$

Next, the expression is rearranged to collect like-terms,

$$
\begin{aligned}
w_{b}(\xi, \eta)= & \bar{H}_{1} w_{1}+\left(\bar{H}_{2} J_{11}+\bar{H}_{3} J_{21}+\bar{H}_{4} J_{11}^{\eta}\right) \frac{\partial w_{1}}{\partial x}+\left(\bar{H}_{2} J_{12}+\bar{H}_{3} J_{22}+\bar{H}_{4} J_{12}^{\eta}\right) \frac{\partial w_{1}}{\partial y}+ \\
& \bar{H}_{4} J_{11} J_{21} \frac{\partial^{2} w_{1}}{\partial x^{2}}+\bar{H}_{4} J_{12} J_{22} \frac{\partial^{2} w_{1}}{\partial y^{2}}+\bar{H}_{4}\left(J_{12} J_{21}+J_{11} J_{22}\right) \frac{\partial^{2} w_{1}}{\partial x \partial y}+\ldots+\bar{H}_{25} w_{9}
\end{aligned}
$$

There are a couple problems that are evident. First, the slopes in the standard domain are a function of both physical domain slopes. This is a problem because midside nodes in the MONNA plate element include only one slope. Secondly, the cross derivative transformation introduces second derivative DOF that are not present in the formulation.

Therefore, it may be concluded when any slope DOF is present at a given node, quadrilateral transformation for a rectangular $C^{1}$ continuous element requires that both slope DOF $\left(\frac{\partial w_{b}}{\partial \xi}, \frac{\partial w_{b}}{\partial \eta}\right)$ be present at that node. Similarly, both second derivative DOF $\left(\frac{\partial^{2} w_{b}}{\partial \xi^{2}}, \frac{\partial^{2} w_{b}}{\partial \eta^{2}}\right)$ must also be included where cross derivatives are present. Many attempts have been made to derive Hermite shape functions for MONNA while including both slopes at each midside node and including second derivatives wherever cross derivatives are present, however, including these DOF caused singularities in the derivation process. The only higher-order DOF distribution found to allow a successful Hermite shape function derivation, using the method described, is that presented in Eq. (2.69). This distribution does not include both slopes at the midside nodes or second derivatives such that quadrilateral transformation is not possible.

Nevertheless, the MONNA element is a conforming element. It is conforming because the normal slope varies quartically along a boundary and there are at least four degrees of freedom available to define each boundary's normal slope variation. Thus, is it concluded that the MONNA plate element is conforming, however, it does not directly satisfy the requirements for quadrilateral transformation. 


\section{Rectangular Transformation}

The MONNA element is then currently only applicable to rectangular element shapes, similar to other $C^{1}$ rectangular plate elements. For rectangular element shapes, $J_{12}, J_{21}$, $J_{11}^{\eta}$, and $J_{12}^{\eta}$ terms are zero, which greatly simplifies the transformation. Reconsider Eq. (2.72) for rectangular shapes,

$$
w_{b}(\xi, \eta)=\bar{H}_{1} w_{1}+\bar{H}_{2} J_{11} \frac{\partial w_{1}}{\partial x}+\bar{H}_{3} J_{22} \frac{\partial w_{1}}{\partial y}+\bar{H}_{4} J_{11} J_{22} \frac{\partial w_{1}^{2}}{\partial x \partial y}+\ldots+\bar{H}_{25} w_{9}
$$

Transformed shape functions associated with physical DOF are introduced as

$$
\begin{aligned}
H_{1} & =\bar{H}_{1} \\
H_{2} & =J_{11} \bar{H}_{2} \\
H_{3} & =J_{22} \bar{H}_{3} \\
H_{4} & =J_{11} J_{22} \bar{H}_{4} \\
\vdots & \\
H_{25} & =\bar{H}_{25}
\end{aligned}
$$

This leads to an updated set of Hermite shape functions associated with physical degrees of freedom, $\left\{q_{w}\right\}$,

$$
\lfloor H(\xi, \eta)\rfloor=\left\lfloor\begin{array}{llll}
H_{1} & H_{2} & \ldots & H_{25}
\end{array}\right.
$$

Thus, the equivalence between displacement in terms of standard and physical DOF is

$$
w_{b}=\lfloor\bar{H}(\xi, \eta)\rfloor\left\{\bar{q}_{w_{b}}\right\}=\lfloor H(\xi, \eta)\rfloor\left\{q_{w_{b}}\right\}
$$

Next, the differentiation of the shape functions must be transformed. The inverse transformations defined in Eqs. (2.58) and (2.59) are used for this purpose, where $J_{12}^{*}, J_{21}^{*}$, and the $T_{A}$ matrix are zero for rectangular shapes. The in-plane displacements require only first derivatives, so if $H_{L}(x, y)$ is a given Lagrange shape function, the first derivative transformations are given by

$$
\begin{aligned}
\frac{\partial H_{L}}{\partial x} & =J_{11}^{*} \frac{\partial H_{L}}{\partial \xi} \\
\frac{\partial H_{L}}{\partial y} & =J_{22}^{*} \frac{\partial H_{L}}{\partial \eta}
\end{aligned}
$$


The lateral displacements require both first and second derivatives. If $H(x, y)$ is a given Hermite shape function, then the transformations are given by

$$
\begin{aligned}
\frac{\partial H}{\partial x} & =J_{11}^{*} \frac{\partial H}{\partial \xi} \\
\frac{\partial H}{\partial y} & =J_{22}^{*} \frac{\partial H}{\partial \eta} \\
\frac{\partial^{2} H}{\partial x^{2}} & =\left(J_{11}^{*}\right)^{2} \frac{\partial^{2} H}{\partial \xi^{2}} \\
\frac{\partial^{2} H}{\partial y^{2}} & =\left(J_{22}^{*}\right)^{2} \frac{\partial^{2} H}{\partial \eta^{2}} \\
\frac{\partial^{2} H}{\partial x \partial y} & =J_{11}^{*} J_{22}^{*} \frac{\partial^{2} H}{\partial \xi \partial \eta}
\end{aligned}
$$

\subsubsection{Element Stiffness Matrix Formulation}

The finite element stiffness is derived from the virtual strain energy, $\delta U$. The relationship between the virtual strain energy and the element stiffness matrix is

$$
\delta U_{e}=\left\lfloor\delta q_{e}\right\rfloor\left[k_{e}\right]\left\{q_{e}\right\}
$$

where $\left\{q_{e}\right\}$ is the element degree of freedom vector and $\left[k_{e}\right]$ is the element stiffness matrix. The real and virtual displacements in the virtual strain energy equation are expressed in terms of shape function and degree of freedom vectors. For example, the real and virtual expressions for in-plane displacement, $u$, are respectively,

$$
\begin{aligned}
u & =\left\lfloor H_{L}\right\rfloor\left\{q_{u}\right\} \\
\delta u & =\left\lfloor\delta q_{u}\right\rfloor\left\{H_{L}\right\}
\end{aligned}
$$

The real and virtual expressions for the bending lateral displacement, $w_{b}$, are respectively,

$$
\begin{aligned}
w_{b} & =\lfloor H\rfloor\left\{q_{w_{b}}\right\} \\
\delta w_{b} & =\left\lfloor\delta q_{w_{b}}\right\rfloor\{H\}
\end{aligned}
$$

Similarly, the real and virtual expressions for the other variables are written. The MONNA plate element has 68 degrees of freedom, so the element stiffness matrix is of size $(68 \times 68)$. There are four independent variables: $u, v, w_{b}$, and $w_{s}$. The stiffness 
matrix is symmetric about the diagonal and organized into submatrices as

$$
\left[k_{e}\right]=\left[\begin{array}{cccc}
{\left[k_{u u}\right]} & {\left[k_{u v}\right]} & {\left[k_{u w_{b}}\right]} & {\left[k_{u w_{s}}\right]} \\
{\left[k_{u v}\right]^{T}} & {\left[k_{v v}\right]} & {\left[k_{v w_{b}}\right]} & {\left[k_{v w_{s}}\right]} \\
{\left[k_{u w_{b}}\right]^{T}} & {\left[k_{v w_{b}}\right]^{T}} & {\left[k_{w_{b} w_{b}}\right]} & {\left[k_{w_{b} w_{s}}\right]} \\
{\left[k_{u w_{s}}\right]^{T}} & {\left[k_{v w_{s}}\right]^{T}} & {\left[k_{w_{b} w_{s}}\right]^{T}} & {\left[k_{w_{s} w_{s}}\right]}
\end{array}\right]
$$

The stiffness submatrices are derived from the virtual strain energy in Eq. (2.35) using the constitutive relations from Eq. (2.25). The various stiffness submatrices are listed as follows.

$$
\begin{aligned}
& {\left[k_{u u}\right]=\iint_{A}\left(A_{11}\left\{H_{L}^{x}\right\}\left\lfloor H_{L}^{x}\right\rfloor+A_{16}\left\{H_{L}^{x}\right\}\left\lfloor H_{L}^{y}\right\rfloor+A_{16}\left\{H_{L}^{y}\right\}\left\lfloor H_{L}^{x}\right\rfloor+A_{66}\left\{H_{L}^{y}\right\}\left\lfloor H_{L}^{y}\right\rfloor\right) d x d y} \\
& {\left[k_{u v}\right]=\iint_{A}\left(A_{12}\left\{H_{L}^{x}\right\}\left\lfloor H_{L}^{y}\right\rfloor+A_{16}\left\{H_{L}^{x}\right\}\left\lfloor H_{L}^{x}\right\rfloor+A_{26}\left\{H_{L}^{y}\right\}\left\lfloor H_{L}^{y}\right\rfloor+A_{66}\left\{H_{L}^{y}\right\}\left\lfloor H_{L}^{x}\right\rfloor\right) d x d y} \\
& {\left[k_{u w_{b}}\right]=\iint_{A}\left(-B_{11}\left\{H_{L}^{x}\right\}\left\lfloor H^{x x}\right\rfloor-B_{12}\left\{H_{L}^{x}\right\}\left\lfloor H^{y y}\right\rfloor-2 B_{16}\left\{H_{L}^{x}\right\}\left\lfloor H^{x y}\right\rfloor-B_{16}\left\{H_{L}^{y}\right\}\left\lfloor H^{x x}\right\rfloor\right.} \\
& \left.-B_{26}\left\{H_{L}^{y}\right\}\left\lfloor H^{y y}\right\rfloor-2 B_{66}\left\{H_{L}^{y}\right\}\left\lfloor H^{x y}\right\rfloor\right) d x d y \\
& {\left[k_{u w_{s}}\right]=\iint_{A} c_{1}\left(-E_{11}\left\{H_{L}^{x}\right\}\left\lfloor H^{x x}\right\rfloor-E_{12}\left\{H_{L}^{x}\right\}\left\lfloor H^{y y}\right\rfloor-2 E_{16}\left\{H_{L}^{x}\right\}\left\lfloor H^{x y}\right\rfloor-E_{16}\left\{H_{L}^{y}\right\}\left\lfloor H^{x x}\right\rfloor\right.} \\
& \left.-E_{26}\left\{H_{L}^{y}\right\}\left\lfloor H^{y y}\right\rfloor-2 E_{66}\left\{H_{L}^{y}\right\}\left\lfloor H^{x y}\right\rfloor\right) d x d y \\
& {\left[k_{v v}\right]=\iint_{A}\left(A_{22}\left\{H_{L}^{y}\right\}\left\lfloor H_{L}^{y}\right\rfloor+A_{26}\left\{H_{L}^{y}\right\}\left\lfloor H_{L}^{x}\right\rfloor+A_{26}\left\{H_{L}^{x}\right\}\left\lfloor H_{L}^{y}\right\rfloor+A_{66}\left\{H_{L}^{x}\right\}\left\lfloor H_{L}^{x}\right\rfloor\right) d x d y} \\
& {\left[k_{v w_{b}}\right]=\iint_{A}\left(-B_{12}\left\{H_{L}^{y}\right\}\left\lfloor H^{x x}\right\rfloor-B_{22}\left\{H_{L}^{y}\right\}\left\lfloor H^{y y}\right\rfloor-2 B_{16}\left\{H_{L}^{y}\right\}\left\lfloor H^{x y}\right\rfloor-B_{16}\left\{H_{L}^{x}\right\}\left\lfloor H^{x x}\right\rfloor\right.} \\
& \left.-B_{26}\left\{H_{L}^{x}\right\}\left\lfloor H^{y y}\right\rfloor-2 B_{66}\left\{H_{L}^{x}\right\}\left\lfloor H^{x y}\right\rfloor\right) d x d y \\
& {\left[k_{v w_{s}}\right]=\iint_{A} c_{1}\left(-E_{12}\left\{H_{L}^{y}\right\}\left\lfloor H^{x x}\right\rfloor-E_{22}\left\{H_{L}^{y}\right\}\left\lfloor H^{y y}\right\rfloor-2 E_{26}\left\{H_{L}^{y}\right\}\left\lfloor H^{x y}\right\rfloor-E_{16}\left\{H_{L}^{x}\right\}\left\lfloor H^{x x}\right\rfloor\right.} \\
& \left.-E_{26}\left\{H_{L}^{x}\right\}\left\lfloor H^{y y}\right\rfloor-2 E_{66}\left\{H_{L}^{x}\right\}\left\lfloor H^{x y}\right\rfloor\right) d x d y \\
& {\left[k_{w_{b} w_{b}}\right]=\iint_{A}\left(N_{x}\left\{H^{x}\right\}\left\lfloor H^{x}\right\rfloor+D_{11}\left\{H^{x x}\right\}\left\lfloor H^{x x}\right\rfloor+D_{12}\left\{H^{x x}\right\}\left\lfloor H^{y y}\right\rfloor+2 D_{16}\left\{H^{x x}\right\}\left\lfloor H^{x y}\right\rfloor\right.} \\
& +N_{y}\left\{H^{y}\right\}\left\lfloor H^{y}\right\rfloor+D_{12}\left\{H^{y y}\right\}\left\lfloor H^{x x}\right\rfloor+D_{22}\left\{H^{y y}\right\}\left\lfloor H^{y y}\right\rfloor+2 D_{26}\left\{H^{y y}\right\}\left\lfloor H^{x y}\right\rfloor \\
& \left.+2 D_{16}\left\{H^{x y}\right\}\left\lfloor H^{x x}\right\rfloor+2 D_{26}\left\{H^{x y}\right\}\left\lfloor H^{y y}\right\rfloor+4 D_{66}\left\{H^{x y}\right\}\left\lfloor H^{x y}\right\rfloor\right) d x d y \\
& {\left[k_{w_{b} w_{s}}\right]=\iint_{A} c_{1}\left(F_{11}\left\{H^{x x}\right\}\left\lfloor H^{x x}\right\rfloor+F_{12}\left\{H^{x x}\right\}\left\lfloor H^{y y}\right\rfloor+2 F_{16}\left\{H^{x x}\right\}\left\lfloor H^{x y}\right\rfloor+F_{12}\left\{H^{y y}\right\}\left\lfloor H^{x x}\right\rfloor\right.}
\end{aligned}
$$




$$
\begin{aligned}
& +F_{22}\left\{H^{y y}\right\}\left\lfloor H^{y y}\right\rfloor+2 F_{26}\left\{H^{y y}\right\}\left\lfloor H^{x y}\right\rfloor+2 F_{16}\left\{H^{x y}\right\}\left\lfloor H^{x x}\right\rfloor \\
& \left.+2 F_{26}\left\{H^{x y}\right\}\left\lfloor H^{y y}\right\rfloor+4 F_{66}\left\{H^{x y}\right\}\left\lfloor H^{x y}\right\rfloor\right) d x d y \\
{\left[k_{w_{s} w_{s}}\right]=\iint_{A}[} & N_{x}\left\{H^{x}\right\}\left\lfloor H^{x}\right\rfloor+N_{y}\left\{H^{y}\right\}\left\lfloor H^{y}\right\rfloor+c_{1}^{2}\left(H_{11}\left\{H^{x x}\right\}\left\lfloor H^{x x}\right\rfloor+H_{12}\left\{H^{x x}\right\}\left\lfloor H^{y y}\right\rfloor\right. \\
& +2 H_{16}\left\{H^{x x}\right\}\left\lfloor H^{x y}\right\rfloor+H_{12}\left\{H^{y y}\right\}\left\lfloor H^{x x}\right\rfloor+H_{22}\left\{H^{y y}\right\}\left\lfloor H^{y y}\right\rfloor+2 H_{26}\left\{H^{y y}\right\}\left\lfloor H^{x y}\right\rfloor \\
& \left.+2 H_{16}\left\{H^{x y}\right\}\left\lfloor H^{x x}\right\rfloor+2 H_{26}\left\{H^{x y}\right\}\left\lfloor H^{y y}\right\rfloor+4 H_{66}\left\{H^{x y}\right\}\left\lfloor H^{x y}\right\rfloor\right) \\
& \left.+D_{45}^{*}\left\{H^{x}\right\}\left\lfloor H^{y}\right\rfloor+D_{55}^{*}\left\{H^{x}\right\}\left\lfloor H^{x}\right\rfloor+D_{44}^{*}\left\{H^{y}\right\}\left\lfloor H^{y}\right\rfloor+D_{45}^{*}\left\{H^{y}\right\}\left\lfloor H^{x}\right\rfloor\right] d x d y
\end{aligned}
$$

\subsubsection{Element Inertia Matrix Formulation}

The finite element inertia matrix is derived from the virtual kinetic energy, $\delta T$. The relationship between the virtual kinetic energy and the inertia matrix is

$$
-\delta T_{e}=\left\lfloor\delta q_{e}\right\rfloor\left[M_{e}\right]\left\{q_{e}\right\}
$$

where $\left[M_{e}\right]$ is the element inertia matrix. The real and virtual displacements are written for each variable in the same manner as in Section 2.3.6. The inertia is also of size $(68 \mathrm{x}$ 68 ), symmetric about its diagonal, and organized into submatrices as

$$
\left[M_{e}\right]=\left[\begin{array}{cccc}
{\left[M_{u u}\right]} & {\left[M_{u v}\right]} & {\left[M_{u w_{b}}\right]} & {\left[M_{u w_{s}}\right]} \\
{\left[M_{u v}\right]^{T}} & {\left[M_{v v}\right]} & {\left[M_{v w_{b}}\right]} & {\left[M_{v w_{s}}\right]} \\
{\left[M_{u w_{b}}\right]^{T}} & {\left[M_{v w_{b}}\right]^{T}} & {\left[M_{w_{b} w_{b}}\right]} & {\left[M_{w_{b} w_{s}}\right]} \\
{\left[M_{u w_{s}}\right]^{T}} & {\left[M_{v w_{s}}\right]^{T}} & {\left[M_{w_{b} w_{s}}\right]^{T}} & {\left[M_{w_{s} w_{s}}\right]}
\end{array}\right]
$$

The inertia submatrices are derived from the virtual kinetic energy listed in Eq. (2.43). The various inertia submatrices are listed as follows:

$$
\begin{aligned}
{\left[M_{u u}\right] } & =\iint_{A} I_{0}\left\{H_{L}\right\}\left\lfloor H_{L}\right\rfloor d x d y \\
{\left[M_{u v}\right] } & =[0] \\
{\left[M_{u w_{b}}\right] } & =\iint_{A}-I_{1}\left\{H_{L}\right\}\left\lfloor H^{x}\right\rfloor d x d y \\
{\left[M_{u w_{s}}\right] } & =\iint_{A}-c_{1} I_{3}\left\{H_{L}\right\}\left\lfloor H^{x}\right\rfloor d x d y
\end{aligned}
$$




$$
\begin{aligned}
{\left[M_{v v}\right] } & =\iint_{A} I_{0}\left\{H_{L}\right\}\left\lfloor H_{L}\right\rfloor d x d y \\
{\left[M_{v w_{b}}\right] } & =\iint_{A}-I_{1}\left\{H_{L}\right\}\left\lfloor H^{y}\right\rfloor d x d y \\
{\left[M_{v w_{s}}\right] } & =\iint_{A}-c_{1} I_{3}\left\{H_{L}\right\}\left\lfloor H^{y}\right\rfloor d x d y \\
{\left[M_{w_{b} w_{b}}\right] } & =\iint_{A}\left(I_{2}\left\{H^{x}\right\}\left\lfloor H^{x}\right\rfloor+I_{2}\left\{H^{y}\right\}\left\lfloor H^{y}\right\rfloor+I_{0}\{H\}\lfloor H\rfloor\right) d x d y \\
{\left[M_{w_{b} w_{s}}\right] } & =\iint_{A} c_{1}\left(I_{4}\left\{H^{x}\right\}\left\lfloor H^{x}\right\rfloor+I_{4}\left\{H^{y}\right\}\left\lfloor H^{y}\right\rfloor+I_{0}\{H\}\lfloor H\rfloor\right) d x d y \\
{\left[M_{w_{s} w_{s}}\right] } & =\iint_{A} c_{1}^{2}\left(I_{6}\left\{H^{x}\right\}\left\lfloor H^{x}\right\rfloor+I_{6}\left\{H^{y}\right\}\left\lfloor H^{y}\right\rfloor+I_{0}\{H\}\lfloor H\rfloor\right) d x d y
\end{aligned}
$$

\subsubsection{Traditional Plate Elements}

The effectiveness of the MONNA plate element in an aeroelasticity context is determined by comparison with the performance of traditional elements. There are two well-established rectangular $C^{1}$ plate finite elements: the non-conforming Melosh element [21] and the conforming Boxer, Fox and Schmidt (BFS) element [22].

\section{Melosh Plate Element}

The Melosh element is a four-node rectangular element with three types of lateral displacement variables at each node: $w, \frac{\partial w}{\partial x}$, and $\frac{\partial w}{\partial y}$. The DOF distribution for the present HSDT composite Melosh plate element is shown in Figure 2.6. This element has

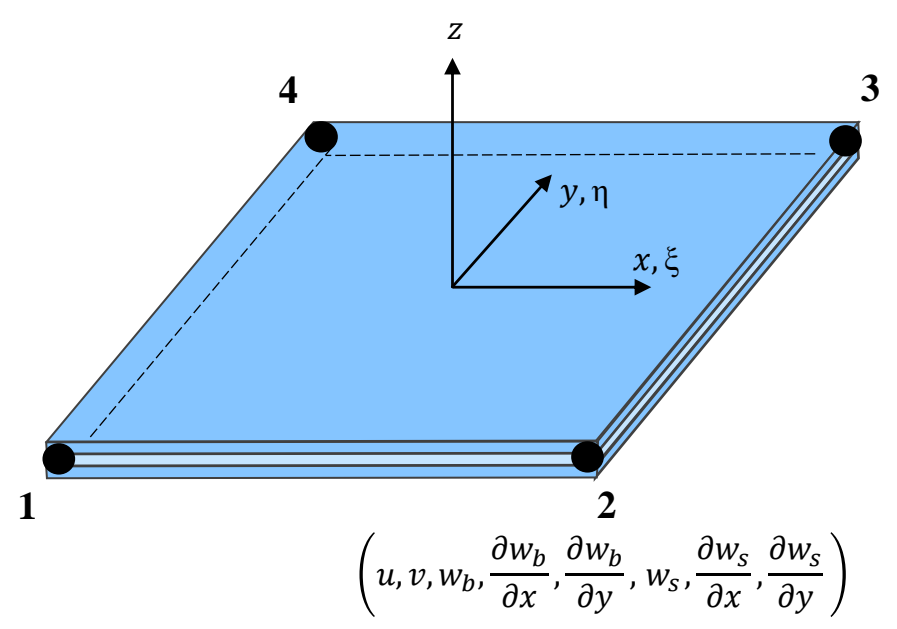

Figure 2.6: A Melosh HSDT plate element with DOF distribution 
a total of 32 degrees of freedom and is an $h$-version element because accuracy is only increased by increasing the number of elements. Unfortunately, this element is non-conforming because the normal slope, $\partial w / \partial n$, varies cubically along a boundary and there are only two normal slope values available on each boundary. Non-conforming elements may have difficulty with convergence and don't entail genuine $C^{1}$ continuity.

Although the Melosh element suffers from non-conformity, its advantage is its capacity for quadrilateral transformation. This transformation is possible because both slopes are included at every node and cross derivatives are not included. Using the same procedure outlined in the MONNA plate transformation of section 2.3.5, the quadrilateral transformation may be successfully performed.

\section{BFS Plate Element}

The BFS element is a four-node rectangular element with four types of lateral displacement variables at each node $w, \frac{\partial w}{\partial x}, \frac{\partial w}{\partial y}$, and $\frac{\partial^{2} w}{\partial x \partial y}$. The DOF distribution for the HSDT composite BFS plate element used in the present analysis is shown in Figure 2.7. This element has a total of 40 degrees of freedom and is also an $h$-version element. As

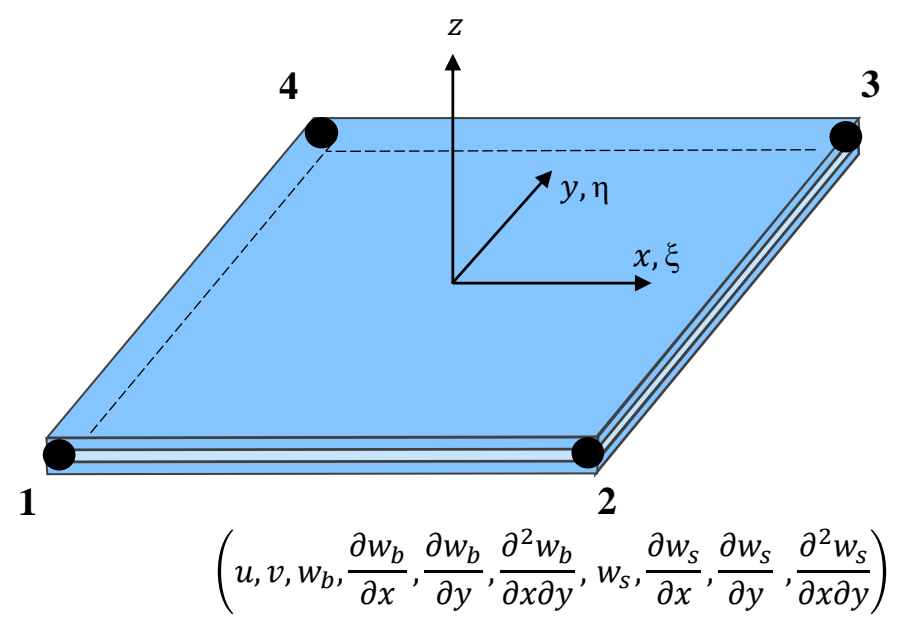

Figure 2.7: A BFS HSDT plate element with DOF distribution

opposed to the Melosh element, the BFS element is conforming because the normal slope varies cubically and there at least three available DOF along each boundary to define the cubic variation. However, this element is limited to rectangular shapes just like the MONNA plate element because the cross derivative transformation introduces second derivatives. The addition of the cross derivatives allows genuine $C^{1}$ continuity and avoids the convergence issues of the Melosh element, but this addition simultaneously eliminates the capacity for quadrilateral transformation. 


\section{Chapter 3}

\section{Aerodynamic Models}

\subsection{Introduction}

The aerodynamic model is a critical component of any aeroelastic analysis. There are multiple approaches available to model steady or unsteady aeroelastic aerodynamics. These include [2]:

1. Strip theory

2. Panel methods

3. Computational fluid dynamics (CFD)

The strip theory is the simplest way of modeling lift distribution; however, this approach does not account for the interacting effects between different parts of the lifting surface. This method is primarily used for crude aerodynamic loading estimations in a design process. Computational fluid dynamics has been increasingly employed in research and industry for more detailed aeroelastic analysis; however, in flutter analysis, CFD models are often applied in industry only as a correction to account for transonic compressibilty effects [2]. The application of panel methods remain the most common and practical approach to modeling divergence and flutter aerodynamics.

Panel methods model the lift distribution by discretizing a lifting surface into a series of panels with potential flow aerodynamic elements that are related through Influence Coefficients (IC) [2]. These methods are used to model the aerodynamics in the present aeroelastic analysis because they offer an efficient balance of accuracy and simplicity. The current chapter explores each of the steady and unsteady panel methods used in this thesis. 


\subsection{Steady Aerodynamics}

Steady aeroelastic analysis, such as divergence, requires steady aerodynamic loading. Although unsteady aerodynamic models may be used to calculate steady aerodynamic loads, stand-alone steady panel methods remain valuable because they can improve the steady component of unsteady models.

\subsubsection{Vortex Lattice Method}

The classical Vortex Lattice Method (VLM) is one of the most accurate and widely used steady panel methods. It is valid for incompressible, inviscid, and irrotational flow. The half-span is discretized into a series of spanwise and chordwise panels of number $n$, each with a horseshoe vortex fixed at the 1/4-chord location as shown in Figure 3.1. The

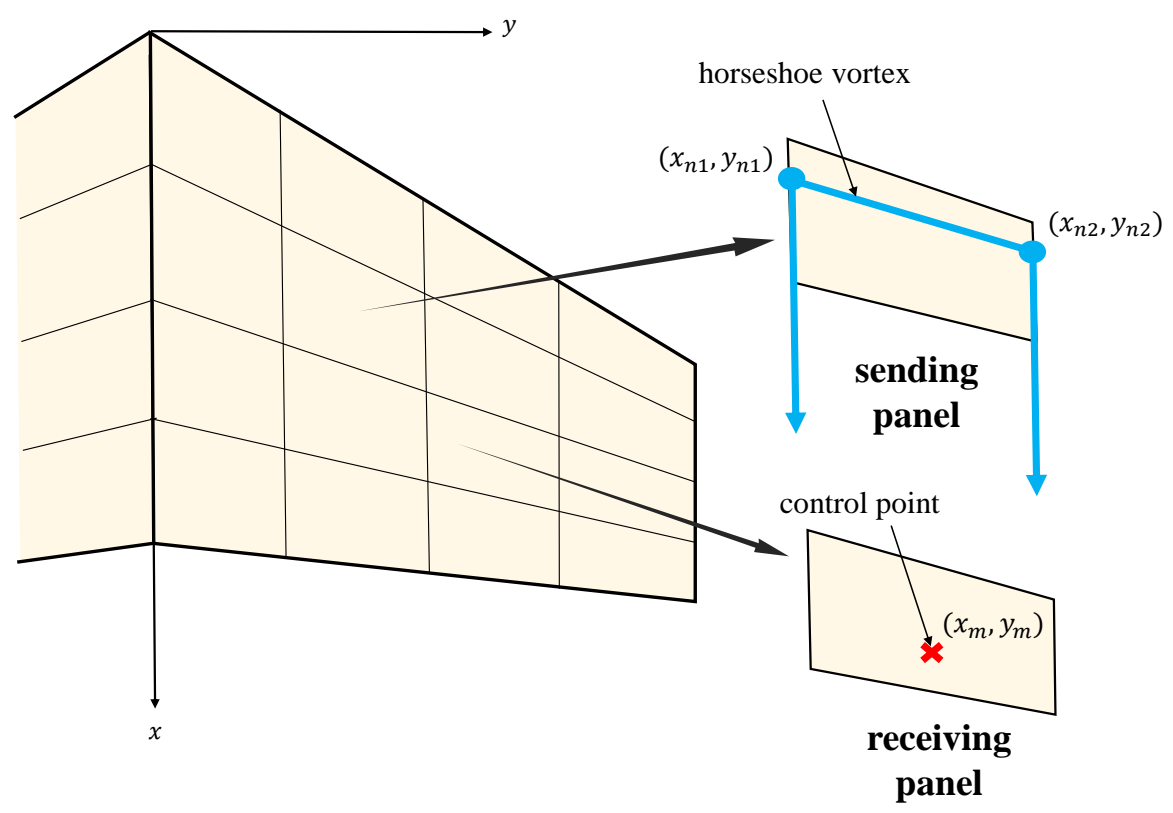

Figure 3.1: Vortex lattice method receiving and sending panels

downwash, $w_{m}$, due to the lifting surface at the $m^{t h}$ control point can be approximated for small angles of incidence, $\alpha$,

$$
w_{m}=-U_{\infty} \alpha
$$

where $U_{\infty}$ is the freestream velocity. It may be shown the control point should be located at the $3 / 4$-chord to enforce the surface tangent flow boundary condition for each panel. The downwash due to the horseshoe vortices from all $2 n$ panels at a given control point 
is calculated using the Biot-Savart law

$$
\begin{aligned}
w_{m, n}=\frac{\Gamma_{n}}{4 \pi} & \left\{\frac{1}{\left(x_{m}-x_{1 n}\right)\left(y_{m}-y_{2 n}\right)-\left(x_{m}-x_{2 n}\right)\left(y_{m}-y_{1 n}\right)}\right. \\
& {\left[\frac{\left(x_{2 n}-x_{1 n}\right)\left(x_{m}-x_{1 n}\right)+\left(y_{2 n}-y_{1 n}\right)\left(y_{m}-y_{1 n}\right)}{\sqrt{\left(x_{m}-x_{1 n}\right)^{2}+\left(y_{m}-y_{1 n}\right)^{2}}}\right.} \\
& \left.-\frac{\left(x_{2 n}-x_{1 n}\right)\left(x_{m}-x_{2 n}\right)+\left(y_{2 n}-y_{1 n}\right)\left(y_{m}-y_{2 n}\right)}{\sqrt{\left(x_{m}-x_{2 n}\right)^{2}+\left(y_{m}-y_{2 n}\right)^{2}}}\right] \\
& +\frac{1}{y_{1 n}-y_{m}}\left[1+\frac{x_{m}-x_{1 n}}{\sqrt{\left(x_{m}-x_{1 n}\right)^{2}+\left(y_{m}-y_{1 n}\right)^{2}}}\right] \\
& \left.-\frac{1}{y_{2 n}-y_{m}}\left[1+\frac{x_{m}-x_{2 n}}{\sqrt{\left(x_{m}-x_{2 n}\right)^{2}+\left(y_{m}-y_{2 n}\right)^{2}}}\right]\right\}
\end{aligned}
$$

where $\Gamma_{n}$ is the vortex strength of the $n^{\text {th }}$ panel. A system of linear algebraic equations relating the downwash of the $m^{\text {th }}$ control point due to the $2 n$ horseshoe vortices with the downwash from the freestream is established by enforcing the tangent flow boundary condition [23]

$$
\left[A_{m n}\right]\{\Gamma\}+U_{\infty}\{\alpha\}=0
$$

where $\left[A_{m n}\right]$ is the influence coefficient matrix. For a wing with a defined geometry and angle of attack, the vortex distribution may be found. The lift of each panel is calculated with the Kutta-Joukowski theorem

$$
\{L\}=\rho_{\infty} U_{\infty} \Delta y_{a}\{\Gamma\}
$$

where $\rho_{\infty}$ is the freestream density and $\Delta y_{a}$ is the width of each panel. The lift distribution isn't directly calculated in an aeroelastic analysis. Rather, the influence coefficient matrix is used to relate the aerodynamic loading to the structural deflection. 


\subsection{Unsteady Aerodynamics}

Many principal aeroelastic phenomena (flutter, gust response, etc.) are unsteady problems such that the accurate modeling of unsteady aerodynamic loads is paramount. The challenge of modeling aerodynamic forces on an unsteady wing may seem daunting because of the complexity associated with changes in circulation and wake, however, this task is simplified when considering only the flutter phenomenon. In a flutter analysis, the lifting surface is assumed to undergo oscillatory harmonic motion at the outset, which reduces the overall complexity of the unsteady aerodynamics [24]. The harmonic assumption leads the aerodynamic problem to the frequency-domain because there is only a single oscillatory frequency of interest.

Unsteady panel methods modeling the forces on harmonically oscillating wings are then well-suited for aeroelastic flutter analysis. The Doublet Lattice Method (DLM) has been the dominant unsteady oscillatory panel method used by the aeronautical industry since its publication in 1969. Alternatively, the Doublet Point Method (DPM) is another popular unsteady panel method used by aeroelasticians. Both of these methods are explored in the present section.

\subsubsection{Doublet Lattice Method}

The doublet lattice method of Albano and Rodden [25] is an extension of the vortex lattice method for oscillating surfaces. It is based on linearized potential flow theory and is readily applicable to a wide-range of wing configurations. Similar to the VLM, the lifting surface is discretized into a series of trapezoidal lifting panels of number $i$. Acceleration potential doublets of constant strength are fixed at the 1/4-chord line of each panel as shown in Figure 3.2. The normalwash at the collocation point due to the

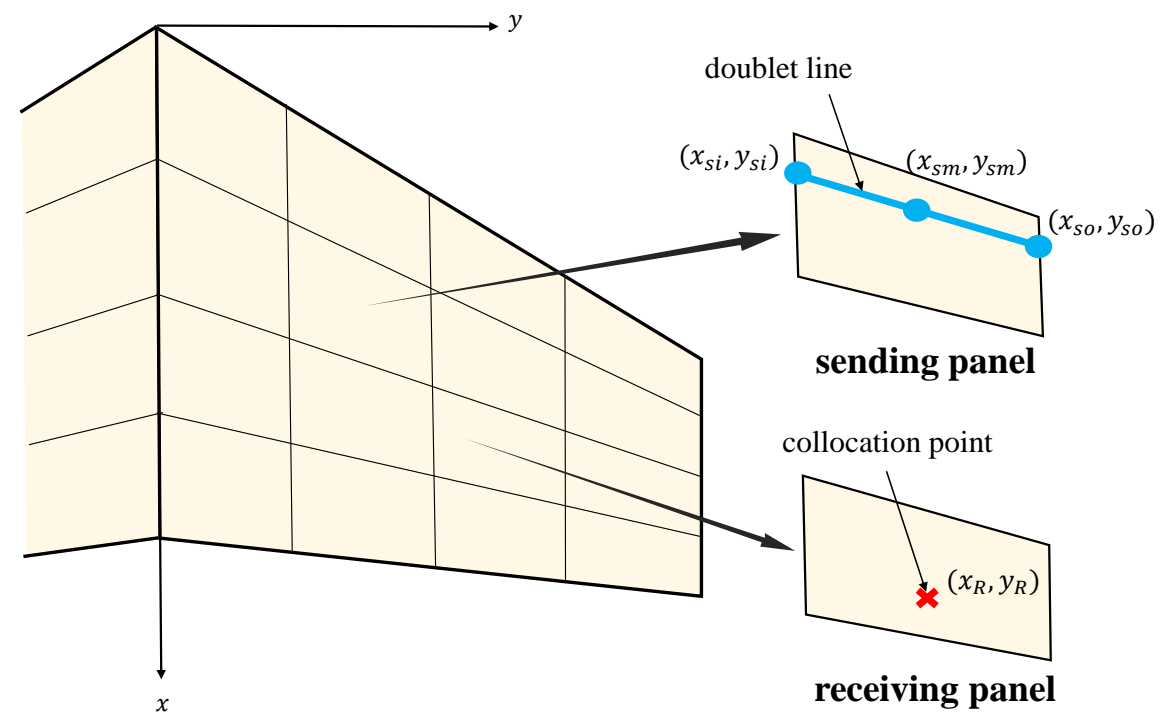

Figure 3.2: Doublet lattice method receiving and sending panels 
doublet lines is related to the panel pressure coefficient and kernel function for a planar configuration by

$$
\bar{w}_{i}=\frac{1}{8 \pi} \iint_{A} \Delta C_{p}(\xi, \eta) K((x-\xi),(y-\eta), \omega, M) d \xi d \eta
$$

where $\bar{w}$ is the normalwash nondimensionalized with the freestream velocity, $C_{p}$, is the panel pressure coefficient, $K$ is the kernel function, $\xi$ and $\eta$ are doublet midpoint coordinates, $\omega$ is the oscillating frequency, and $M$ is the Mach number. The $3 / 4$-chord location is used as the collocation point, although this is not a necessity as it is in the VLM. The system is discretized and the pressure coefficients are related to the lift per unit length along the doublet lines. A system of linear equations are established that relate the $i^{\text {th }}$ panel's normalwash with the $j^{\text {th }}$ panel's pressure coefficient through the influence coefficients, $D_{i j}$,

$$
\left\{\bar{w}_{i}\right\}=\left[D_{i j}\right]\left\{C_{p}\right\}
$$

where the influence coefficients are given by

$$
D_{i j}=\frac{1}{8 \pi} \Delta x_{j} \cos \left(\lambda_{j}\right) \int_{l_{1}}^{l_{2}} K\left(x_{0}, y_{0}, k, M\right) d l
$$

Here, $l$ is the doublet line, $\lambda$ is the doublet line sweep angle, $\Delta x$ is the average panel chord, and $k$ is the reduced frequency, which is an important aeroelastic parameter defined as

$$
k=\frac{\omega b}{U_{\infty}}
$$

with $b$ being the wing half-chord. The following delta coordinates are the difference between the "receiving" location (collocation point) and the "sending" location, which could be any of points defined along the doublet line in Figure 3.2.

$$
\begin{aligned}
& x_{0}=x_{R}-x_{s} \\
& y_{0}=y_{R}-y_{s}
\end{aligned}
$$


The kernel function for a non-planar surface is

$$
K=e^{-i k x_{0} / b} \frac{\left(K_{1} T_{1}+K_{2} T_{2}\right)}{r_{1}^{2}}
$$

For a planar surface, $T_{1}=1, T_{2}=0$, and $r_{1}=\left|y_{0}\right|$. The working form of the kernel function, $\kappa$, is the numerator of the kernel function, which may be simplified to the form

$$
\kappa=e^{-i k x_{0} / b} K_{1}
$$

where $K_{1}$ is defined as

$$
K_{1}=I_{1}+\left[\frac{M r_{1}}{R}\right]\left[\frac{e^{-i k_{1} u_{1}}}{\sqrt{1+u_{1}^{2}}}\right]
$$

The various symbols in $K_{1}$ are defined as follows:

$$
\begin{aligned}
R & =\sqrt{x_{0}^{2}+\beta^{2} r_{1}^{2}} & I_{1} & =\int_{u_{1}}^{\infty} \frac{e^{-i k_{1} u}}{\left(1+u^{2}\right)^{3 / 2}} d u \\
\beta & =\sqrt{1-M^{2}} & u_{1} & =\frac{\left(M R-x_{0}\right)}{\beta^{2} r_{1}} \\
k_{1} & =\frac{r_{1} k}{b} & &
\end{aligned}
$$

The $I_{1}$ integral remains to be evaluated. Integrating by parts results in the following expression

$$
I_{1}\left(u_{1}, k_{1}\right)=e^{-i k_{1} u_{1}}\left[1-\frac{u_{1}}{\sqrt{1+u_{1}^{2}}}-i k_{1} I_{0}\left(u_{1}, k_{1}\right)\right]
$$

with

$$
I_{0}\left(u_{1}, k_{1}\right)=e^{i k_{1} u_{1}} \int_{u_{1}}^{\infty}\left[1-\frac{u}{\sqrt{1+u^{2}}}\right] e^{-i k_{1} u} d u
$$

Laschka [26] provided a convenient and accurate approximation of $I_{0}$ for when $u_{1}>0$.

$$
I_{0}\left(u_{1}, k_{1}\right) \approx \sum_{n=1}^{11} \frac{a_{n} e^{-n c u_{1}}}{n^{2} c^{2}+k_{1}^{2}}\left(n c-i k_{1}\right)
$$


where $c=0.372$ and the $a_{n}$ coefficients for the eleven-term series are listed in Table 3.1.

Table 3.1: Laschka coefficients for approximation of $I_{0}$

\begin{tabular}{c|r}
$n$ & $a_{n}$ coefficients \\
\hline 1 & 0.24186198 \\
2 & -2.7918027 \\
3 & 24.991079 \\
4 & -111.59196 \\
5 & 271.43549 \\
6 & -305.75288 \\
7 & -41.183630 \\
8 & 545.98537 \\
9 & -644.78155 \\
10 & 328.72755 \\
11 & -64.279511
\end{tabular}

The $I_{1}$ integral is evaluated for the case $u_{1}<0$ by making use of its symmetry, where the real and imaginary components are calculated separately [27].

$$
I_{1}\left(u_{1}, k_{1}\right)=2 \operatorname{Re}\left[I_{1}\left(0, k_{1}\right)\right]-\operatorname{Re}\left[I_{1}\left(-u_{1}, k_{1}\right)\right]+i \operatorname{Im}\left[I_{1}\left(-u_{1}, k_{1}\right)\right]
$$

A parabolic approximation is made for the kernel integral in the influence coefficients of Eq. (3.7)

$$
D_{i j}=\frac{1}{8 \pi} \Delta x_{j} \cos \left(\lambda_{j}\right)\left[\left(\frac{2 e}{\eta_{0}^{2}-e^{2}}\right)\left(\eta_{0}^{2} A+\eta_{0} B+C\right)+\left(\frac{1}{2} B+\eta_{0} A\right) \ln \left(\frac{\eta_{0}-e}{\eta_{0}+e}\right)^{2}+2 e A\right]
$$

where $\eta_{0}$ is the $y_{0}$ associated with the middle sending point $\left(\eta_{0}=y_{R}-y_{s m}\right)$ and $e$ is the panel half-span.

$$
e=\frac{1}{2} l_{j} \cos \left(\lambda_{j}\right)
$$

The $A, B$, and $C$ symbols are

$$
A=\frac{\kappa_{i}-2 \kappa_{m}+\kappa_{o}}{2 e^{2}} \quad B=\frac{\kappa_{o}-\kappa_{i}}{2 e} \quad C=\kappa_{m}
$$


The subscripts of the working kernel functions represent the sending points used to define the delta coordinates, $x_{0}, y_{0}$, in each respective kernel function.

If the DLM code is only considering a semi-span, additional handling must be done to consider the effects of the entire wing span. This may be accomplished by adding the influence coefficients due to the symmetric semi-span to the existing semi-span influence coefficients. The influence coefficients due to the symmetric panels are calculated in the same manner, except for the sending points in the $y_{0}$ delta coordinate given from the symmetric panels.

In order for the DLM to produce the results of the VLM at steady state and improve the kernel integral approximation, Albano and Rodden [25] suggested subtracting the steady component, $\kappa_{s}$, from the working kernel function

$$
\kappa^{\prime}=\kappa-\kappa_{s}
$$

where as $\omega \rightarrow 0, \kappa$ becomes

$$
\kappa_{s}=1+\frac{x_{0}}{R}
$$

for the planar configuration. This creates incremental influence coefficients, $D_{i j}^{\prime}$. The effect of an VLM horseshoe vortex should then be added such that the steady component of the kernel function is integrated exactly. This results in the improved and final form of the doublet lattice influence coefficients, $\hat{D}_{i j}$

$$
\left[\hat{D}_{i j}\right]=\left[D_{i j}^{\prime}\right]+\frac{1}{2}\left[\Delta x_{a}\right]\left[A_{m n}\right]
$$

The non-dimensional normalwash due to the panel structural deformation at the collocation point, $w$, is given by

$$
\bar{w}_{i}=\frac{\partial w}{\partial x}+\frac{i k}{b} w
$$

Ultimately, the unsteady loading along the doublet line found from the panel pressure coefficients, which are calculated using the influence coefficients and normalwash by rearranging Eq. (3.6). 


\subsubsection{Doublet Point Method}

The Doublet Point Method (DPM) is an alternative unsteady oscillatory panel method introduced by Ueda and Dowell [28] in 1982. Instead of fixing a doublet line on each panel as in the DLM, this method places a single doublet at the midspan of the panel 1/4-chord location as shown in Figure 3.3. Lift is then represented as a concentrated force at this point rather than a constant distribution across the quarter-chord. The control (or upwash) point is located at the same $3 / 4$-chord location as before. Where the DLM involved an averaging procedure to account for the singular behavior of the normalwash downstream of the doublet line, the DPM only requires an averaging of the singular contribution to the normalwash immediately behind the doublet point, which improves computational efficiency [29]. The DPM begins from the same integral equation relating

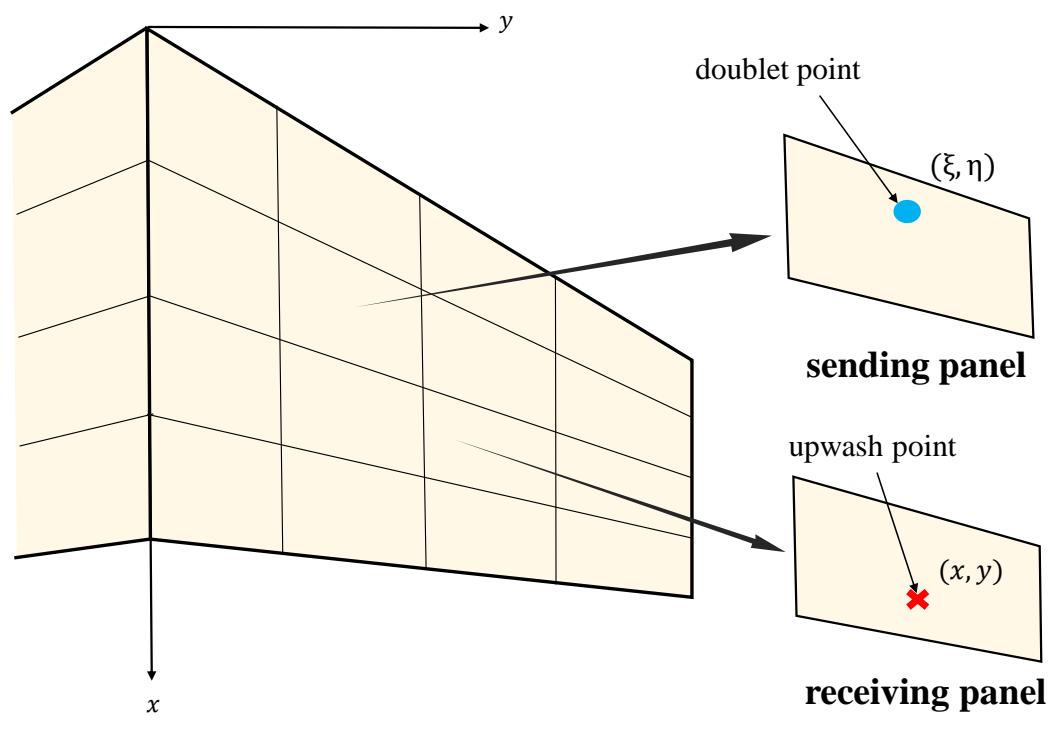

Figure 3.3: Doublet point method receiving and sending panels

the panel pressure coefficients to the kernel function as the DLM for a planar configuration

$$
\bar{w}_{i}=\frac{1}{8 \pi} \iint_{A} \Delta C_{p}(\xi, \eta) K\left(\overline{x_{0}}, \overline{y_{0}}\right) d \xi d \eta
$$

All variables in this equation are non-dimensionalized. The normalwash is non-dimensionalized as before with the freestream velocity, $U_{\infty}$, however, the lengths are non-dimensionalized by the root semi-chord, $b$. Again, the governing integral equation is discretized and a system of linear equations are formed that relate the $i^{\text {th }}$ panel's normalwash with the $j^{\text {th }}$ panel's pressure coefficient through influence coefficients, $D_{i j}$

$$
\left\{\bar{w}_{i}\right\}=\left[D_{i j}\right]\left\{C_{p}\right\}
$$


where the influence coefficients are given this time by

$$
D_{i j}=\frac{1}{8 \pi} \bar{\Delta}_{j} K\left(\bar{x}_{0}, \bar{y}_{0}\right)
$$

Here, $\Delta$ is the panel area nondimensionalized by $b^{2}$. The kernel function has the following form

$$
K\left(\overline{x_{0}}, \overline{y_{0}}\right)=e^{-i k \overline{x_{0}}}\left[\frac{M e^{i k X}}{R \sqrt{X^{2}+r^{2}}}+B(k, r, X)\right]
$$

The reduced frequency, $k$, is defined as before in the DLM. The remaining parameters in the kernel function are defined as follows:

$$
\begin{aligned}
\bar{x}_{0} & =\frac{x_{i}-\xi_{j}}{b} & R & =\sqrt{\bar{x}_{0}^{2}+\beta^{2} r^{2}} \\
\bar{y}_{0} & =\frac{y_{i}-\eta_{j}}{b} & X & =\frac{\overline{x_{0}}-M R}{\beta^{2}} \\
r & =\left|\bar{y}_{0}\right| & &
\end{aligned}
$$

The $B(k, r, X)$ function in the kernel is an integral of complex values. This integral is separated into two real functions as shown.

$$
\begin{aligned}
& B(k, r, X)=\int_{-\infty}^{X} \frac{e^{i k v}}{\left(v^{2}+r^{2}\right)^{3 / 2}} d v \\
& B(k, r, X)=B_{R}(k, r, X)+i B_{I}(k, r, X)
\end{aligned}
$$

These two real integral functions are solved by series expansion. The series have originally been written with a starting index of zero, but in the present outline, the solution to the two integral functions are written for a starting index of one. This is done for ease of implementation into MATLAB. The two functions are

$$
\begin{aligned}
B_{R}(k, r, X) & =\sum_{n=1}^{\infty}\left[(-1)^{n-1} U_{2 n-1}\right]-\frac{k^{2}}{2} \sum_{n=1}^{\infty}\left\{\frac{(k r / 2)^{2 n-1}}{n[(n-1) !]^{2}}\right. \\
& \left.\times\left[\sum_{m=1}^{n}\left(\frac{1}{m}\right)+\frac{1}{2 n}-\gamma-\ln \frac{k}{2}\right]\right\} \\
B_{I}(k, r, X) & =\sum_{n=1}^{\infty}\left[(-1)^{n-1} U_{2 n}\right]+\frac{\pi}{4} k^{2} \sum_{n=1}^{\infty}\left[\frac{(k r / 2)^{2 n-1}}{n[(n-1) !]^{2}}\right]
\end{aligned}
$$


where $\gamma$ is Euler's constant. The first three terms of the $U$ function are

$$
\begin{aligned}
U_{1} & =\frac{1}{\sqrt{X^{2}+r^{2}}\left(\sqrt{X^{2}+r^{2}}-X\right)} \\
U_{2} & =\frac{-k}{\sqrt{X^{2}+r^{2}}} \\
U_{3} & =-\frac{k^{2}}{2}\left[\frac{X}{\sqrt{X^{2}+r^{2}}}+\ln \left(\sqrt{X^{2}+r^{2}}-X\right)\right]
\end{aligned}
$$

The remaining $U$ terms are given by the recurring formula

$$
U_{n}=\frac{k}{(n-3)(n-1) !} \frac{(k X)^{n-2}}{\sqrt{X^{2}+r^{2}}}-\frac{(k r)^{2}}{\left(n^{2}-4 n+3\right)} U_{n-2} \quad(n \geq 4)
$$

This solution procedure for the $B(k, r, X)$ works well when $r \neq 0$ or when $r=0$ and $X<0$, however, additional handling is necessary to account for the singularity in the kernel when a receiving point is directly downstream of a doublet point. Through the method outlined by Ueda and Dowell [28], $B_{R}$ may be calculated for this singularity by the following, where $\bar{\sigma}$ is the non-dimensional panel half-width.

$$
\begin{array}{r}
B_{R}(k, r, X)=-B_{R}(k, 0,-X)-\frac{\pi^{2}}{6 \bar{\sigma}_{j}^{2}}+k^{2}\left(\ln \frac{k \bar{\sigma}_{j}}{2}+\gamma-\frac{3}{2}\right) \\
\left(r<\bar{\sigma}_{j}, X>0\right)
\end{array}
$$

The effect of the symmetric semi-span panels on the influence coefficients and the general solution procedure is treated in the same manner as the DLM. 


\section{Chapter 4}

\section{Aeroelaticity Models}

\subsection{Introduction}

The fundamental objective of a computational aeroelastic analysis is to accurately couple the structural and aerodynamic models. Without a reliable coupling methodology, even the most sophisticated structural and aerodynamic models can fail to produce valid aeroelasticity results. This section covers the procedure behind aeroelastic coupling and presents the solution for divergence and flutter speeds of the present aeroelastic model.

\subsection{Aeroelastic Coupling}

Aeroelastic coupling involves defining the communication between structural and aerodynamic deformation and loads. This communication is not trivial because the structural and aerodynamic meshes are often different in terms of mesh size and node positions. An example of this process is illustrated in Figure 4.1. The aerodynamic point loads, such as from the DPM, are transformed to equivalent nodal loads on the finite element structure. From these loads, the finite element model produces nodal deformations. The nodal deformations are then transformed by means of interpolation to aerodynamic control points, which are used to calculate updated aerodynamic loads. These transformations are commonly performed by using one of the following methodologies:

1. Surface spline method

2. Finite element shape function method

The surface (or infinite) spline method has been developed by Harder and Desmarais [30] and is widely used in aeroelasticity. It involves defining different grids sets and performing the transformation through interpolation matrices. The method is derived from infinite 


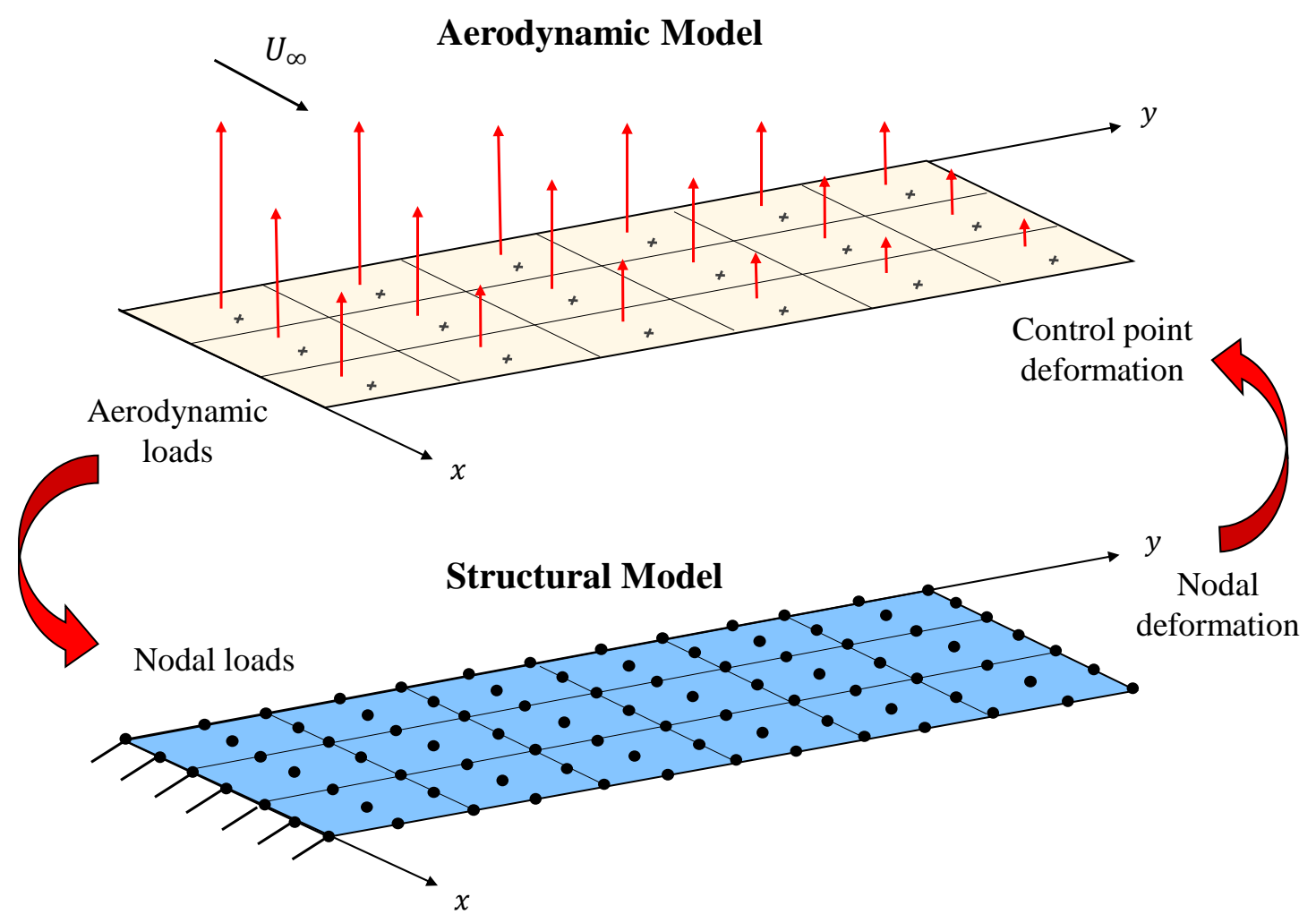

Figure 4.1: Aeroelasticity coupling illustration

plate theory with the advantage that the point coordinates do not need to be included in a rectangular array and that slopes may be found by simply differentiating the function.

Alternatively, the finite element shape function method involves performing the numerical integration and interpolation with the existing finite element shape functions, as demonstrated by Mahran et al. [13]. This method is advantageous because the finite element shape functions have already been developed and are easily employed.

\section{Finite Element Shape Function Transformation}

The aim is to formulate transformation matrices with finite element shape functions that will interpolate the structural deformation to the control point deformation and integrate the aerodynamic loads to the structural nodes. The deformation transformation is first developed. Consider the interpolation of a panel normalwash, $\bar{w}$, at a single point from the element deformation vector, $\left\{q_{e}\right\}$, such as in the DLM:

$$
\bar{w}=\left(\frac{i k}{b}\lfloor H\rfloor+\left\lfloor\frac{\partial H}{\partial x}\right\rfloor\right)\left\{q_{e}\right\}
$$

Here, $\lfloor H\rfloor$ is the set of Hermite shape functions evaluated at the control point in terms of $\xi$ and $\eta$. The deformation transformation matrix, $\left[T_{1}\right]$, consists of rows of these 
interpolation expressions for every control point present within an element and has the form

$$
\left[T_{1}\right]=\left[\begin{array}{c}
\frac{i k}{b}\lfloor H\rfloor+\left\lfloor\frac{\partial H}{\partial x}\right\rfloor \\
\vdots
\end{array}\right]
$$

The transformation is of size $\left(N_{a} \times D O F_{e}\right)$, where $N_{a}$ is the number of aerodynamic panels per element and $D O F_{e}$ is the element degree of freedom number. The normalwash vector is then directly related to the element structural deformation as follows.

$$
\{\bar{w}\}=\left[T_{1}\right]\left\{q_{e}\right\}
$$

This is done identically in the DPM. The load transformation matrix, however, will differ in appearance depending on the aerodynamic model being used. Consider the relationship between the finite element nodal loads, $\left\{Q_{e}\right\}$ and the aerodynamic load, $L$, acting as a constant line load distribution across a panel $1 / 4$-chord as in the DLM

$$
\left\{Q_{e}\right\}=\left(\frac{\Delta y_{s}}{2} \int\lfloor H\rfloor^{T} d \xi\right) \frac{L}{\Delta y_{a}}
$$

Here, $\Delta y_{s}$ and $\Delta y_{a}$ are the widths of the structural and aerodynamic elements, respectively, $\lfloor H\rfloor$ is the set of Hermite shape functions evaluated at the $\eta$ value associated with the panel quarter chord, and the integral acts over the $\Delta \xi$ associated with the panel doublet line. The load transformation matrix, $\left[T_{2}\right]$, consists of columns of these integral expressions for every aerodynamic panel within the element and has the form

$$
\left[T_{2}\right]=\frac{1}{\Delta y_{a}}\left[\frac{\Delta y_{s}}{2} \int\lfloor H\rfloor^{T} d \xi \quad \cdots\right]
$$

where the matrix is of size $\left(D O F_{e} \times N_{a}\right)$. This transformation directly relates the vector of aerodynamic loads to the equivalent finite element nodal loads as follows.

$$
\left\{Q_{e}\right\}=\left[T_{2}\right]\left\{L_{e}\right\}
$$

The load transformation will have a much simpler appearance for the DPM, as no integration is involved because there are only point loads. In this case, $\left[T_{2}\right]$, simply 
consists of shape functions evaluated at the doublet points.

\subsection{Divergence}

Divergence occurs when the moment acting on the wing due to aerodynamic loading overcomes the restorative moment due to its structural stiffness, causing structural failure. The speed of the freestream air at which divergence occurs is called the divergence speed. Although aircraft flutter is usually of greater concern, divergence analysis remains necessary and characterizes the stiffness of the wing [2].

Furthermore, divergence analysis becomes even more important when considering composite wings, as it is well-known that specific ply orientation and layups can cause the wing to be more vulnerable to divergence than to flutter. Therefore, accurate prediction of divergence speeds are required for the aeroelastic optimization of a composite wing.

In the context of computational aeroelasticity, the divergence speeds are typically found by constructing an instability eigenvalue problem relating the finite element stiffness with an Aerodynamic Influence Coefficient (AIC) matrix. The AIC matrix relates the aerodynamic loading to the dynamic pressure and panel deformations. The following divergence analysis is formulated using the vortex lattice method, however, applying the doublet lattice method with $k=0$ is equivalent. Note there is no requirement for an initial angle of attack to be specified in this analysis.

First, consider that the finite element plate global stiffness, $[K]$, is related to the global load vector, $\{Q\}$, through the global displacements by

$$
[K]\{q\}=\{Q\}
$$

The global displacement vector is related to the aerodynamic panel angles of attack, $\{\alpha\}$, through the global deformation transformation

$$
\{\alpha\}=\left[T_{1}\right]\{q\}
$$

The global load vector is related to the aerodynamic lift vector through the global load transformation

$$
\{Q\}=\left[T_{2}\right]\{L\}
$$


The aerodynamic lift vector may be written out in terms of its influence coefficients from Eqs. (3.3) and (3.4) of the VLM.

$$
\{L\}=-\rho_{\infty} U_{\infty}^{2} \Delta y_{a}\left[A_{m n}\right]^{-1}\{\alpha\}
$$

Substituting Eqs. (4.8) and (4.10) into Eq. (4.9), the global load vector may be updated to have the form

$$
\{Q\}=\frac{1}{2} \rho_{\infty} U_{\infty}\left(-2 \Delta y_{a}\left[T_{2}\right]\left[A_{m n}^{-1}\right]\left[T_{1}\right]\right)\{q\}
$$

Introducing the dynamic pressure, $q_{\infty}$, the expression simplifies to

$$
\{Q\}=q_{\infty}\left[A_{d}\right]\{q\}
$$

where $\left[A_{d}\right]$ is the divergence aerodynamic influence coefficient matrix that is summarized as

$$
\left[A_{d}\right]=\left(-2 \Delta y_{a}\left[T_{2}\right]\left[A_{m n}\right]^{-1}\left[T_{1}\right]\right)
$$

The divergence dynamic pressure is calculated by solving the eigenvalue problem created by combining Eqs. (4.7) and (4.12)

$$
\left([K]-q_{\infty}\left[A_{d}\right]\right)\{q\}=\{0\}
$$

The solution to this static equation is the dynamic pressure at which the system is unstable. The divergence speed is simply calculated from the dynamic pressure by

$$
U_{\infty}=\sqrt{\frac{2 q_{\infty}}{\rho_{\infty}}}
$$

The air density is assumed to be at standard sea level. 


\subsection{Flutter}

Flutter is considered to be the most critical aeroleastic phenomena. As defined previously, flutter occurs at a certain air speed when energy is effectively extracted from the air stream to produce sustained structural oscillation. This freestream speed is called the flutter speed, and it is desirable to increase this speed to the highest value possible so as to avoid structural failure. Aeroelastic tailoring with composite materials involves utilizing ply layup and orientation to increase flutter speed.

The solution to flutter speeds is not as simple as that for divergence. This is because the oscillating frequency is an unknown within the flutter analysis, whereas it is known to be zero in the divergence analysis. Thus, the solution requires an iterative approach known as 'frequency matching' [2]. Nevertheless, an eigenvalue problem must still be established relating the finite element stiffness to the flutter aerodynamic influence coefficients.

First, consider the plate equation of motion in the absence of damping, where $[M]$ is the global inertia matrix.

$$
[M]\{\ddot{q}\}+[K]\{q\}=\{Q\}
$$

Harmonic oscillation of the structure is assumed at the outset in a flutter analysis. Assuming $\{q\}=\left\{q_{0}\right\} e^{i \omega t}$, the equation simplifies to

$$
\left(-\omega^{2}[M]+[K]\right)\{q\}=\{Q\}
$$

Now, let $\left[D_{i j}\right]$ represent the general influence coefficients for either the DLM or the DPM. From either of these methods, the panel pressure coefficient is given by

$$
\left\{C_{p}\right\}=\left[D_{i j}\right]^{-1}\left\{\bar{w}_{i}\right\}
$$

The definition of the pressure coefficient follows, with $\Delta P$ as the pressure difference between the top and bottom of the panel

$$
C_{p}=\frac{\Delta P}{\frac{1}{2} \rho_{\infty} U_{\infty}^{2}}
$$


Assuming that the pressure difference is given by the aerodynamic load divided by the panel area, the complex aerodynamic load vector is given by Eqs. (4.18) and (4.19)

$$
\{L\}=\frac{1}{2} \rho_{\infty} U_{\infty}^{2} \Delta x_{a} \Delta y_{a}\left[D_{i j}\right]^{-1}\left\{\bar{w}_{i}\right\}
$$

Similar to the divergence analysis, the normalwash is related to the global displacements through the deformation transformation and the global load vector is related to the complex aerodynamic lift vector through the load transformation as follows.

$$
\begin{aligned}
& \left\{\bar{w}_{i}\right\}=\left[T_{1}\right]\{q\} \\
& \{Q\}=\left[T_{2}\right]\{L\}
\end{aligned}
$$

The global load vector is updated by substituting Eq. (4.21) into Eq. (4.20)

$$
\{Q\}=q_{\infty}\left(\Delta x_{a} \Delta y_{a}\left[T_{2}\right]\left[D_{i j}\right]^{-1}\left[T_{1}\right]\right)\{q\}
$$

The flutter AIC matrix is summarized as

$$
\left[A_{f}\right]=\Delta x_{a} \Delta y_{a}\left[T_{2}\right]\left[D_{i j}\right]^{-1}\left[T_{1}\right]
$$

By substituting the global load vector from Eq. (4.22) into Eq. (4.17), the equation of motion becomes

$$
\left(-\omega^{2}[M]+[K]-q_{\infty}\left[A_{f}\right]\right)\{q\}=\{0\}
$$

At this point in the formulation, structural damping and the solution procedure must be considered. There are two well-established solution methodologies associated with flutter: the $k$-method and $p$ - $k$ method. While the $p$ - $k$ method is more rigorous and provides more accurate damping trends, the $k$ method is simpler, easier to implement, and predicts the same exact flutter speeds as the $p$ - $k$ method [2].

The $k$-method solution begins by introducing an artificial structural damping term, $[C]$,

$$
[C]=i g[K]
$$


where $g$ is the structural damping coefficient. The damping matrix is added to the equation of motion and the freestream velocity within the dynamic pressure is written in terms of the reduced frequency using Eq. (3.8).

$$
\left([M]+\frac{1}{2} \rho_{\infty}\left(\frac{b}{k}\right)^{2}\left[A_{f}\right]-\left(\frac{i g+1}{\omega^{2}}\right)[K]\right)\{q\}=\{0\}
$$

Denoting the sum of the inertia matrix and aerodynamic influence matrix terms as $[F]$, the eigenvalue problem becomes

$$
\left([F]-\left(\frac{i g+1}{\omega^{2}}\right)[K]\right)\{q\}=\{0\}
$$

If the eigenvalue is represented by $\lambda$, then the frequency and structural damping coefficient may be found for various modes at a given reduced frequency from

$$
\omega=\frac{1}{\sqrt{\operatorname{Re}(\lambda)}} \quad g=\frac{\operatorname{Im}(\lambda)}{\operatorname{Re}(\lambda)}
$$

The $k$-method solution then involves iteratively analyzing the eigenvalue problem and calculating the freestream velocity for a range of reduced frequencies until one of the mode's damping coefficients becomes zero, where flutter occurs. The damping and natural frequency trends are typically plotted against freestream speed for a range of reduced frequencies in $V g$ and $V \omega$ plots. These plots and the general solution procedure are explored in more detail later. 


\section{Chapter 5}

\section{Numerical Verification}

The structural, aerodynamic, and aeroelasticity models described thus far are all written as codes in MATLAB. It is necessary to first individually validate or verify these codes before performing computational experiments. This is accomplished by comparing results with existing experimental or computational results available in literature. The present section documents the verification of each model.

\subsection{Structural Model Verification}

The structural model verification involves the verification of the finite element codes associated with the new MONNA element, the Melosh element, and the BFS element. However, emphasis will be placed on the MONNA element, as it is untested. All three plate finite elements have been modeled with the present HSDT and coded in MATLAB. The necessary verification of all three elements will offer a direct comparison of the performance among non-conforming, conforming, and higher-order conforming rectangular $C^{1}$-continuous plate elements.

\subsubsection{Isotropic Plates}

There are well-established analytical solutions for the deflection of simply supported isotropic plates such as the Naiver Solution. The boundary conditions for the present HSDT plate simply supported on all sides is shown in Figure 5.1. A square isotropic plate $(E=70 \mathrm{GPa}, \nu=0.25)$ with dimensions of $(5 \times 5 \times 0.01) \mathrm{m}$ subjected to a uniformly distributed load of $10 \mathrm{~Pa}$ is analyzed and compared in Table 5.1 for each structural finite element model. There is good agreement among all HSDT finite element models and the analytical Naiver solution.

It is understood that accurate calculation of structural natural frequencies is a prerequisite for flutter analysis [7]. To this end, the natural frequencies of an isotropic square plate are compared with Naiver solution results provided by Bogner, Fox, and 


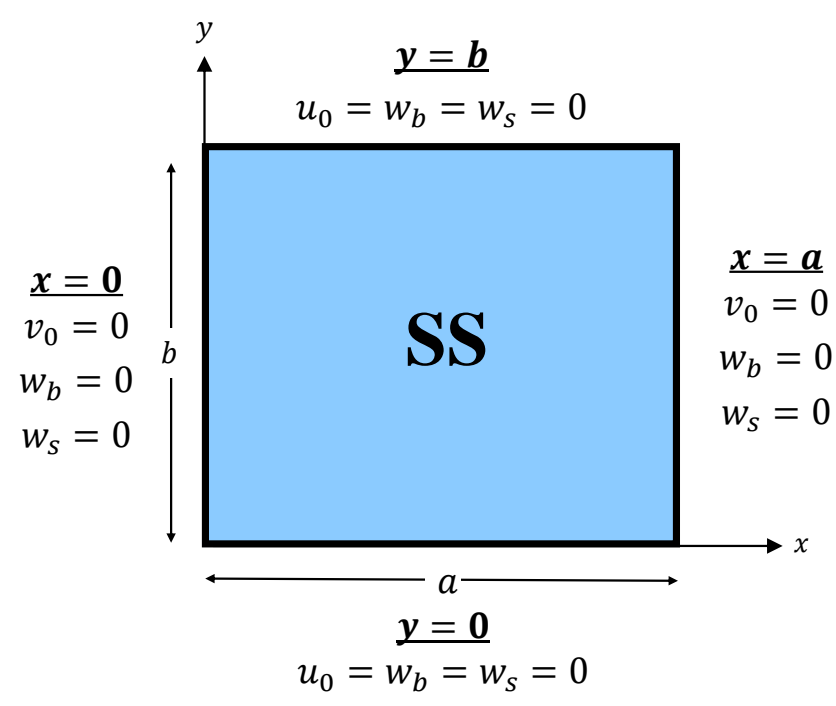

Figure 5.1: FEM boundary conditions for plate simply supported on all edges

Table 5.1: Maximum deflection and slope of square isotropic plate

\begin{tabular}{lcccc}
\hline Model & Type & Elements & Deflection $(\mathrm{m})$ & Slope \\
\hline Naiver Solution & Analytical & - & 0.004081 & 0.002707 \\
MONNA Element & FEM & $4 \times 4$ & 0.004081 & 0.002708 \\
BFS Element & FEM & $10 \times 10$ & 0.004081 & 0.002708 \\
Melosh Element & FEM & $24 \times 24$ & 0.004088 & 0.002692 \\
\hline
\end{tabular}

Schmidt [22] in Table 5.2. The square plate $\left(E=30\right.$ Msi, $\nu=0.3, \rho=0.001$ slugs $/$ in. $\left.{ }^{3}\right)$ is simply supported with dimensions of $(10 \times 10 \times 1)$ in. There is also good agreement

Table 5.2: Natural frequencies of square isotropic plate $(\mathrm{rad} / \mathrm{s})$

\begin{tabular}{lcccccc}
\hline Model & Type & Elements & $\omega_{1}$ & $\omega_{2}$ & $\omega_{3}$ & $\omega_{4}$ \\
\hline Naiver Solution [22] & Analytical & - & 1035 & 2587 & 4138 & 5173 \\
MONNA Element & FEM & $4 \times 4$ & 1034 & 2584 & 4133 & 5167 \\
BFS Element & FEM & $10 \times 10$ & 1034 & 2584 & 4132 & 5166 \\
Melosh Element & FEM & $18 \times 18$ & 1033 & 2577 & 4106 & 5149 \\
\hline
\end{tabular}

between the HSDT finite element models and the Naiver solution for natural frequencies. The small difference in the first few natural frequencies between the finite element results and the analytical solution is likely due to the analytical solution not accounting for shear deformation. 


\subsubsection{Composite Plates}

Reddy [18] has documented an extensive collection of data comparing the analytical and finite element results of composite plates with CLPT, FSDT, and HSDT. The nondimensional defection and natural frequency results are used here to verify the present finite element composite plate codes. A square plate is analyzed with simply supported boundary conditions as displayed in Figure 5.1. The loading is sinusoidal for all cases and is defined by

$$
Q(x, y)=Q_{0} \sin \left(\frac{\pi x}{a}\right) \sin \left(\frac{\pi y}{b}\right)
$$

The nondimensional center deflections, $\bar{w}$, of square cross-ply composite plates are compared in Table 5.3, where the layup is $[0 / 90]_{5}$ and side-to-thickness ratio is $b / t=5$. The material properties are representative of graphite-epoxy with $E_{1}=25 E_{2}$, $G_{12}=G_{13}=0.5 E_{2}, G_{23}=0.2 E_{2}$, and $\nu_{12}=0.25$. The center deflection is nondimensionalized by

$$
\bar{w}=w_{\max } \frac{E_{2} t^{3}}{Q_{0} b^{4}}(100)
$$

Table 5.3: Nondimensional deflection of square $[0 / 90]_{5}$ composite plate

\begin{tabular}{lcccc}
\hline Model & Type & Elements & FSDT & HSDT \\
\hline Reddy [18] & Analytical & - & 1.137 & 1.129 \\
Reddy [18] & FEM & $4 \times 4$ & 1.137 & 1.135 \\
MONNA Element & FEM & $4 \times 4$ & 1.137 & 1.130 \\
BFS Element & FEM & $4 \times 4$ & 1.137 & 1.129 \\
Melosh Element & FEM & $12 \times 12$ & 1.141 & 1.134 \\
\hline
\end{tabular}

The center deflections for all HSDT elements are in good agreement with existing analytical and finite element results. It must be noted the relative accuracy of each finite element model in Table 5.3 depends on the layup and length-to-thickness ratio. This specific verification test is not necessarily indicative of each element's general performance.

The nondimensional fundamental natural frequencies, $\bar{\omega}$, of a simply supported composite plate $\left([0 / 90]_{5}, b / t=5\right)$ are compared in Table 5.4. Here, the material properties are $E_{1}=40 E_{2}, G_{12}=G_{13}=0.6 E_{2}, G_{23}=0.5 E_{2}$, and $\nu_{12}=0.25$. The natural 
frequencies are nondimensionalized by

$$
\bar{\omega}=\omega\left(\frac{b^{2}}{t}\right) \sqrt{\frac{\rho}{E_{2}}}
$$

Table 5.4: Nondimensional fundamental natural frequency of square $[0 / 90]_{5}$ composite plate

\begin{tabular}{lcccc}
\hline Model & Type & Elements & FSDT & HSDT \\
\hline Reddy [18] & Analytical & - & 11.644 & 11.673 \\
Reddy [18] & FEM & $4 \times 4$ & 11.647 & 11.664 \\
MONNA Element & FEM & $4 \times 4$ & 11.644 & 11.673 \\
BFS Element & FEM & $4 \times 4$ & 11.649 & 11.679 \\
Melosh Element & FEM & $12 \times 12$ & 11.619 & 11.647 \\
\hline
\end{tabular}

The natural frequencies for all HSDT elements are in good agreement with existing analytical and finite element results. Again, this specific verification test is not necessarily indicative of each elements general performance, however, the results from Tables 5.3 and 5.4 demonstrates the competency and accuracy of the untested MONNA element. The results also demonstrate the convergence issues associated with non-conforming elements, as the Melosh element is relatively inaccurate even with a higher number of elements. 


\subsection{Aerodynamic Model Verification}

The aerodynamic model verification involves comparing the results of each potential flow panel method code with those presented in literature. Unlike the structural model, these methods are being directly reproduced such that the results should match exactly.

\subsubsection{Vortex Lattice Method}

First, the steady vortex lattice method code is considered. The code is verified by comparing the nondimensional vortex strengths of a $45^{\circ}$ swept constant-chord wing calculated by Bertin and Cummings [23]. The half-span is discretized into four spanwise elements as shown in Figure 5.2.

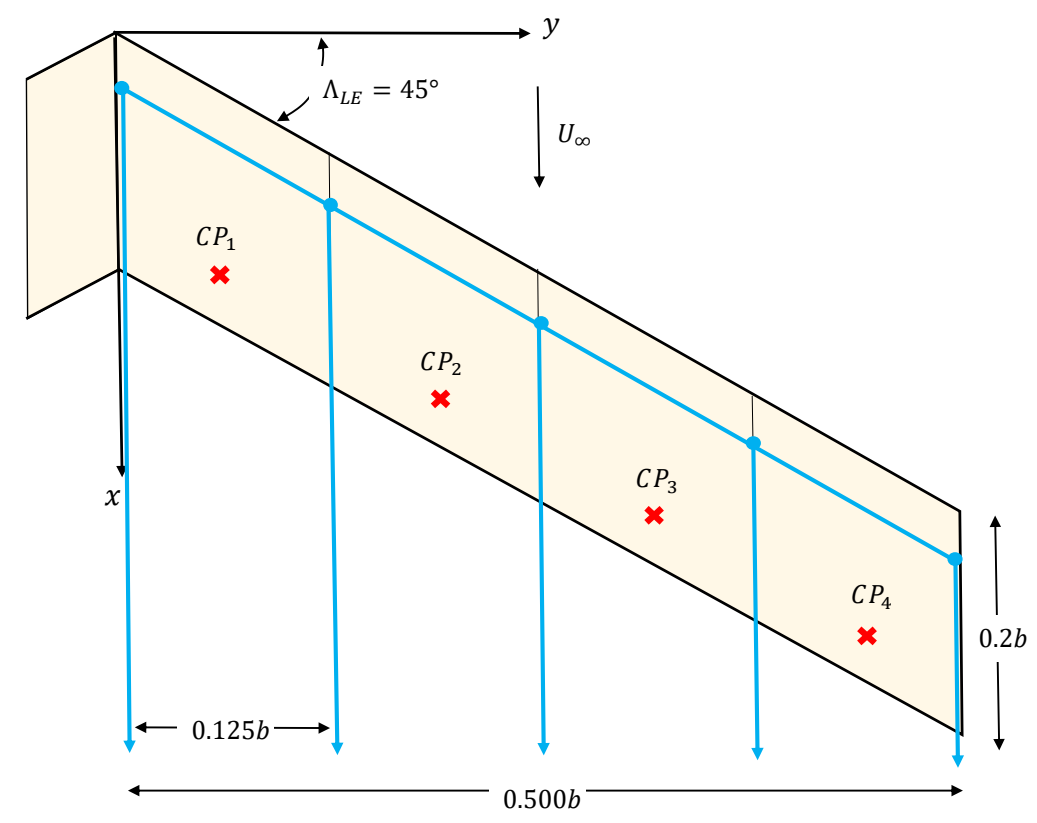

Figure 5.2: Vortex lattice method verification configuration [23]

The calculated vortex strengths and overall wing lift coefficient are presented in Table 5.5. The results match exactly with those produced by Bertin and Cummings.

Table 5.5: Nondimensional vortex strengths and lift coefficient using VLM

\begin{tabular}{cc}
\hline Parameter & Present \\
\hline$\Gamma_{1}$ & $0.0273\left(4 \pi b U_{\infty} \alpha\right)$ \\
$\Gamma_{2}$ & $0.0287\left(4 \pi b U_{\infty} \alpha\right)$ \\
$\Gamma_{3}$ & $0.0286\left(4 \pi b U_{\infty} \alpha\right)$ \\
$\Gamma_{4}$ & $0.0250\left(4 \pi b U_{\infty} \alpha\right)$ \\
$C_{L}$ & $1.096 \pi \alpha$ \\
\hline
\end{tabular}




\subsubsection{Doublet Lattice Method}

The doublet lattice method code is verified with Blair [27] by comparing complex pressure coefficients of a wing undergoing heave oscillation of unit amplitude. The half-span is of size $(12 \times 12) \mathrm{m}$ and is discretized into nine panels as shown in Figure 5.3

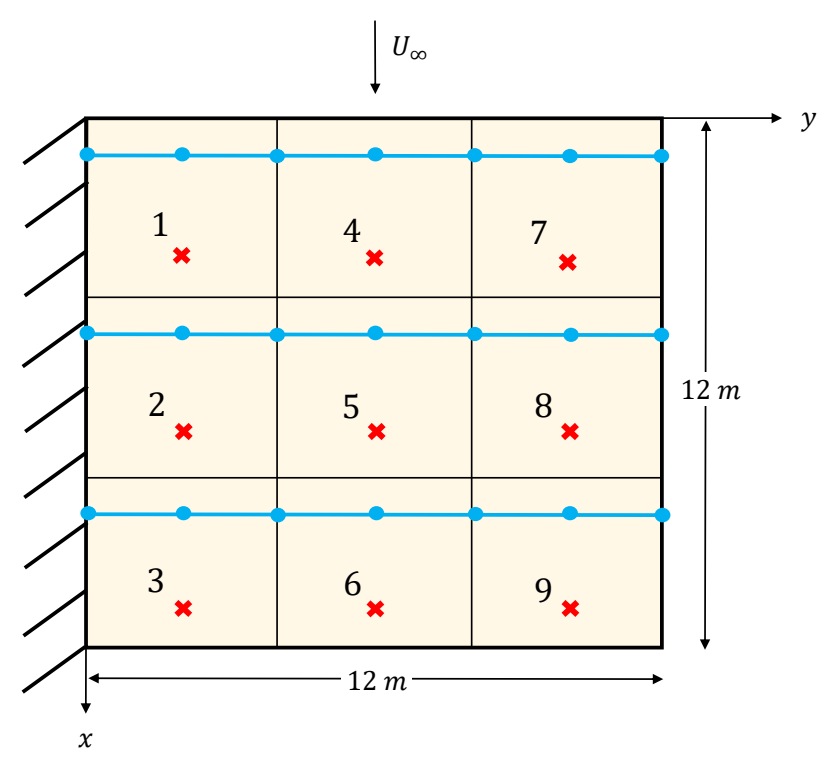

Figure 5.3: Doublet lattice method verification configuration

The wing undergoes a heave oscillation with, $w / b=1, k=1$, and $M=0.5$, where the normalwash is

$$
\{\bar{w}\}=\left\{-\frac{i k}{b} w\right\}
$$

The complex pressure coefficients for each panel is presented in Table 5.6. The pressure

Table 5.6: Complex pressure coefficients verification using DLM

\begin{tabular}{cc}
\hline Panel & $C_{p}$ \\
\hline 1 & $-0.5490+6.2682 i$ \\
2 & $-3.8862+2.4495 i$ \\
3 & $-3.8736+1.1745 i$ \\
4 & $-0.5915+5.8092 i$ \\
5 & $-3.6405+2.1530 i$ \\
6 & $-3.6234+1.0281 i$ \\
7 & $-0.5829+4.5474 i$ \\
8 & $-2.8983+1.4663 i$ \\
9 & $-2.8893+0.7119 i$ \\
\hline
\end{tabular}

coefficients agree exactly with the those produced by Blair. It should be noted that the 
form of the doublet lattice method used here integrates the steady kernel component approximately and does not add the effect of the VLM. However, because the VLM has been independently verified, it is assumed that the form of the DLM that includes the VLM steady component is acceptable.

\subsubsection{Doublet Point Method}

The doublet point method code is verified by comparison with pressure coefficient results from Laschka [26] and Ueda and Dowell [28]. In this case, numerical data is not available such that graphical curve fits are used for comparison.

The first verification involves comparing the pressure coefficients of a rectangular wing oscillating in pitching motion of unit amplitude about its midchord. The wing has an aspect ratio of $A R=2$ and oscillates at $k=1$ and $M=0$ with $\partial w / \partial x=1$, where the normalwash due to this motion is defined by

$$
\bar{w}=\left\{\frac{\partial w}{\partial x}-\frac{i k}{b}\left(b-x_{i}\right)\right\}
$$

Here, $x_{i}$ is the panel chordwise control point location. The real and imaginary components of the pressure coefficient are compared with those by Laschka [26] in Figure 5.4. The nondimensional semi-span is discretized into 5 chordwise and 10 spanwise panels.

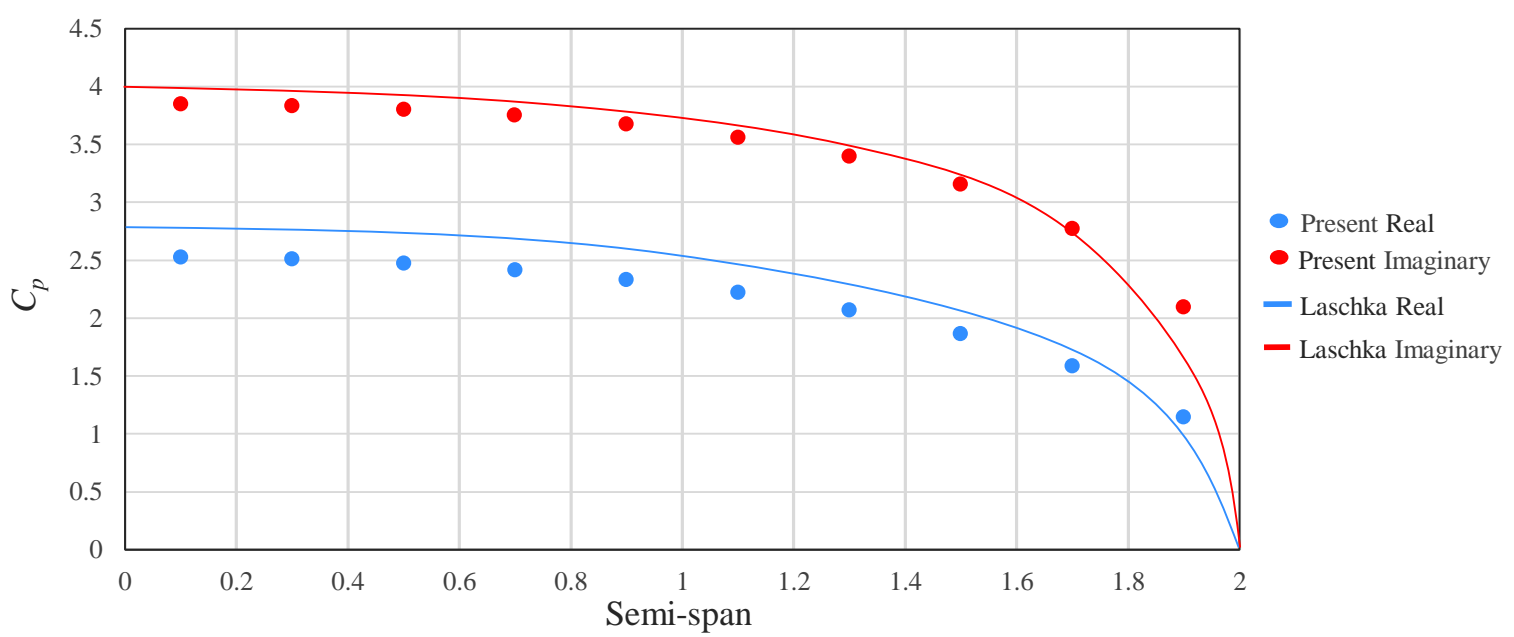

Figure 5.4: Spanwise pressure coefficient comparison using the DPM

The real and imaginary pressure coefficients generally agree, however, they are slightly different because Laschka uses the mode function method. The coefficients appear to agree exactly with the doublet point method results produced by Uedo and Dowell [28]. 
The next verification tests involves comparing the chordwise distribution of pressure coefficients along the wing root and tip for the same rectangular wing oscillating in pitching motion about the midchord. The nondimensional semi-span remains discretized into 5 chordwise and 10 spanwise panels. The real pressure coefficients for the root and tip locations are compared in Figure 5.5.

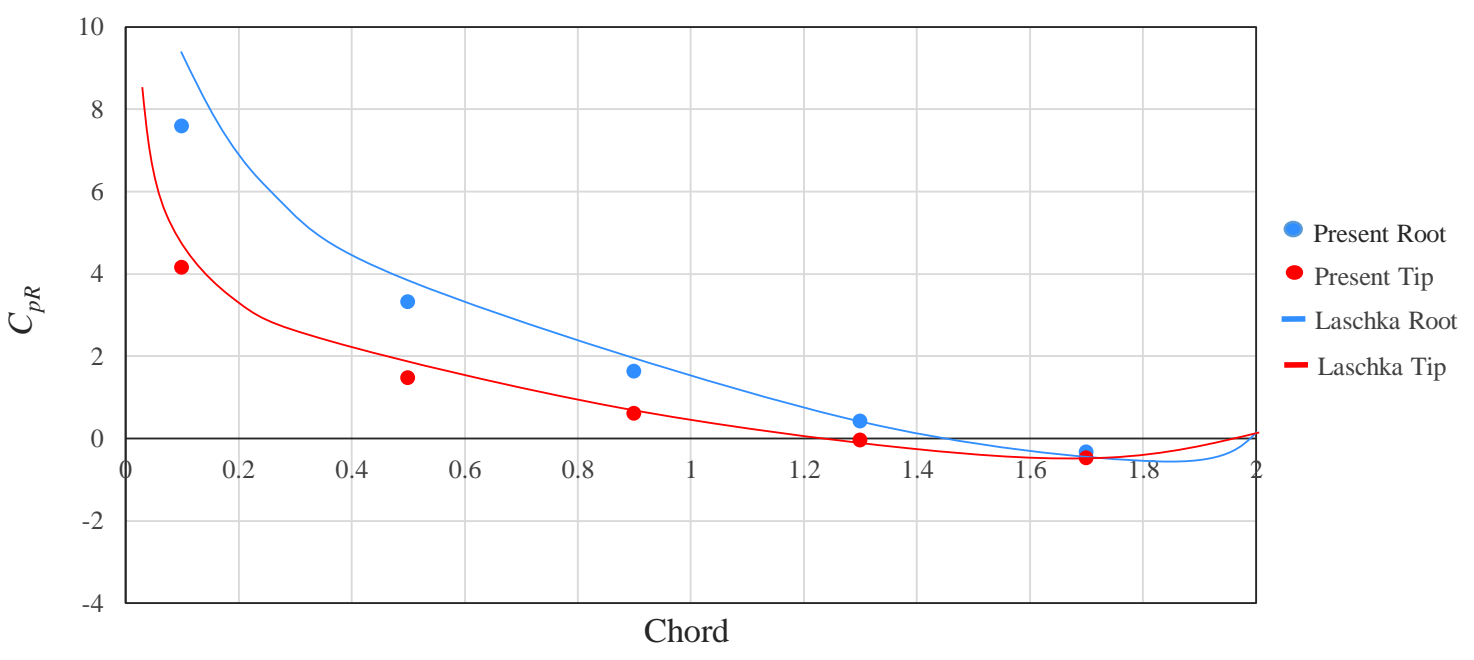

Figure 5.5: Chordwise real pressure coefficient comparison using the DPM

The imaginary pressure coefficients for the root and tip locations are compared in Figure 5.6.

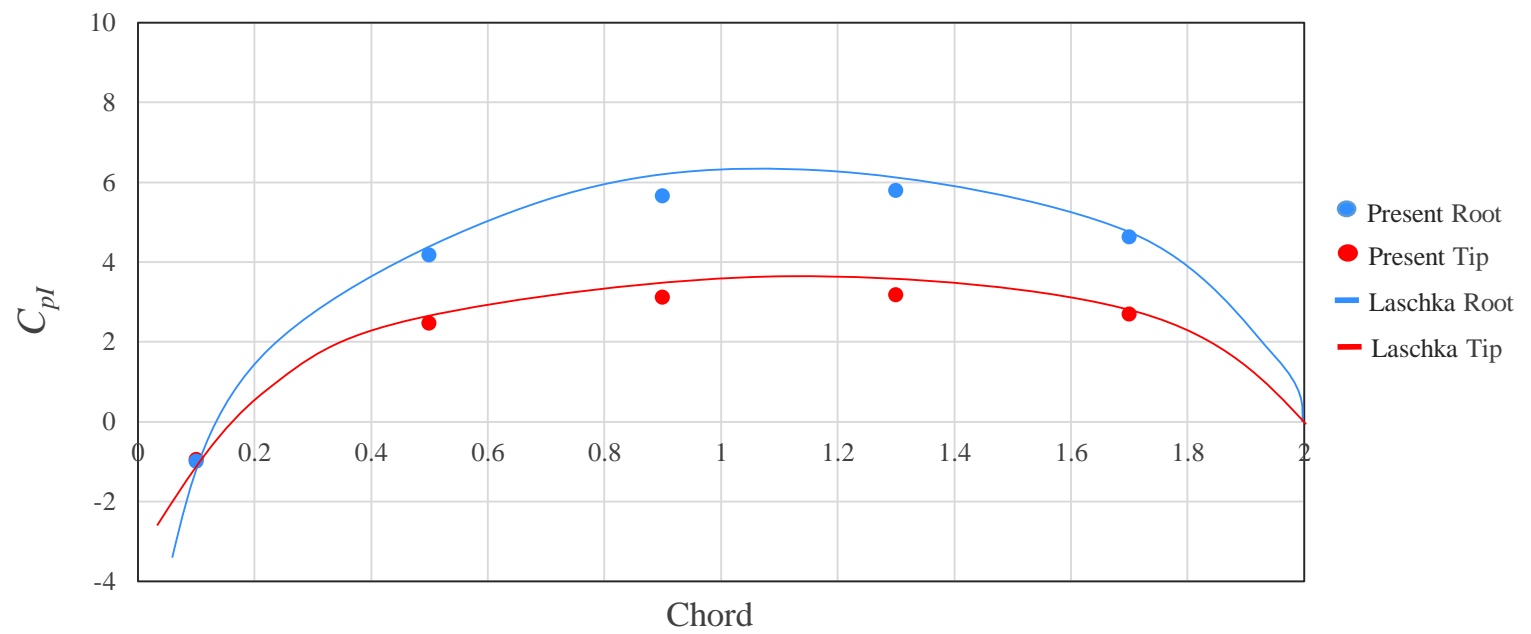

Figure 5.6: Chordwise imaginary pressure coefficient comparison using the DPM

Again, the results do not completely agree because Laschka uses the mode function method. By appearance, the chordwise pressure coefficient results agree exactly with those produced by Ueda and Dowell. Therefore, the present DPM code is considered verified. 


\subsection{Aeroelasticity Verification}

The structural and aerodynamic models have been independently verified, however, the coupling procedure and aeroelasticity solutions remain to be substantiated. The aeroelasticity verification involves comparing plate divergence and flutter speeds with available experimental and computational results.

\subsubsection{Isotropic Plate}

The aeroelasticity of an isotropic plate is first verified as a baseline. The cantilever plate geometry and coordinate system are shown in Figure 5.7.

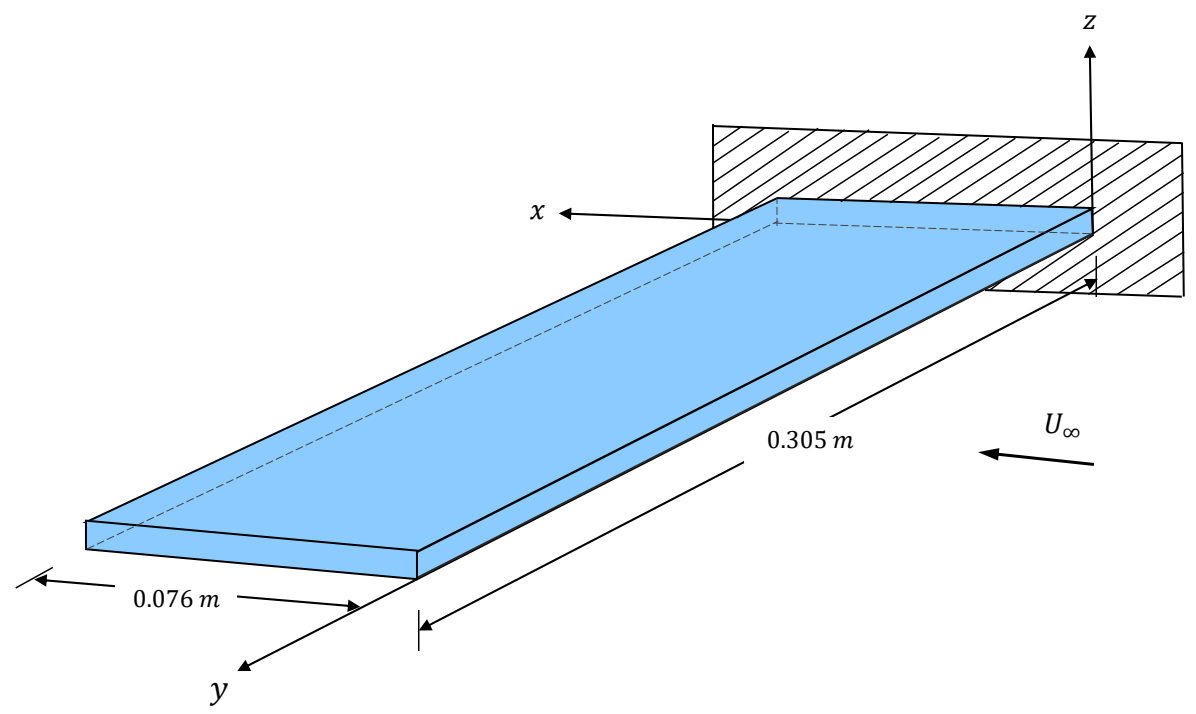

Figure 5.7: Isotropic plate geometry

The isotropic plate properties include: $E=73.8 \mathrm{GPa}, G=27.6 \mathrm{GPa}, \rho=2768 \mathrm{~kg} / \mathrm{m}^{3}$, and $t=1 \mathrm{~mm}$. The boundary conditions for the cantilevered plate at $y=0$ are:

$$
u=v=w_{b}=w_{s}=w_{b}^{x}=w_{s}^{x}=w_{b}^{y}=w_{y}^{x}=0
$$

The divergence speed of the unswept plate wing using the MONNA plate element is compared with Ref. [13] in Table 5.7.

Table 5.7: Divergence verification of isotropic plate wings

\begin{tabular}{lllc}
\hline Source & Struc. Model & Aero. Model & Divergence Speed $(\mathrm{m} / \mathrm{s})$ \\
\hline Ref. [13] & FEM $(6 \times 8)$ & VLM $(15 \times 100)$ & 84.5 \\
MONNA & FEM $(6 \times 12)$ & VLM $(6 \times 12)$ & 77.3 \\
MONNA & FEM $(6 \times 12)$ & DPM $(6 \times 12)$ & 80.4 \\
\hline
\end{tabular}


The divergence speeds generally agree with those produced by Ref. [13], which are calculated using CLPT triangular elements. The notable difference between sources could be attributed to the differences in the structural element type or the number of elements employed. The flutter speed of the isotropic plate using the MONNA element is compared with Ref. [10] and Ref. [13] in Table 5.8.

Table 5.8: Flutter verification of isotropic plate wings

\begin{tabular}{lllc}
\hline Source & Struc. Model & Aero. Model & Flutter Speed $(\mathrm{m} / \mathrm{s})$ \\
\hline Ref. [10] & FEM $(6 \times 16)$ & DPM $(6 \times 16)$ & 68 \\
Ref. [13] & FEM $(6 \times 8)$ & DLM $(8 \times 12)$ & 67.3 \\
MONNA & FEM $(6 \times 12)$ & DLM $(6 \times 12)$ & 66.6 \\
MONNA & FEM $(6 \times 12)$ & DPM $(6 \times 12)$ & 68.2 \\
\hline
\end{tabular}

The flutter speeds using the MONNA element are in good agreement with other computational results for both the VLM and DPM aerodynamic models.

\subsubsection{Composite Plates}

The aeroelasticity of composite plates are validated next. This is accomplished by comparing divergence and flutter speeds with experimental and computational results based on the specimen tested by Hollowell and Dugundji [6] with dimensions, coordinate system, and ply orientation, $\theta$, defined in Figure 5.8.

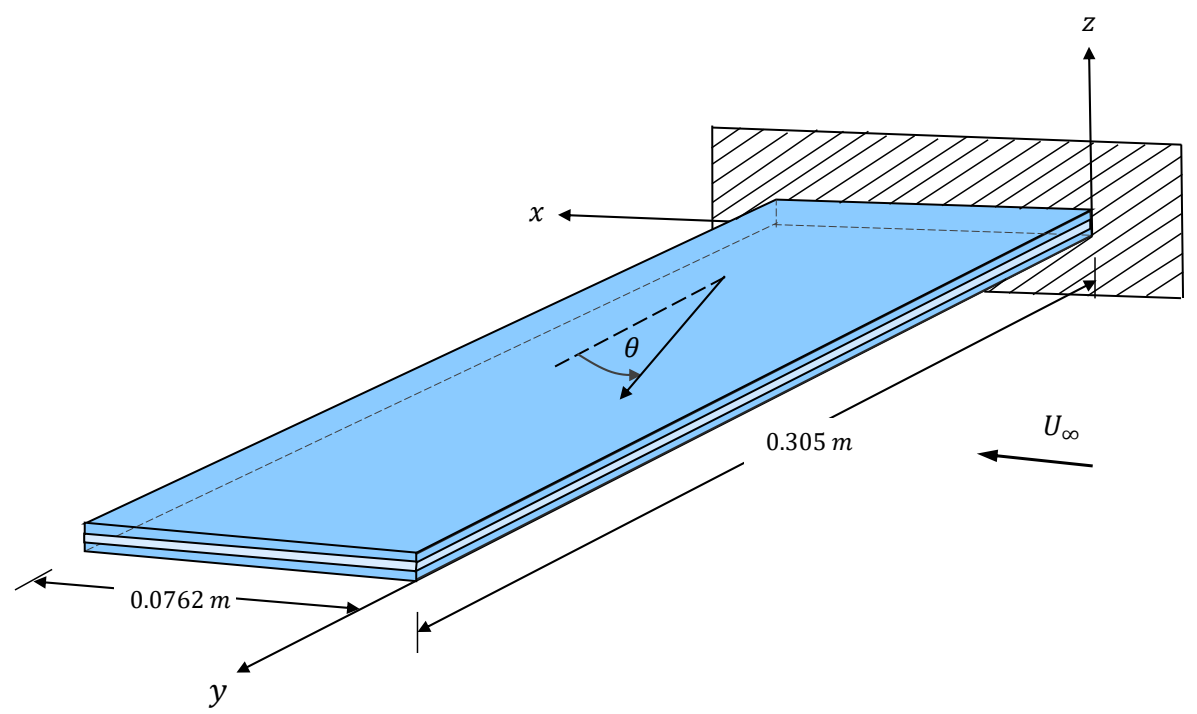

Figure 5.8: Composite verification plate geometry

The composite laminate consists of graphite/epoxy plies with the following material properties: $E_{1}=98 \mathrm{GPa}, E_{2}=7.9 \mathrm{GPa}, G_{12}=G_{13}=5.6 \mathrm{GPa}, \nu_{12}=0.28, \rho=1520$ $\mathrm{kg} / \mathrm{m}^{3}$, and $t_{p}=0.134 \mathrm{~mm}$, where $t_{p}$ is the ply thickness. The boundary conditions are 
the same as in the isotropic cantilever plate. The divergence speeds of the composite plate using the MONNA element are compared for a variety of symmetric laminates in Table 5.9.

Table 5.9: Divergence verification of composite plate wings

\begin{tabular}{lllccc}
\hline & & \multicolumn{4}{c}{ Divergence Speeds $(\mathrm{m} / \mathrm{s})$} \\
Source & Struc. Model & Aero. Model & {$\left[0_{2} / 90\right]_{s}$} & {$\left[-45_{2} / 0\right]_{s}$} & {$\left[-30_{2} / 0\right]_{s}$} \\
\hline Ref. [6] & Exp. & Exp. & - & 12.5 & 11.7 \\
Ref. [13] & FEM $(6 \times 8)$ & VLM $(15 \times 100)$ & 25.4 & 12.7 & 12.8 \\
Ref. [11] & FEM $(4 \times 16)$ & DPM $(6 \times 8)$ & 30.6 & 13.7 & 13.8 \\
MONNA & FEM $(6 \times 8)$ & VLM $(6 \times 8)$ & 27.8 & 12.9 & 13.1 \\
MONNA & FEM $(6 \times 8)$ & DPM $(6 \times 8)$ & 30.6 & 13.6 & 13.8 \\
\hline
\end{tabular}

The MONNA element model divergence speeds are in good agreement with the experimental and computational results with both the VLM and DPM aerodynamic models. The results indicate that the DPM tends to over predict the experimental divergence speeds relative to the VLM or DLM. The flutter speeds of the composite plate are compared for a variety of symmetric laminates in Table 5.10.

Table 5.10: Flutter verification of composite plate wings

\begin{tabular}{lllcccc}
\hline & & & \multicolumn{3}{c}{ Flutter Speeds $(\mathrm{m} / \mathrm{s})$} \\
Source & Struc. Model & Aero. Model & {$\left[0_{2} / 90\right]_{s}$} & {$\left[45_{2} / 0\right]_{s}$} & {$\left[30_{2} / 0\right]_{s}$} & {$[45 /-45 / 0]_{\mathrm{s}}$} \\
\hline Ref. [6] & Exp. & Exp. & 25 & 28 & 27 & $>32$ \\
Ref. [13] & FEM $(6 \times 8)$ & DLM $(8 \times 12)$ & 26.4 & 27.8 & 27.4 & 47.5 \\
Ref. [7] & FEM $(3 \times 8)$ & DLM $(6 \times 8)$ & 21.8 & - & 24.9 & - \\
Ref. [8] & FEM $(2 \times 6)$ & DPM $(6 \times 8)$ & 22.9 & 27.6 & 27.2 & - \\
MONNA & FEM $(6 \times 8)$ & DLM $(6 \times 8)$ & 21.9 & 25.8 & 25.4 & 38.1 \\
MONNA & FEM $(6 \times 8)$ & DPM $(6 \times 8)$ & 22.9 & 27.5 & 27.1 & 39.8 \\
\hline
\end{tabular}

The MONNA flutter speeds are in good agreement with experimental and computational results, although the DLM tends to under predict the experimental flutter speeds. The present DPM results are similar to the DPM results by Ref. [8]. The present DLM results are similar to Ref. [7], however, they are noticeably different from Ref. [13], who consistently produced higher flutter speeds relative to any other source that employed the DLM.

An attempt may be made to account for this discrepancy by considering that Ref. [13] is using CLPT triangular elements rather than HSDT rectangular elements, however, Ref. [7] is also using CLPT triangular elements. The argument may be made that Ref.[13] is using a higher number of aerodynamic elements, but increasing the 
aerodynamic mesh size to $8 \times 12$ in the present model changes the solution minimally. It is proposed the discrepancy is primarily attributed to the way in which Ref. [13] performs the aeroelastic load transformation. Ref. [13] indicates the doublet lattice loads are being treated as pressures acting uniformly across the panels rather than line loads across the panel quarter chord, which is not consistent with the doublet lattice method [25], [31], [32]. This could account for the relatively high DLM flutter speeds produced by Ref. [13] because treating the doublet lattice loads as uniform pressures decreases the overall moment experienced by the wing and consequentially increases flutter speed.

In order to demonstrate the flutter solution methodology, the $V-g$ and $V-\omega$ plots for the DPM solution with the $\left[0_{2} / 90\right]$ laminate are shown, for example, in Figures 5.9 and 5.10 , respectively. The flutter speed of $22.9 \mathrm{~m} / \mathrm{s}$ is given by referencing the lowest speed at which one of the modes damping coefficient becomes zero in Figure 5.9. The equivalent location in Figure 5.10 is the associated frequency at which the flutter occurs. As previously stated, the $k$-method solution does not provide as accurate damping trends as the $p$ - $k$-method, but they both provide the same estimate speed at the flutter condition. [2].

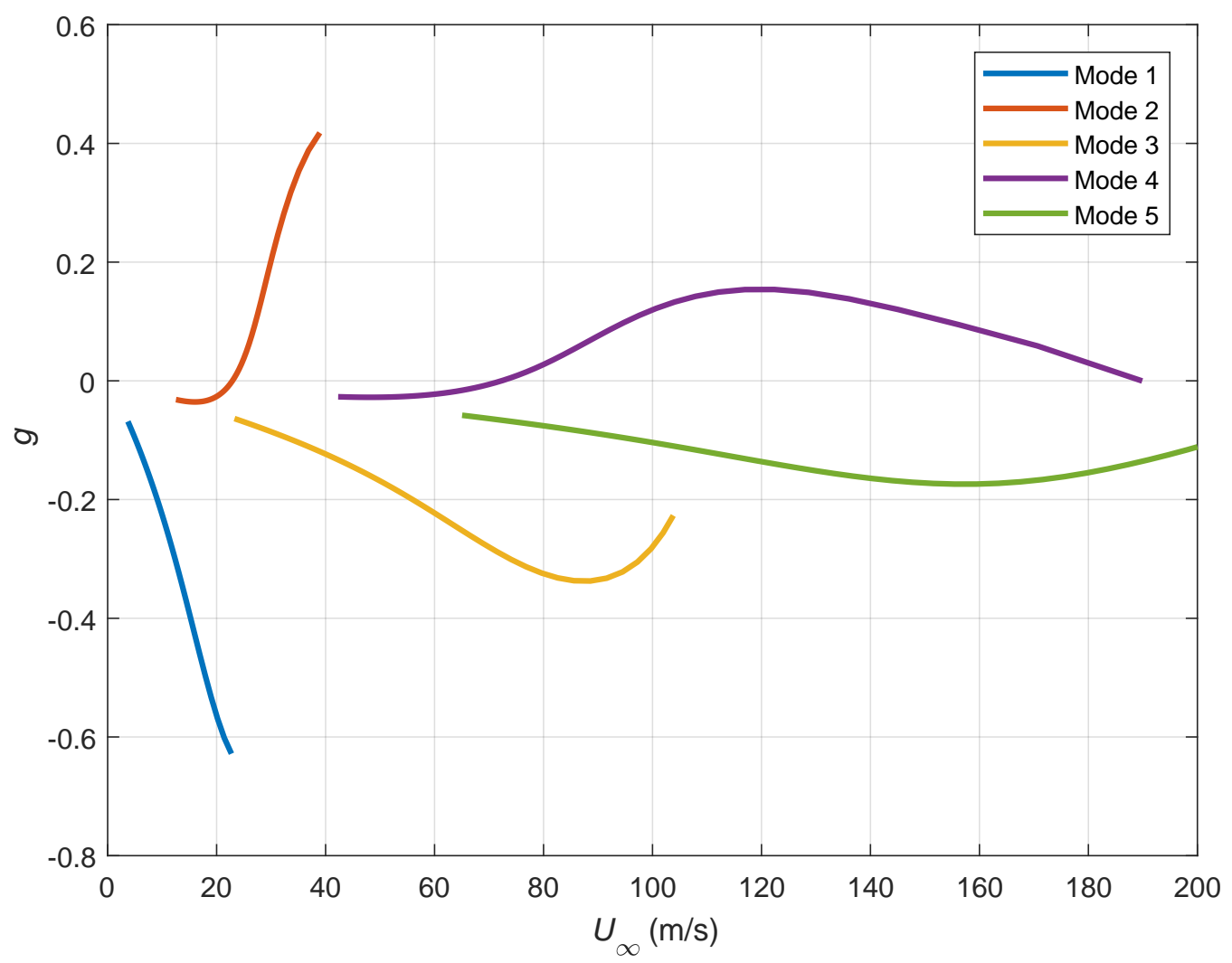

Figure 5.9: $V-g$ plot for $\left[0_{2} / 90\right]$ laminate using the DPM

The composite plate divergence and flutter speeds of the MONNA plate element using the DLM have been first presented by the present author in Ref. [33]. The 


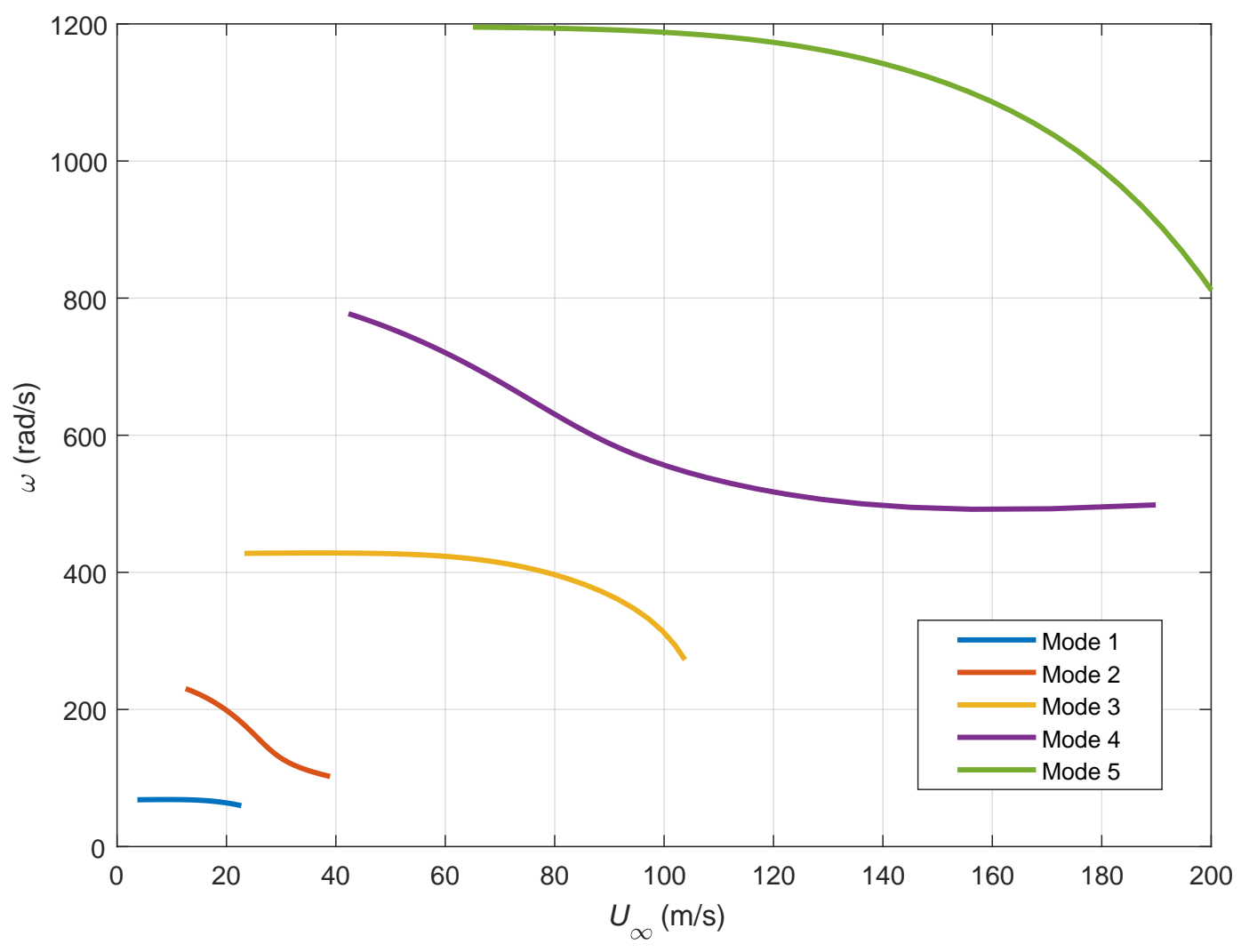

Figure 5.10: $V-\omega$ plot for $\left[0_{2} / 90\right]$ laminate using the DPM

verification results from Table 5.10 indicate that the DPM produced more accurate flutter speeds compared to the DLM with the present model. For this reason, the DPM is primarily employed as the aerodynamic model for the flutter research in the remainder of this thesis. 


\section{Chapter 6}

\section{Results and Discussion}

In this chapter, the aeroelastic performance of the MONNA plate element is analyzed. A convergence study of the MONNA element is first performed, followed by a comparison in aeroelastic results between HSDT and FSDT and a parametric study.

\subsection{Aeroelastic Convergence Study}

An investigation is performed on the flutter convergence characteristics of the new conforming $h-p$ version MONNA element relative to the conforming BFS and nonconforming Melosh elements using the aeroelastic codes that have been developed for all three elements. For this test, the same composite plate and material properties are used as in the composite aeroelasticity validation of Section 5.3.2. This composite plate is based off of the experimental specimen of Hollowell and Dugundji [6].

The first convergence study is performed on a symmetric cross-ply laminate and is shown in Table 6.1, where the structural elements vary and the aerodynamic elements are kept constant at $8 \times 12$.

Table 6.1: Convergence study for $\left[0_{2} / 90\right]_{s}$ laminate $(8 \times 12$ DPM elements $)$

\begin{tabular}{cccc}
\hline & \multicolumn{3}{c}{ Flutter Speeds $(\mathrm{m} / \mathrm{s})$} \\
Struc. Elements & MONNA Element & BFS Element & Melosh Element \\
\hline $1 \times 2$ & 23.18 & 23.34 & 23.34 \\
$2 \times 4$ & 23.16 & 23.18 & 23.18 \\
$4 \times 6$ & 23.16 & 23.16 & 23.17 \\
$8 \times 12$ & 23.16 & 23.16 & 23.16 \\
\hline
\end{tabular}

The MONNA plate element demonstrated the best convergence performance, as the flutter speed converged to four significant digits with $2 \times 4$ elements and was within $0.086 \%$ of the converged solution with only $1 \times 2$ elements. The conforming BFS element and nonconforming Melosh element required $4 \times 6$ and $8 \times 12$ elements to 
converge, respectively. This comparison is shown in Figure 6.1, where the convergence behavior of the BFS and Melosh elements are very similar.

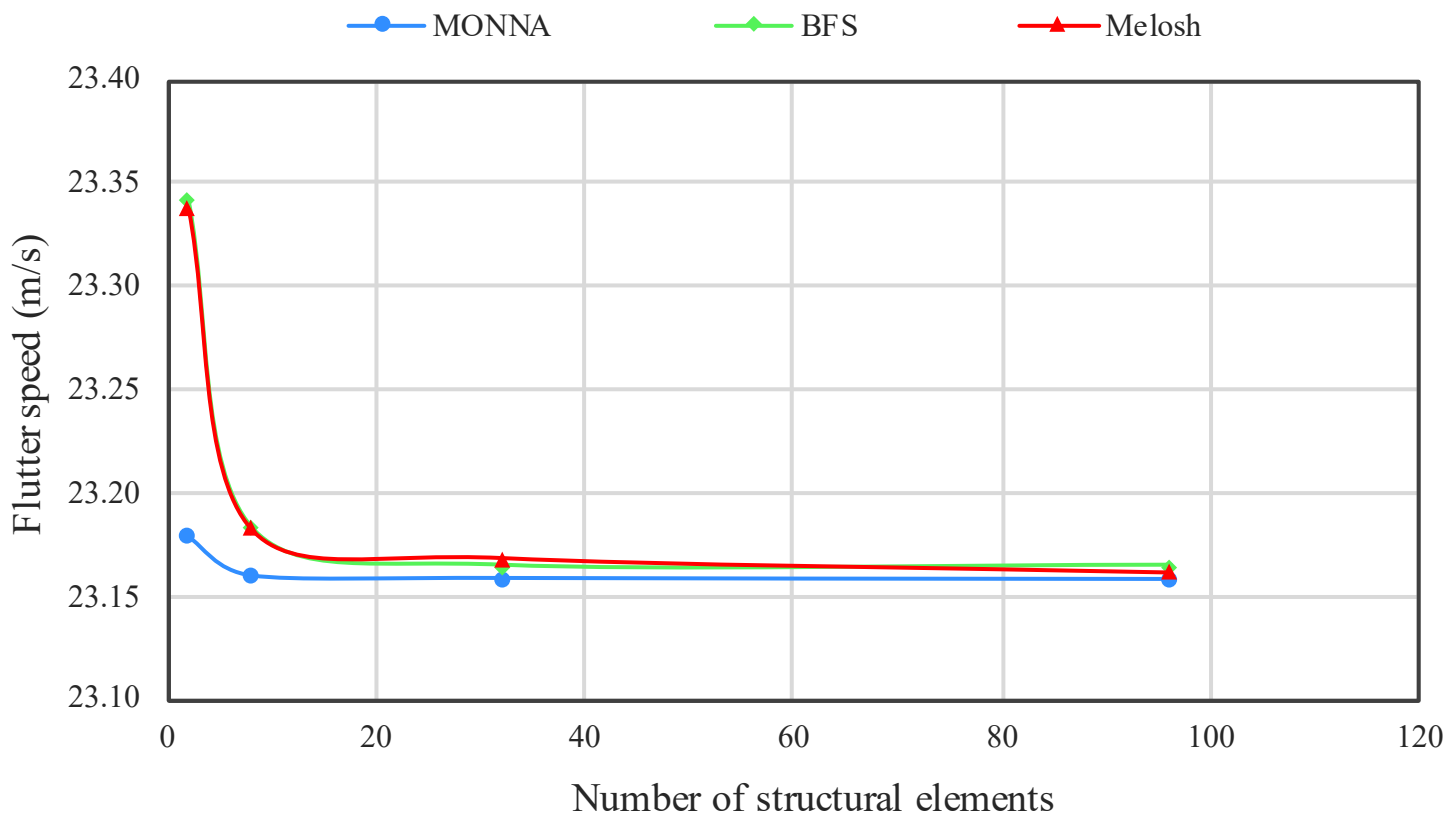

Figure 6.1: Convergence study of $\left[0_{2} / 90\right]_{s}$ laminate

The convergence study of an unsymmetric cross-ply laminate is shown in Table 6.2. Similar to the symmetric laminate, the MONNA element demonstrated the best Table 6.2: Convergence study for $[0 / 90]_{3}$ laminate $(8 \times 12$ DPM elements $)$

\begin{tabular}{cccc}
\hline & \multicolumn{3}{c}{ Flutter Speeds $(\mathrm{m} / \mathrm{s})$} \\
Struc. Elements & MONNA Element & BFS Element & Melosh Element \\
\hline $1 \times 2$ & 22.69 & 22.95 & 22.96 \\
$2 \times 4$ & 22.66 & 22.72 & 22.72 \\
$4 \times 6$ & 22.65 & 22.68 & 22.68 \\
$8 \times 12$ & 22.65 & 22.66 & 22.66 \\
\hline
\end{tabular}

convergence. The the performance of the BFS and Melosh elements are again, nearly identical. The overall performance of each element changed little between the symmetric and unsymmetric cross-ply laminates.

The convergence study for a symmetric laminate with $45^{\circ}$ plies are shown in Table 6.3. In this case, the Melosh element is surprisingly accurate even at a low number of structural elements. The BFS element performance is the worst among the three, however, its solution became similar to the others by $8 \times 12$ elements. This behavior is shown in Figure 6.2, where the data of Table 6.3 is plotted. Each element does not appear to be fully converged even at $8 \times 12$ elements. This could indicate the unbalancing of the laminate is responsible for delaying convergence relative to the 
Table 6.3: Convergence study for $\left[45_{2} / 0\right]_{s}$ laminate $(8 \times 12$ DPM elements $)$

$$
\text { Flutter Speeds }(\mathrm{m} / \mathrm{s})
$$

\begin{tabular}{cccc} 
Struc. Elements & MONNA Element & BFS Element & Melosh Element \\
\hline $1 \times 2$ & 27.78 & 28.65 & 27.51 \\
$2 \times 4$ & 27.37 & 27.70 & 27.33 \\
$4 \times 6$ & 27.24 & 27.44 & 27.30 \\
$8 \times 12$ & 27.15 & 27.22 & 27.18 \\
\hline
\end{tabular}

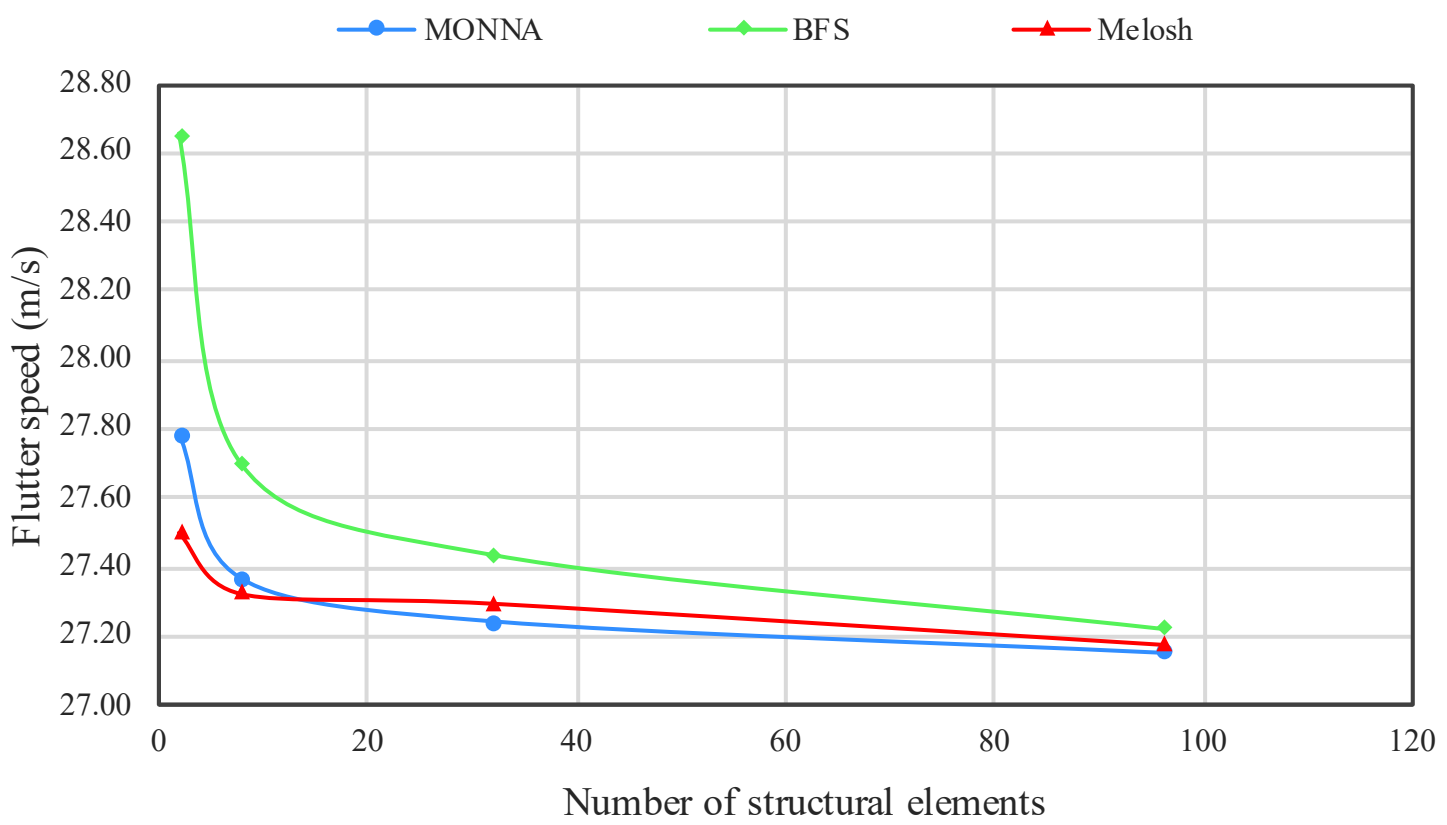

Figure 6.2: Convergence study of $\left[45_{2} / 0\right]_{s}$ laminate

balanced cross-ply laminate. Although the Melosh element is the most accurate at low number of elements, the MONNA element seems to ultimately approach the converged solution the fastest.

The convergence study of an unsymmetric laminate with $45^{\circ}$ plies is shown in Table 6.4. For this laminate, MONNA element demonstrated the best performance,

Table 6.4: Convergence study for $[0 / 45]_{3}$ laminate $(8 \times 12$ DPM elements $)$

$$
\text { Flutter Speeds }(\mathrm{m} / \mathrm{s})
$$

\begin{tabular}{cccc} 
Struc. Elements & MONNA Element & BFS Element & Melosh Element \\
\hline $1 \times 2$ & 28.18 & 28.87 & 28.65 \\
$2 \times 4$ & 27.95 & 28.16 & 28.09 \\
$4 \times 6$ & 27.92 & 28.01 & 28.01 \\
$8 \times 12$ & 27.90 & 27.92 & 27.93 \\
\hline
\end{tabular}

followed by the Melosh element. The BFS element provided the most inaccurate result 
when $1 \times 2$ elements were employed, but it quickly matched results produced by the Melosh element as the number of elements increased. The data of Table 6.4 is plotted in Figure 6.3 to illustrate this behavior. The flutter speeds from Table 6.4 indicate that

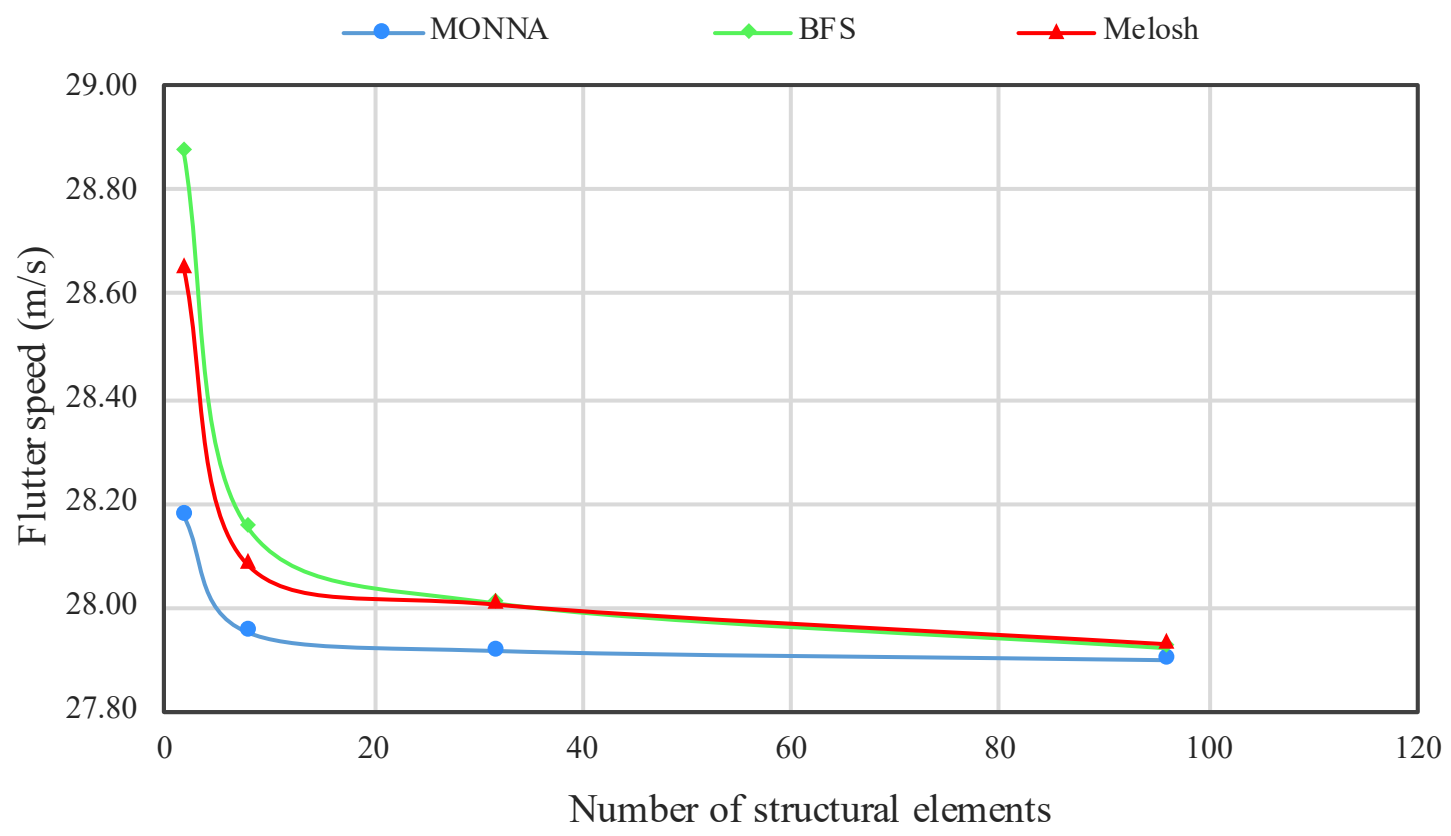

Figure 6.3: Convergence study of $[0 / 45]_{3}$ laminate

none of the elements had fully converged to four significant digits by the application of $8 \times 12$ elements. Again, this is probably due to the laminate being unbalanced.

The convergence study of a symmetric laminate with both $45^{\circ}$ and $-45^{\circ}$ plies is shown in Table 6.5 The behavior of each element, as plotted in Figure 6.4, is similar to

Table 6.5: Convergence study for $[45 /-45 / 0]_{\mathrm{s}}$ laminate $(8 \times 12$ DPM elements $)$

\begin{tabular}{cccc}
\hline & \multicolumn{3}{c}{ Flutter Speeds $(\mathrm{m} / \mathrm{s})$} \\
Struc. Elements & MONNA Element & BFS Element & Melosh Element \\
\hline $1 \times 2$ & 29.63 & 30.04 & 29.94 \\
$2 \times 4$ & 29.56 & 29.63 & 29.60 \\
$4 \times 6$ & 29.55 & 29.57 & 29.57 \\
$8 \times 12$ & 29.55 & 29.55 & 29.55 \\
\hline
\end{tabular}

that of the unsymmetric $[0 / 45]_{3}$ laminate. Curiously, the MONNA plate element solution appears to be fully converged by $4 \times 6$ elements in a similar manner to the balanced cross-ply laminates. This observation provides additional evidence that the flutter convergence is faster for balanced laminates.

Overall, the convergence behavior of each element is undoubtedly dependent on the laminate symmetry and ply orientation. The MONNA element demonstrated fast and superior convergence when balanced laminates are analyzed, while the low element 


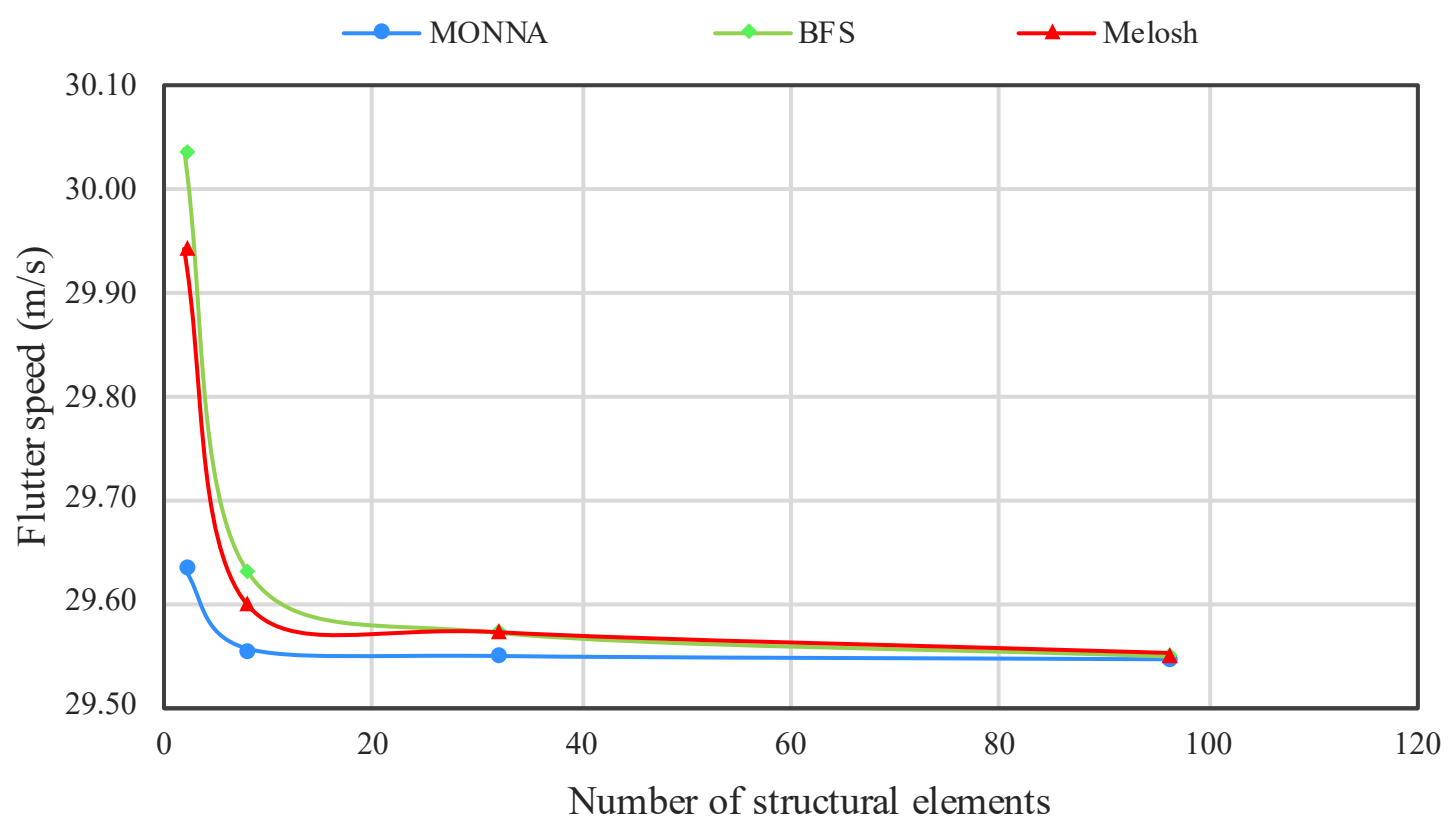

Figure 6.4: Convergence study of $[45 /-45 / 0]_{\mathrm{s}}$ laminate

performance of the nonconforming Melosh element is more accurate than the conforming BFS element for laminates with angle plies. The nonconforming Melosh element is surprisingly accurate in the context of aeroelasticity considering its poor performance in the structural validation of Section 5.1.2. Nonetheless, it may be concluded the MONNA element provided the most rapid and consistent convergence across all laminates. It also concluded that at least $4 \times 6$ structural elements should be used for a converged solution while using the MONNA element in an aeroelastic analysis. 


\subsection{Effect of HSDT and Laminate Thickness}

The effect of HSDT and laminate thickness on the flutter of composite plates is determined by calculating flutter speeds for both FSDT and HSDT while varying laminate thickness. In the present study, three different materials with properties representative of graphiteepoxy composites are used for this purpose. The properties for these materials are chosen based on moduli ratios and presented in Table 6.6.

Table 6.6: Composite material properties for laminate thickness and HSDT analysis

\begin{tabular}{cccc}
\hline Property & Material 1 & Material 2 & Material 3 \\
\hline$E_{1}(\mathrm{GPa})$ & 130 & 150 & 160 \\
$E_{2}(\mathrm{GPa})$ & 10.4 & 6.0 & 4.0 \\
$G_{12}(\mathrm{GPa})$ & 5.2 & 3.0 & 2.4 \\
$G_{13}(\mathrm{GPa})$ & 5.2 & 3.0 & 2.4 \\
$G_{23}(\mathrm{GPa})$ & 3.1 & 1.8 & 1.4 \\
$\nu_{12}$ & 0.28 & 0.28 & 0.28 \\
$\rho\left(\mathrm{kg} / \mathrm{m}^{3}\right)$ & 1600 & 1600 & 1600 \\
\hline
\end{tabular}

Material 1 is representative of a common graphite-epoxy, such as T300-914 [34], where the following moduli ratios are assumed: $E_{1}=12.5 E_{2}$ and $G_{12}=0.5 E_{2}$. The properties of Material 2 are based on ratios employed by Reddy [18] for the analysis of HSDT composite plate deflection: $E_{1}=25 E_{2}$ and $G_{12}=0.5 E_{2}$. Materials 3 is based on ratios used by Reddy for HSDT composite plate natural frequency analysis: $E_{1}=40 E_{2}$ and $G_{12}=0.6 E_{2}$. These different materials are included to provide results for composites materials with varying shear moduli.

The geometry of the composite specimen used for this analysis is shown in Figure 6.5. This composite plate is similar to that used by Hollowell and Dugundji [6], except

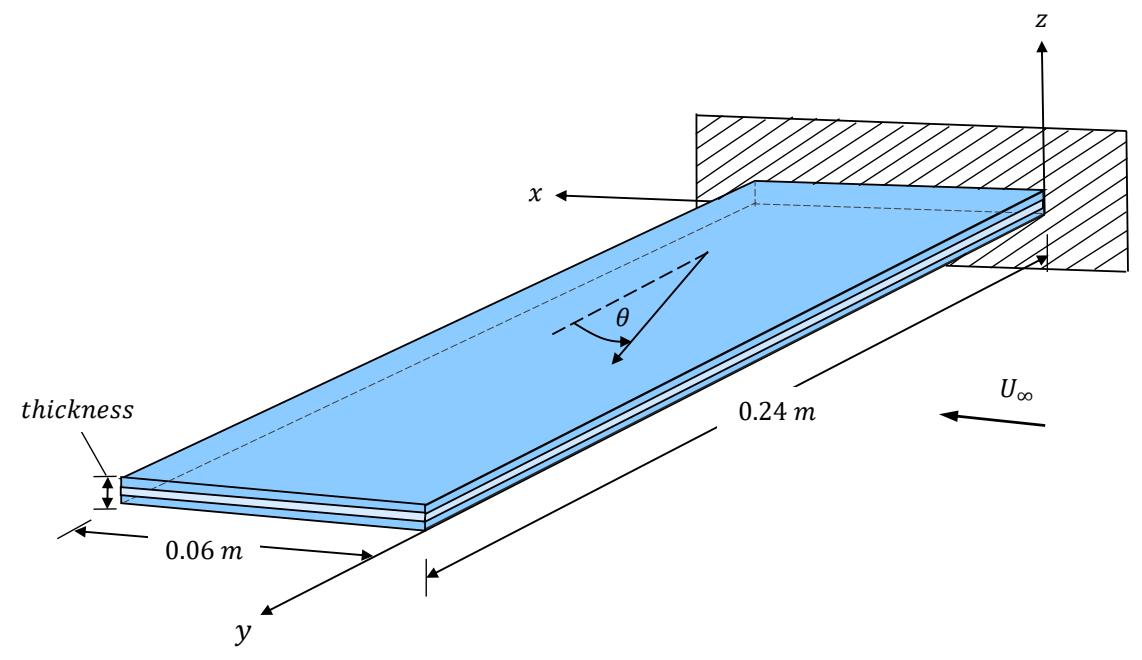

Figure 6.5: Composite plate geometry for laminate thickness and HSDT analysis 
the dimensions are changed to achieve specific chord to thickness ratios. The effect of laminate thickness is studied for both divergence and flutter speeds by keeping the plate length and width constant and steadily increasing laminate thickness, where the ply thickness is $t_{p}=1 \times 10^{-4} \mathrm{~m}$. The shear correction factor is set to $K_{s}=4 / 5$ and $K_{s}=1$ for FSDT and HSDT, respectively.

\section{Divergence Analysis}

The plate divergence speeds are calculated using the VLM aerodynamic model. In every case, the analysis is performed with $6 \times 8$ aerodynamic elements and $6 \times 8$ structural elements. Table 6.7 records symmetric cross-ply laminate divergence speeds for all three materials and for chord-to-thickness ratios of $c / t=100,50,25$ with both FSDT and HSDT. The data in Table 6.7 is plotted in Figure 6.6.

Table 6.7: Divergence speed analysis with $\left[0_{2 n} / 90_{n}\right]_{s}$ laminate

\begin{tabular}{cccccc}
\hline & \multicolumn{5}{c}{ Divergence Speeds $(\mathrm{m} / \mathrm{s})$} \\
Material & $n$ & $c / t$ & HSDT & FSDT & $\%$ Diff. \\
\hline 1 & 1 & 100 & 25.771 & 25.771 & $0.0001 \%$ \\
& 2 & 50 & 72.889 & 72.889 & $0.0004 \%$ \\
& 4 & 25 & 206.143 & 206.140 & $0.0015 \%$ \\
\hline 2 & 1 & 100 & 21.503 & 21.503 & $0.0005 \%$ \\
& 2 & 50 & 60.817 & 60.817 & $0.0003 \%$ \\
& 4 & 25 & 171.997 & 171.994 & $0.0016 \%$ \\
\hline 3 & 1 & 100 & 18.973 & 18.973 & $0.0011 \%$ \\
& 2 & 50 & 53.662 & 53.662 & $0.0006 \%$ \\
& 4 & 25 & 151.759 & 151.757 & $0.0016 \%$ \\
\hline
\end{tabular}

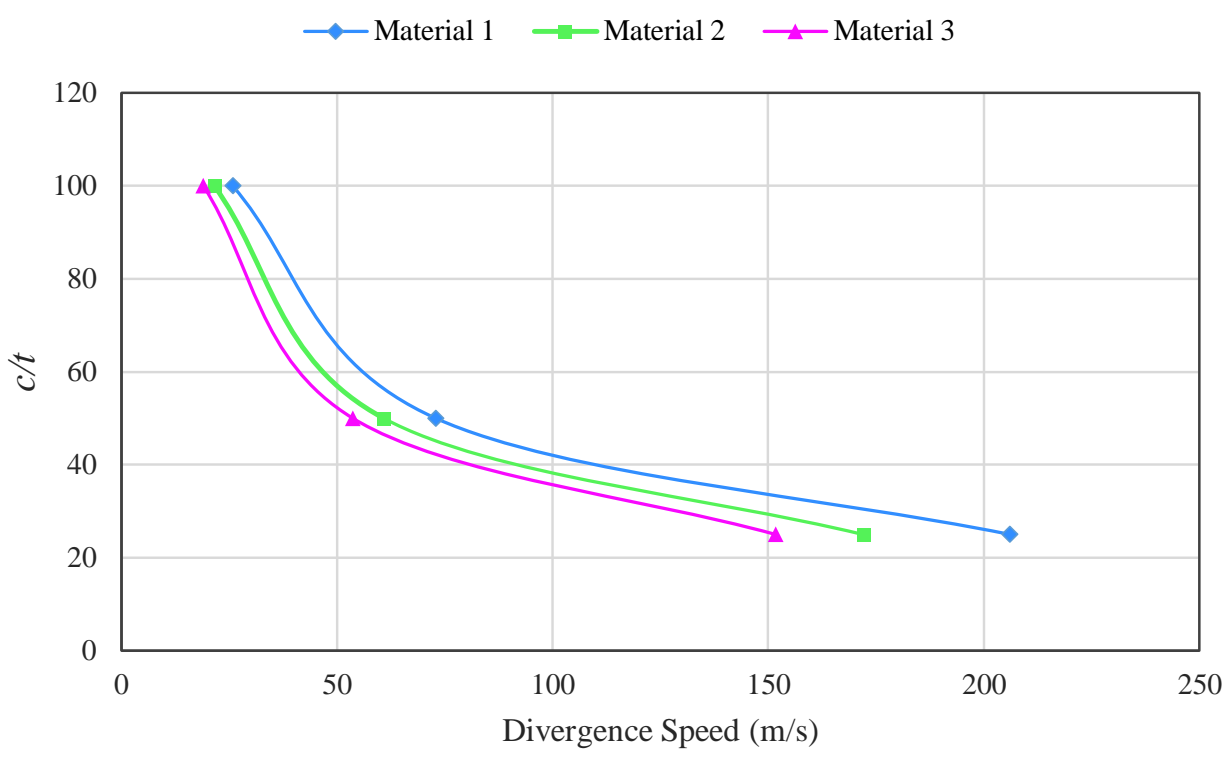

Figure 6.6: HSDT divergence speed analysis with $\left[0_{2 n} / 90_{n}\right]_{s}$ laminate 
This analysis reveals extremely small difference in divergence speeds between the HSDT and FSDT formulations even with $c / t=25$. The largest percent difference between the two theories is merely $0.0016 \%$, where the application of HSDT slightly increased divergence speed. As anticipated, divergence speeds increased significantly with decrease in chord-to-thickness ratio because plate torsional rigidity is greatly increased with increased thickness. Material 1 experienced the highest divergence speeds, while Material 3 experienced the lowest speeds across all chord-to-thickness ratios. This occurs because torsional rigidity is also a function of shear moduli, which is highest in Material 1 and lowest in Material 3.

The same divergence analysis is performed on a symmetric laminate with $-45^{\circ}$ plies in Table 6.8. The data in Table 6.8 is plotted in Figure 6.7.

Table 6.8: Divergence speed analysis with $\left[-45_{2 n} / 0_{n}\right]_{s}$ laminate

\begin{tabular}{cccccc}
\hline \multirow{5}{*}{ Material } & $n$ & $c / t$ & HSDT & FSDT & $\%$ Diff. \\
\hline 1 & 1 & 100 & 13.500 & 13.500 & $0.0000 \%$ \\
& 2 & 50 & 38.183 & 38.183 & $0.0002 \%$ \\
& 4 & 25 & 107.994 & 107.993 & $0.0009 \%$ \\
\hline 2 & 1 & 100 & 11.135 & 11.135 & $0.0001 \%$ \\
& 2 & 50 & 31.493 & 31.493 & $0.0000 \%$ \\
& 4 & 25 & 89.072 & 89.071 & $0.0009 \%$ \\
\hline 3 & 1 & 100 & 9.905 & 9.905 & $0.0000 \%$ \\
& 2 & 50 & 28.015 & 28.015 & $0.0003 \%$ \\
& 4 & 25 & 79.236 & 79.235 & $0.0009 \%$ \\
\hline
\end{tabular}

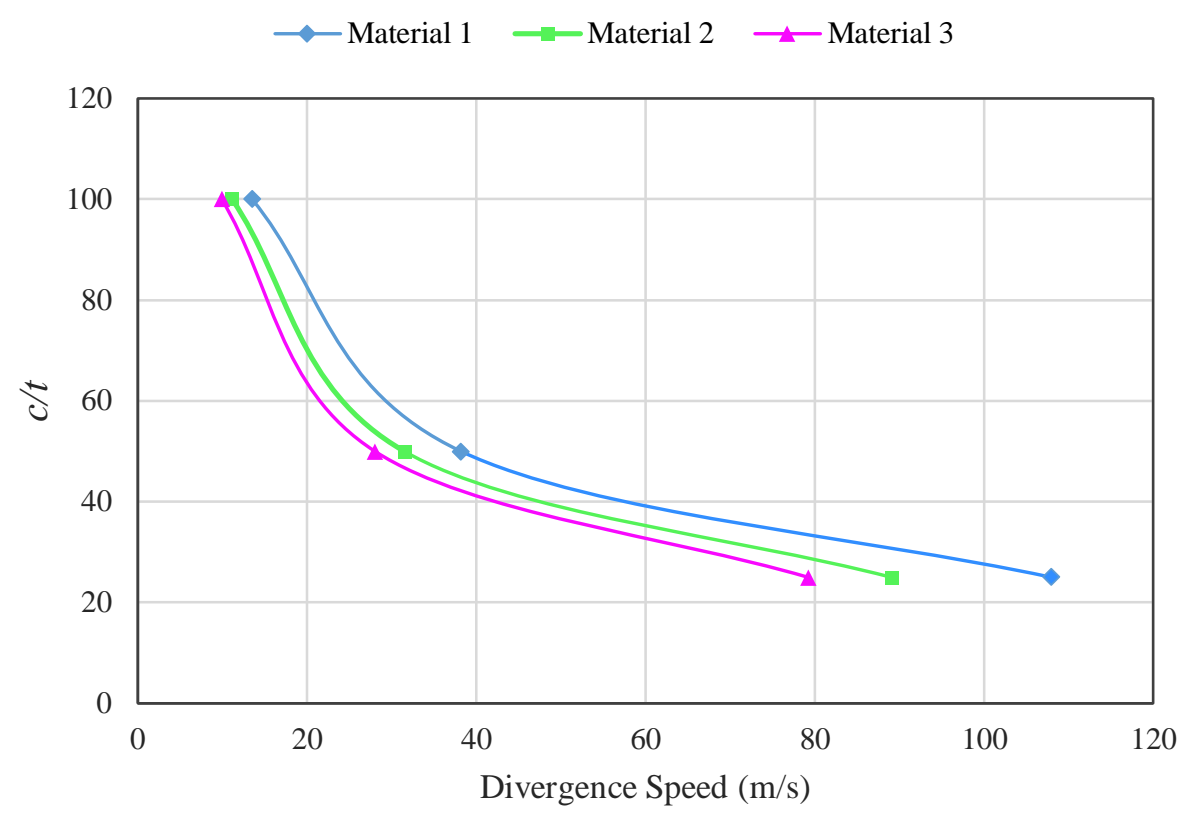

Figure 6.7: HSDT divergence speed analysis with $\left[-45_{2 n} / 0_{n}\right]_{s}$ laminate 
The same trends are seen in the $\left[-45_{2 n} / 0_{n}\right]_{s}$ laminate as in the $\left[0_{2 n} / 90_{n}\right]_{s}$ laminate. The percent difference between HSDT and FSDT speeds are negligible and the divergence speeds are highest for Material 1 and lowest for Material 3 for the same reasons as before. The divergence speeds are significantly less for the angled laminate compared to the cross-ply laminate. The reason for this behavior is explored in the following section.

\section{Flutter Analysis}

The plate wing flutter analysis is performed using the DPM aerodynamic model. In this study, $4 \times 6$ structural elements and $8 \times 12$ aerodynamic elements are used. Table 6.9 records symmetric cross-ply laminate flutter speeds for all three materials and for chord-to-thickness ratios of $c / t=100,50,25$ with both FSDT and HSDT. The data in Table 6.9 is plotted in Figure 6.8.

Table 6.9: Flutter speed analysis with $\left[0_{2 n} / 90_{n}\right]_{s}$ laminate

\begin{tabular}{cccccc}
\hline & \multicolumn{5}{c}{ Flutter Speeds $(\mathrm{m} / \mathrm{s})$} \\
Material & $n$ & $c / t$ & HSDT & FSDT & $\%$ Diff. \\
\hline 1 & 1 & 100 & 21.031 & 21.031 & $0.0001 \%$ \\
& 2 & 50 & 59.367 & 59.366 & $0.0005 \%$ \\
& 4 & 25 & 170.208 & 170.204 & $0.0019 \%$ \\
\hline 2 & 1 & 100 & 16.677 & 16.677 & $0.0002 \%$ \\
& 2 & 50 & 46.922 & 46.922 & $0.0009 \%$ \\
& 4 & 25 & 134.716 & 134.712 & $0.0036 \%$ \\
\hline 3 & 1 & 100 & 14.052 & 14.052 & $0.0004 \%$ \\
& 2 & 50 & 39.340 & 39.340 & $0.0018 \%$ \\
& 4 & 25 & 112.710 & 112.701 & $0.0077 \%$ \\
\hline
\end{tabular}

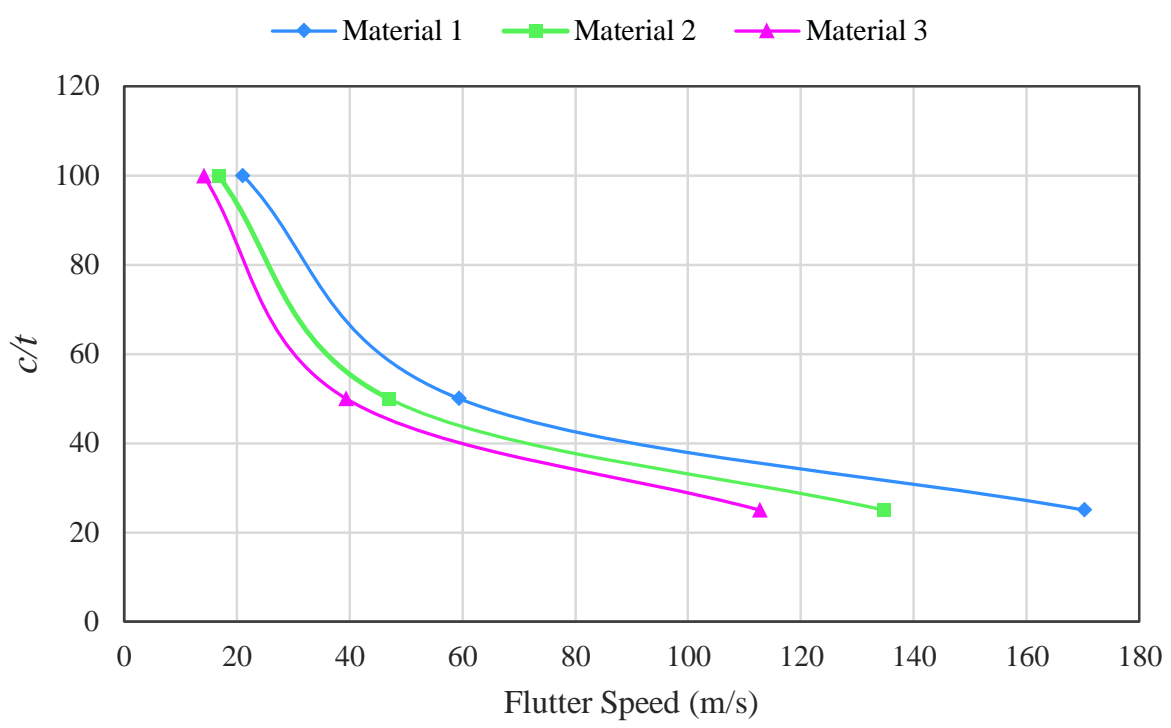

Figure 6.8: HSDT flutter speed analysis with $\left[0_{2 n} / 90_{n}\right]_{s}$ laminate 
The flutter analysis demonstrates negligible difference between HSDT and FSDT formulations even for thick laminates with $c / t=25$ and materials with extreme moduli ratios. The highest percent difference between the formulations occurs in Material 3 at only $0.0077 \%$, where the application of HSDT slightly increased flutter speed. The differences between the formulations steadily increased from Material 1 to Material 3. The flutter speeds increase significantly with thickness, which is due to an increased bending stiffness and torsional rigidity. Similar to what is found in the divergence analysis, lower material shear moduli attributed to lower torsional rigidity and consequentially, lower flutter speeds.

The same flutter analysis is performed on a symmetric laminate comprising of $45^{\circ}$ plies in Table 6.10. The data in Table 6.10 is plotted in Figure 6.9.

Table 6.10: Flutter speed analysis with $\left[45_{2 n} / 0_{n}\right]_{s}$ laminate

\begin{tabular}{cccccc}
\hline & \multicolumn{5}{c}{ Flutter Speeds (m/s) } \\
Material & $n$ & $c / t$ & HSDT & FSDT & \% Diff. \\
\hline 1 & 1 & 100 & 29.286 & 29.286 & $0.0000 \%$ \\
& 2 & 50 & 79.205 & 79.206 & $0.0004 \%$ \\
& 4 & 25 & 208.069 & 208.071 & $0.0013 \%$ \\
\hline 2 & 1 & 100 & 24.362 & 24.362 & $0.0000 \%$ \\
& 2 & 50 & 66.653 & 66.653 & $0.0006 \%$ \\
& 4 & 25 & 178.768 & 178.772 & $0.0022 \%$ \\
\hline 3 & 1 & 100 & 20.972 & 20.972 & $0.0001 \%$ \\
& 2 & 50 & 57.359 & 57.360 & $0.0005 \%$ \\
& 4 & 25 & 156.680 & 156.684 & $0.0027 \%$ \\
\hline
\end{tabular}

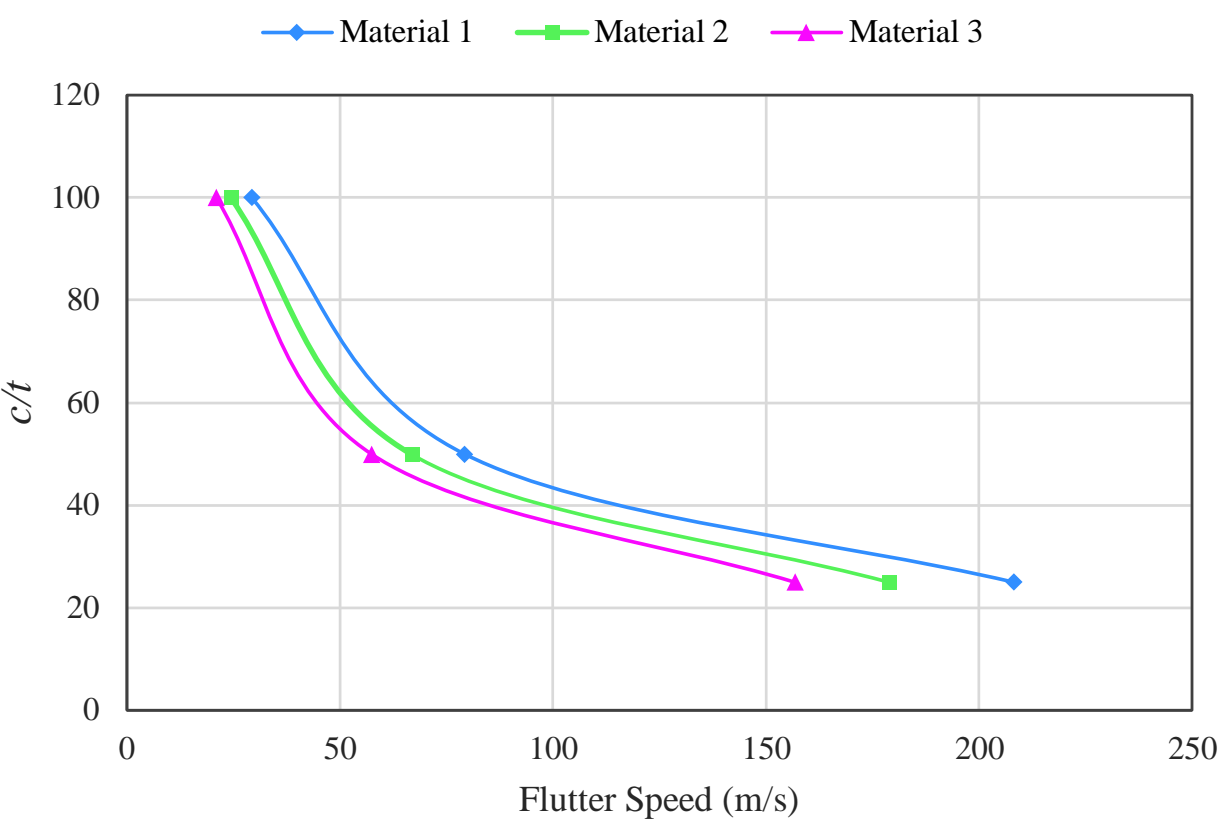

Figure 6.9: HSDT flutter speed analysis with $\left[45_{2 n} / 0_{n}\right]_{s}$ laminate 
The results of the $\left[45_{2 n} / 0_{n}\right]_{s}$ laminate analysis are similar to those of the $\left[0_{2 n} / 90_{n}\right]_{s}$ laminate. The difference between HSDT and FSDT is negligible and the flutter speeds are found to be highest in Material 3 and lowest in Material 1 for the same reasons as before. The introduction of the $45^{\circ}$ angle plies significantly increased flutter speeds compared to the cross-ply laminate. This phenomenon is explored in detail when the effect of ply orientation is analyzed in the next section.

Divergence and flutter speeds have been analyzed for three different materials with both HSDT and FSDT while varying plate chord-to-thickness ratio. The increase in laminate thickness expectedly increases the divergence and flutter speeds significantly, while the composite materials with relatively lower shear moduli experienced lower aeroelastic speeds. Although the application of HSDT increased divergence and flutter speeds, the percent difference in results between the two shear deformation theories is merely on the order of $1 \times 10^{-4}$ and $1 \times 10^{-3}$. These results overwhelmingly lead to the conclusion that the difference in flat plate divergence and flutter speeds between HSDT and FSDT are negligible. 


\subsection{Effect of Ply Orientation}

The primary benefit of composite materials within the field of aeroelasticity is the material coupling they exhibit, which is readily manipulated to achieve an optimized aeroelastic structure in the process of aerolelastic tailoring. This tailoring is performed based on a thorough understanding of how composite parameters such as layup, ply orientation, etc. influence aeroelastic performance. In support of this understanding, the present section studies the effect of ply orientation on the divergence and flutter speeds and frequencies of composite plates using the MONNA HSDT plate element.

The plate used for this analysis has the same geometry and orientation as that shown in Figure 6.5 of Section 6.2. Here, the composite material properties are those of Material 1 from Table 6.6 of the same section, which are representative of a common graphite-epoxy. In summary, the rectangular plate wing has properties listed in Table 6.11, where $s$ is the half-span.

Table 6.11: Composite plate properties for ply orientation analysis

\begin{tabular}{ll}
\hline Material Properties & \\
\hline$E_{1}(\mathrm{GPa})$ & 130 \\
$E_{2}(\mathrm{GPa})$ & 10.4 \\
$G_{12}(\mathrm{GPa})$ & 5.2 \\
$G_{13}(\mathrm{GPa})$ & 5.2 \\
$G_{23}(\mathrm{GPa})$ & 3.1 \\
$\nu_{12}$ & 0.28 \\
$\rho\left(\mathrm{kg} / \mathrm{m}^{3}\right)$ & 1600 \\
\hline $\mathrm{Geometric}$ Properties & \\
\hline$s(\mathrm{~m})$ & $0.24 \mathrm{~m}$ \\
$c(\mathrm{~m})$ & $0.06 \mathrm{~m}$ \\
$t_{p}(\mathrm{~m})$ & $0.1 \times 10^{-3}$ \\
$A R$ & 8 \\
\hline
\end{tabular}

The flutter and divergence speeds are analyzed for the $\left[\theta_{2}, 0\right]_{s}$ laminate where $\theta$ varies from $0^{\circ}-180^{\circ}$. The divergence and flutter speeds are nondimensionalized, as done by Ref. [8], with a flutter speed, $U_{R}$, at $\theta=0^{\circ}$. The flutter frequencies are similarly nondimensionalized by the flutter frequency, $\omega_{R}$, at $\theta=0^{\circ}$. The DPM aerodynamic model is employed for both flutter and divergence analyses, and there are $8 \times 12$ aerodynamic elements and $4 \times 6$ structural elements consistently applied throughout the analysis.

Figure 6.10 displays the nondimensional flutter and divergence speeds as a function of ply orientation. These results are similar to those produced by references in literature who have studied this behavior with CLPT and FSDT composite plate 


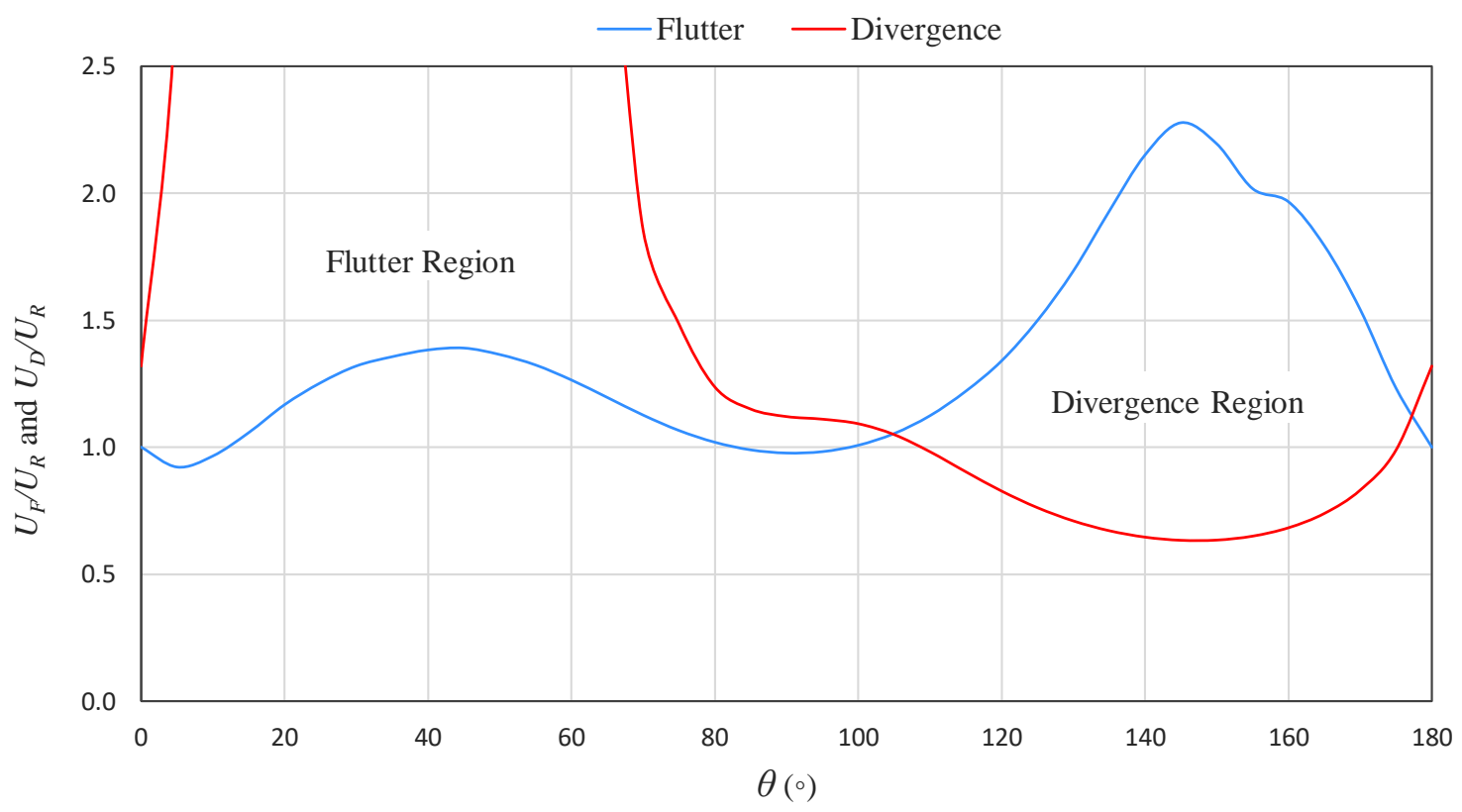

Figure 6.10: Nondimensional flutter and divergence speeds vs. ply orientation for $\left[\theta_{2} / 0\right]_{s}$ laminate

theories. Figure 6.10 reveals how the dominant aeroelastic instability phenomenon depends on ply orientation. The ply angle range, $\theta=0-105^{\circ}$, is considered the "flutter region" because the plate ultimately undergoes flutter behavior with increased freestream speed at these orientations. The flutter speed may be postponed at certain ply angles within this region relative to the $\theta=0^{\circ}$ laminate, where the maximum delay occurs at $\theta=45^{\theta}$. The ply angle range, $\theta=105-175^{\circ}$, is considered the "divergence region" because the plate ultimately diverges with increased freestream speed. Although the maximum flutter boundary occurs within this region, the plate diverges such that its not possible to take advantage of the highest flutter speeds. Similarly, the maximum divergence boundary occurs within the flutter region, but the plate flutters such that it's not possible to take advantage of the highest divergence speeds. This tradeoff exists because the composite directional stiffness either produces a wash-in structure that favors flutter delay or a wash-out structure that favors divergence delay [7].

Despite this inefficiency, there are nevertheless ply orientations within the flutter region where both flutter and divergence boundaries are higher than the $\theta=0^{\circ}$ laminate reference. It's important to note the behavior of these plots may vary significantly based on wing aspect ratio, sweep angle, representation etc. [7] [8]. Overall, the aeroelastic behavior of plate wings may be improved or worsened based on composite ply orientation, thus, meticulous analysis is required to capitalize on the benefits of aeroelastic tailoring.

Figure 6.11 displays nondimensional flutter and natural frequencies as a function of ply orientation. Again, these results are generally in agreement with those produced in literature. The flutter frequencies are in between the $1^{\text {st }}$ and $2^{\text {nd }}$ natural 


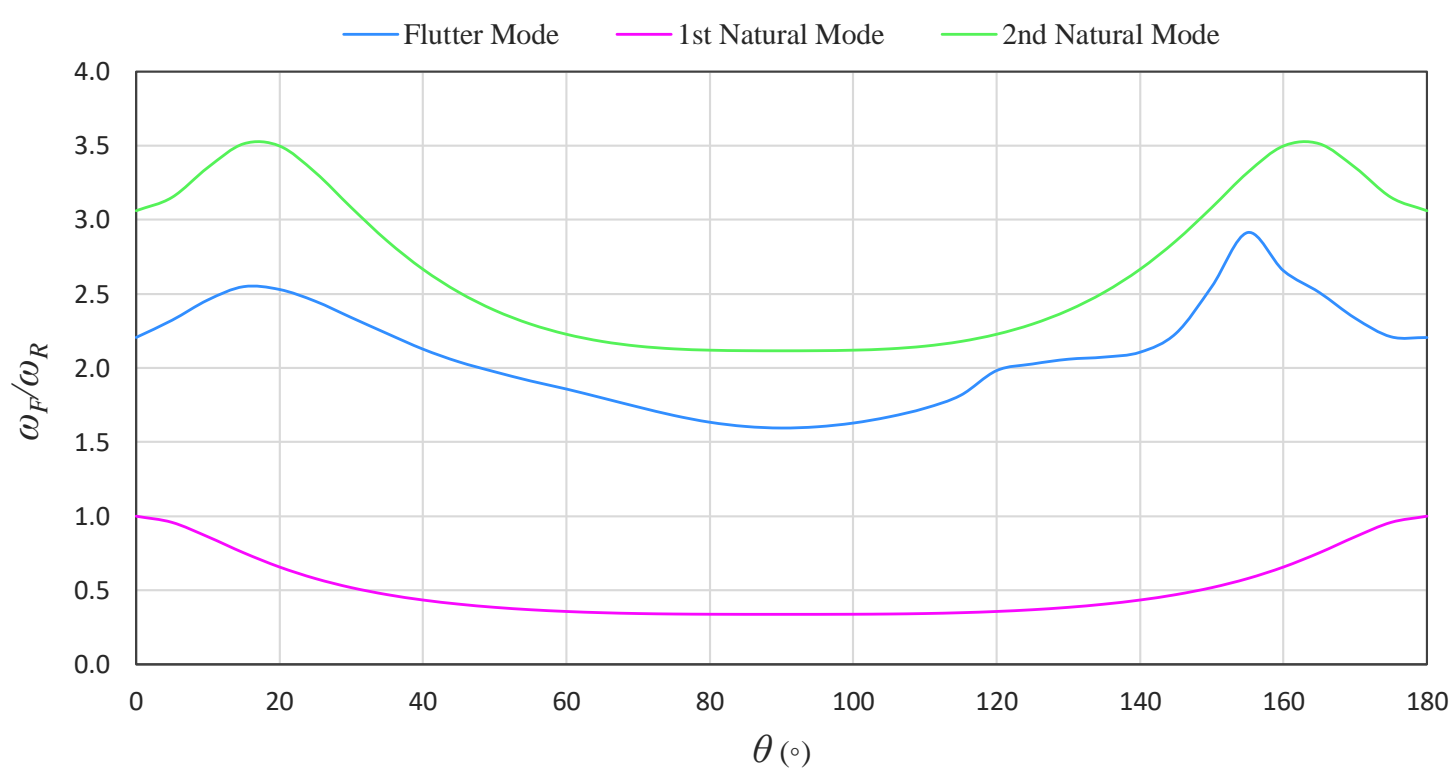

Figure 6.11: Nondimensional flutter and natural frequencies vs. ply orientation for $\left[\theta_{2} / 0\right]_{s}$ laminate

frequencies, which are the plate's first bending and torsional modes, respectively. Unlike the natural frequencies, the flutter frequencies are not symmetric with respect to ply orientation. The natural frequencies are symmetric because they are independent of the direction of a specific ply orientation. However, the flutter frequencies are asymmetric because of asymmetric aerodynamic loading. Reference [7] attributed this aerodynamic loading asymmetry to the Kutta condition at the trailing edge of the wing. 


\subsection{Effect of Aspect Radio}

The effect of aspect ratio on the flutter and divergence speeds of composite plates are analyzed in this section using the MONNA HSDT plate element and DPM aerodynamic model. This investigation is performed on a plate made with the graphite-epoxy material properties listed in Table 6.11 of Section 6.3, where the total wing area is consistently $S_{w}=0.0288 \mathrm{~m}^{2}$ and the ply thickness is $t_{p}=0.1 \times 10^{-3}$. The aspect ratio is defined traditionally, which is with respect to the entire wing span.

Nondimensional divergence velocities as a function of aspect ratio for symmetric $\left[-45_{2} / 0\right]_{s}$ and unsymmetric $[0 /-45]_{3}$ laminates are plotted in Figure 6.12. The divergence

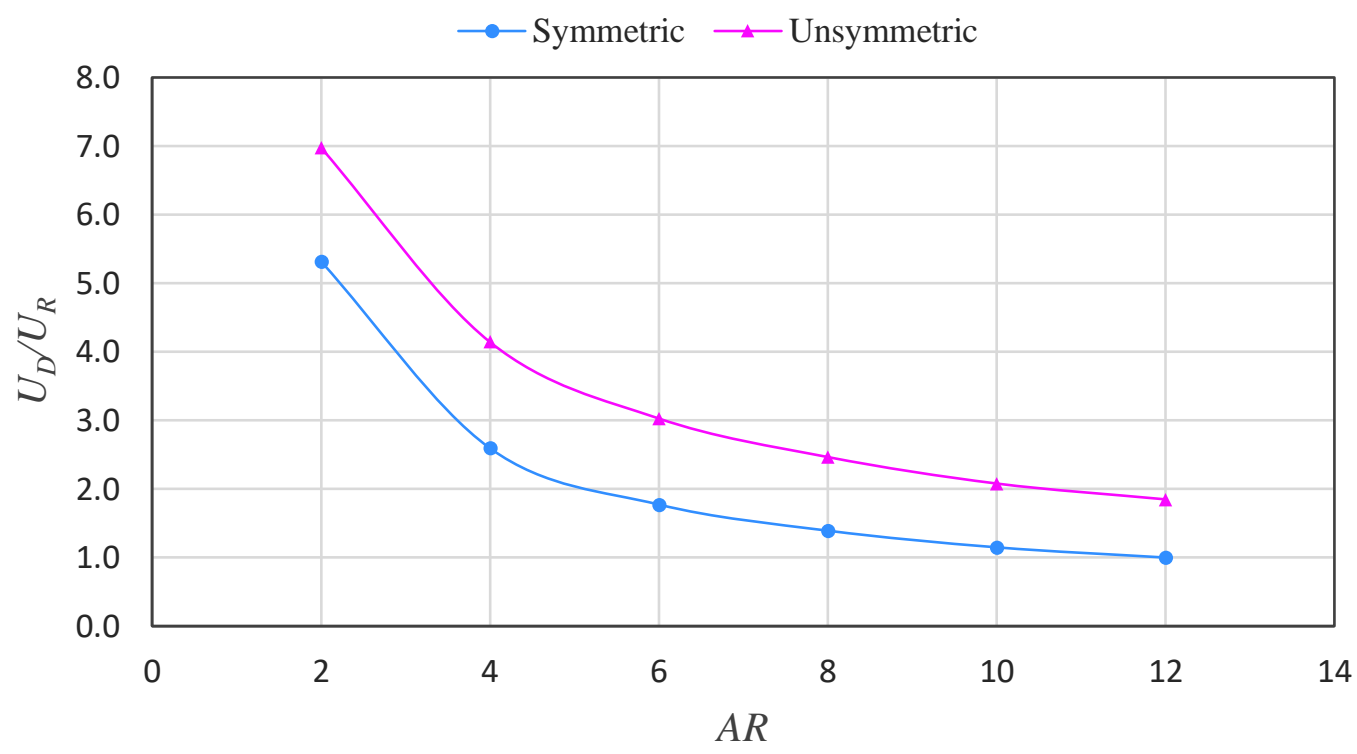

Figure 6.12: Divergence speeds vs. aspect ratio for $\left[-45_{2} / 0\right]_{s}$ and $[0 /-45]_{3}$ laminates

speeds are nondimensionalized with the reference divergence speed, $U_{R}$, analyzed with the symmetric laminate at $A R=12$. Nondimensional flutter velocities as a function of aspect ratio for symmetric $\left[45_{2} / 0\right]_{s}$ and unsymmetric $[0 / 45]_{3}$ laminates are plotted Figure 6.13. Flutter speeds are similarly nondimensionalized. For both analyses, the structural and aerodynamic elements are steadily decreased chordwise and increased spanwise as aspect ratio is increased.

The divergence speeds in Figure 6.12 are inversely related to wing aspect ratio. The symmetric laminate experienced lower speeds relative to the unsymmetric laminate, however, both responded in the same manner to increased aspect ratio. Similarly, the flutter speeds in Figure 6.13 are inversely related to wing aspect ratio. The symmetric laminate experienced lower speeds than the unsymmetric laminate at low aspect ratios, but both laminates experienced similar flutter speeds with increased aspect ratio.

The decrease in speed for both aeroelastic phenomena with increased aspect ratio is expected because both twisting and bending deflection are higher for increased cantilever plate length. Additionally, the torsional rigidity and bending stiffness are 


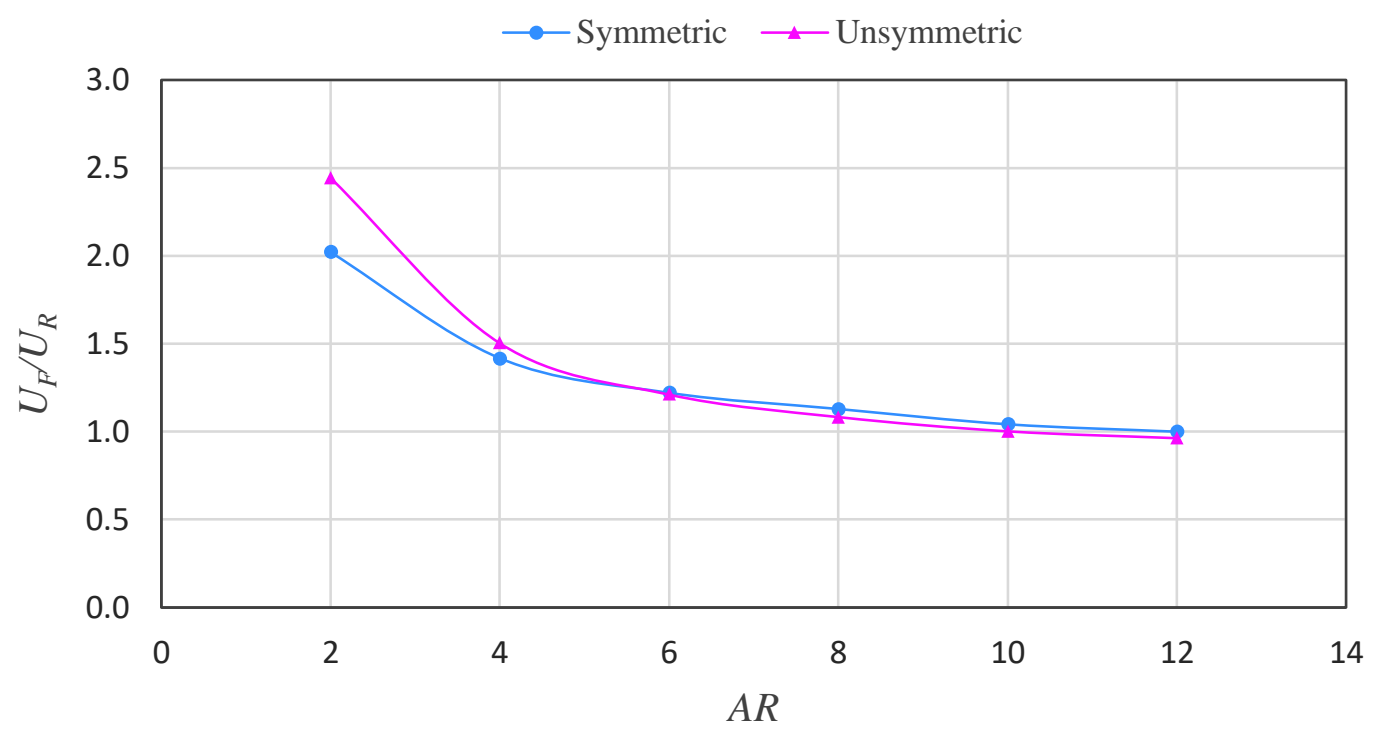

Figure 6.13: Flutter speeds vs. aspect ratio for $\left[45_{2} / 0\right]_{s}$ and $[0 / 45]_{3}$ laminates

influenced by the cross sectional chord length, which results in lower aeroelastic speeds for decreased chord length. 


\subsection{Effect of Composite Moduli Ratios}

The effect of composite moduli ratios on plate divergence and flutter speeds are analyzed in the present section with the MONNA HSDT element and DPM aerodynamic model. This investigation tests the longitudinal to transverse elastic modulus ratio, $E_{1} / E_{2}$, and the in-plane shear modulus to transverse elastic modulus ratio, $G_{12} / E_{2}$.

The composite plate in this analysis has the same material and geometric properties as those listed in Table 6.11 of Section 6.3 unless otherwise altered to vary moduli ratios. The $E_{1} / E_{2}$ ratio is varied by first letting $E_{1}=E_{2}$ and then steadily increasing $E_{1}$ to achieve the desired ratios. Similarly, the $G_{12} / E_{2}$ ratio is varied by first setting $G_{12}=0.4 E_{2}$ and then steadily increasing $G_{12}$. In each test, $4 \times 6$ structural elements and $8 \times 12$ aerodynamic elements are used. Divergence speeds are nondimensionalized by reference a divergence speed analyzed with a cross-ply laminate when the longitudinal and transverse elastic moduli are equal. Flutter speeds are nondimensionalized with a similarly analyzed reference flutter speed.

\section{Divergence Analysis}

The variation in plate divergence speeds with varying elastic moduli ratio is shown in Figure 6.14 for a variety of laminates. The divergence speed steadily increases with

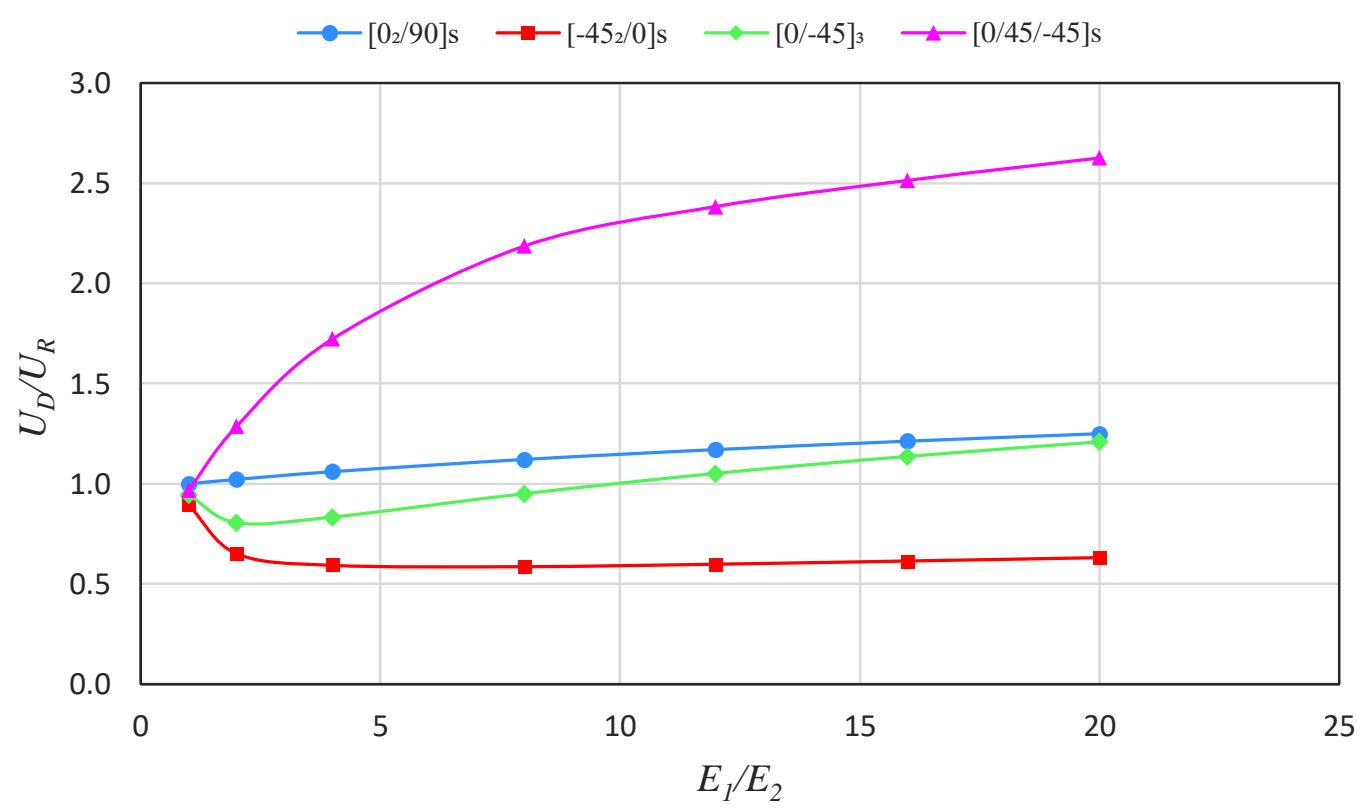

Figure 6.14: Divergence speeds vs. $E_{1} / E_{2}$ for various laminates

increased ratio in the $\left[0_{2} / 90\right]_{s}$ laminate, whereas the speeds initially decreased and then increased for the $\left[-45_{2} / 0\right]_{s}$ and $[0 /-45]_{3}$ laminates. The divergence boundary was most the improved in the $[0 / 45 /-45]_{s}$ laminate, which experienced the greatest change in 
speed among the tested laminates.

The variation in divergence speeds with varying in-plane shear to transverse elastic modulus ratio is shown in Figure 6.15. In this case, increasing $G_{12}$ caused the

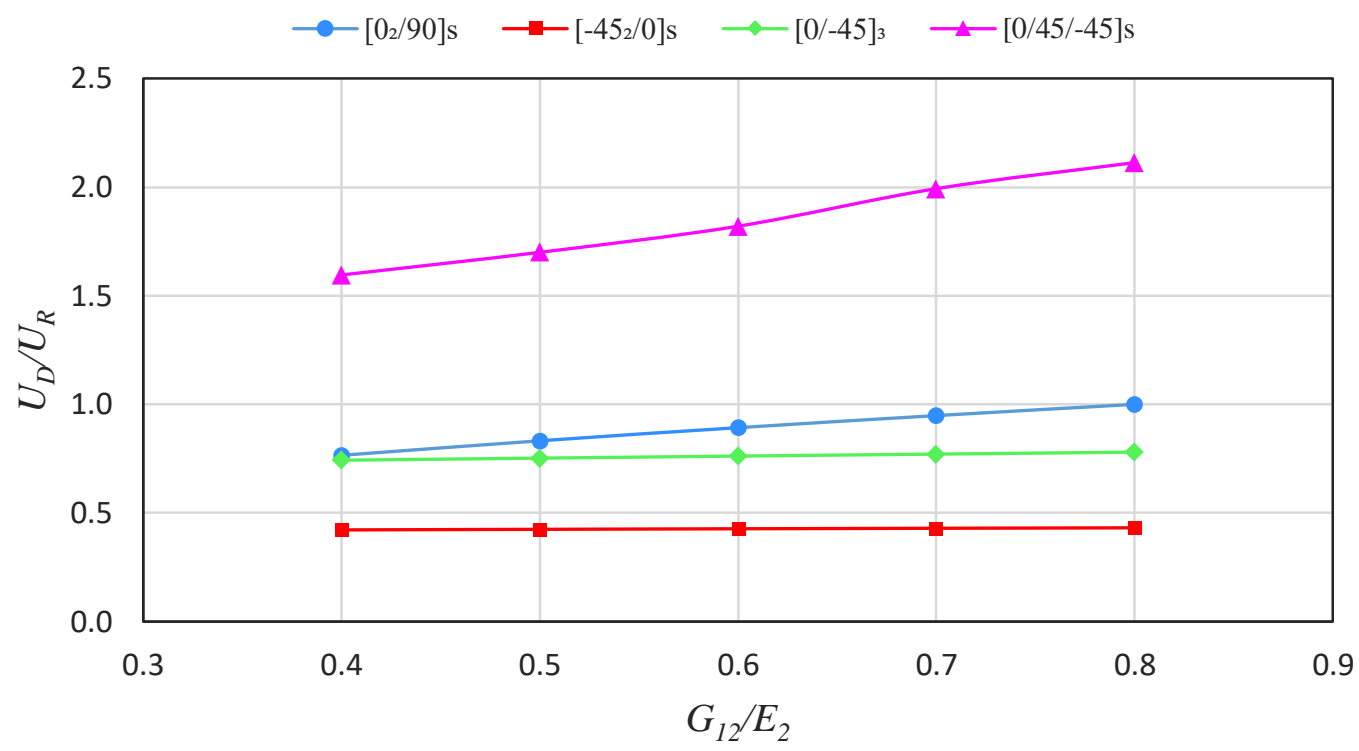

Figure 6.15: Divergence speeds vs. $G_{12} / E_{2}$ for various laminates

same effect in all laminates, however, the effect is more pronounced in some more than others. For example, the $\left[-45_{2} / 0\right]_{s}$ experienced a mere $2.3 \%$ increase in divergence speed, while the $[0 / 45 /-45]_{s}$ laminate experienced a $32.4 \%$ increase. Including positive angles in laminates has been shown to create wash-out structures that favor divergence delay in Section 6.3. Thus, the present results reveal an increased divergence sensitivity to in-plane shear moduli in a wash-out structure.

\section{Flutter Analysis}

The variation in plate flutter speeds with varying elastic moduli ratio is shown in Figure 6.16 for a variety of laminates. The influence of elastic moduli ratio is noticeably different in flutter. The cross-ply laminate, for example, experienced an initial decrease and then increase in flutter speed for increased moduli ratio, and all other laminates tested in the flutter analysis experienced a continuous increase in flutter speed. The speeds for the $\left[45_{2} / 0\right]_{s}$ and $[0 / 45]_{3}$ laminates vary parabolically, while the $[0 / 45 /-45]_{s}$ laminate demonstrates linear behavior.

The variation in flutter speeds with varying in-plane shear to transverse elastic modulus ratio is shown in Figure 6.17. All laminates experience increased flutter speeds for increased moduli ratio with the exception of the $\left[45_{2} / 0\right]_{s}$ laminate. When all laminates are prescribed the same in-plane shear moduli in Figure 6.16, the laminates with angle plies demonstrated high flutter sensitivity to increased longitudinal elastic modulus, whereas the cross-ply demonstrated low sensitivity. This is because laminates 


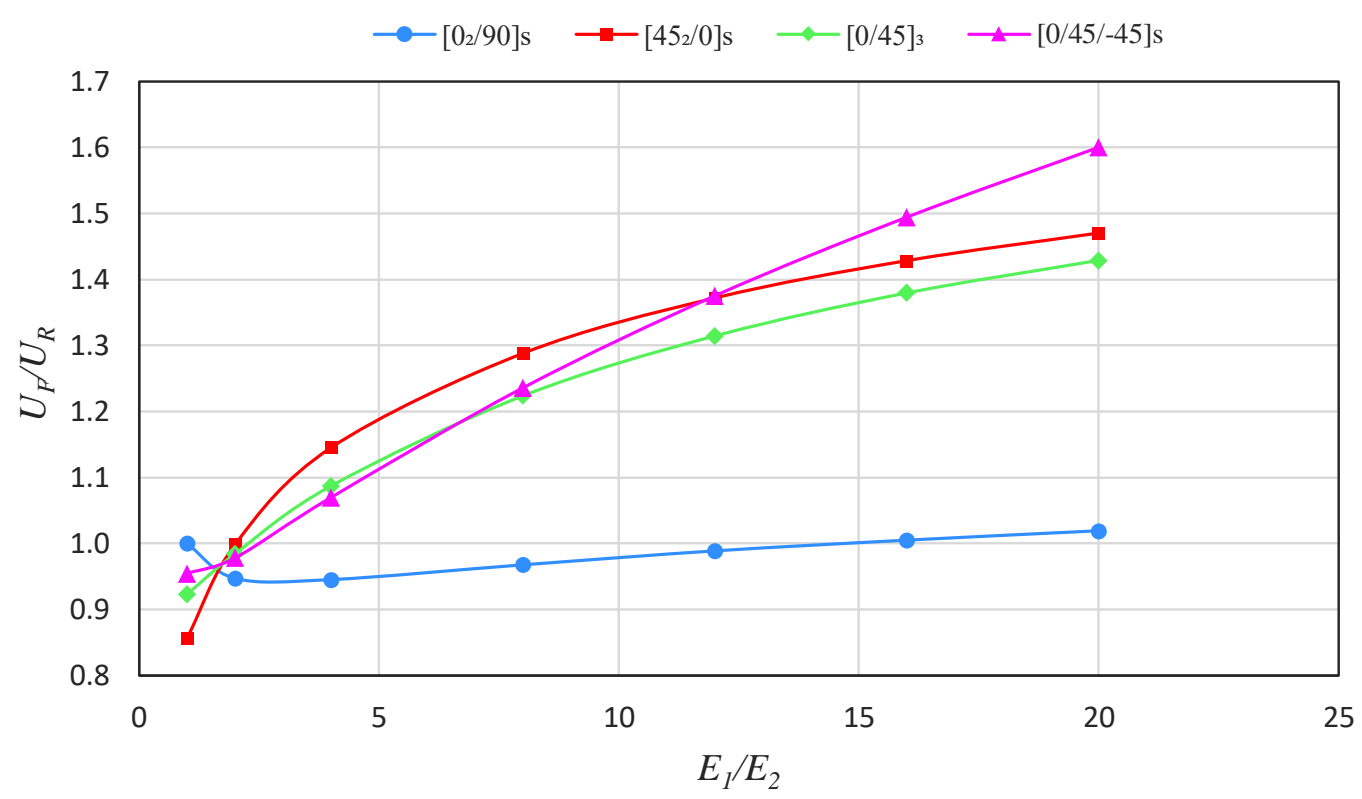

Figure 6.16: Flutter speeds vs. $E_{1} / E_{2}$ for various laminates

with angle plies have a need for longitudinal stiffness to resist bending and delay flutter, while the cross-ply laminates already have high stiffness in the longitudinal direction. When all laminates are prescribed the same elastic moduli in Figure 6.17, the laminates

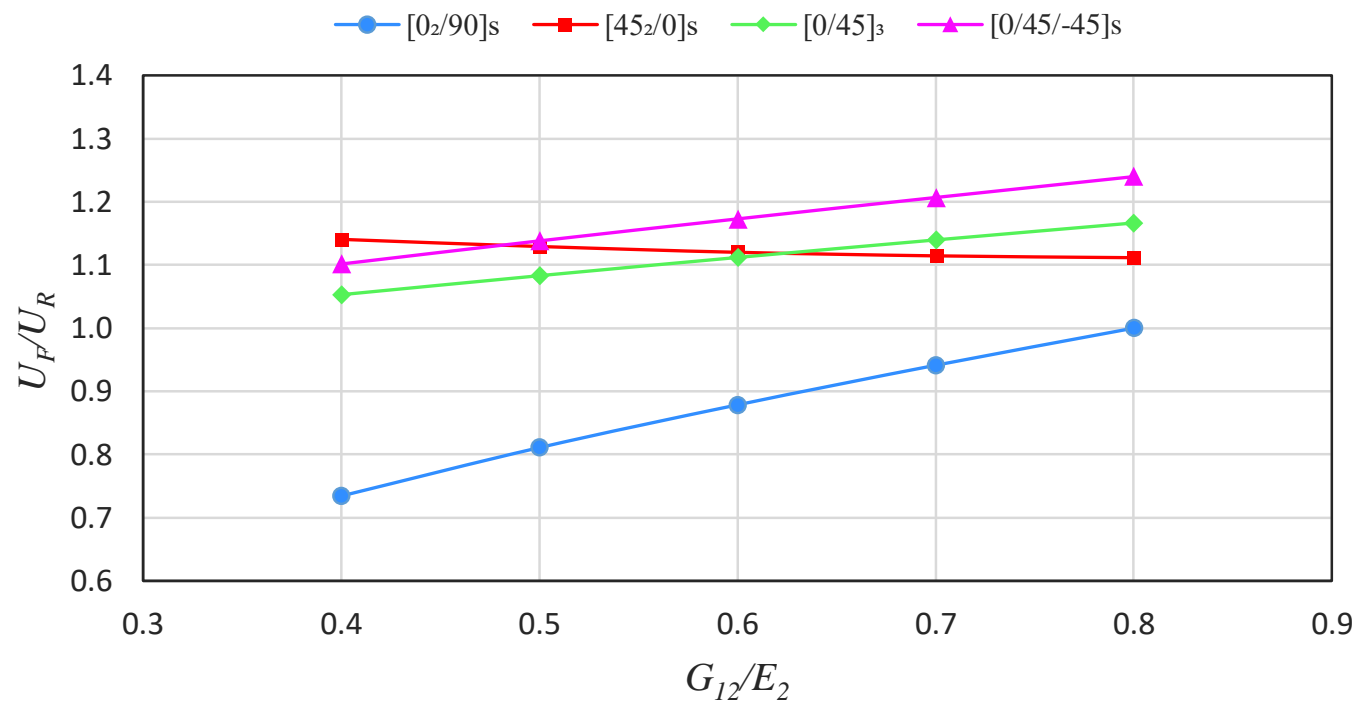

Figure 6.17: Flutter speeds vs. $G_{12} / E_{2}$ for various laminates

with angle plies demonstrate low flutter sensitivity to increased in-plane shear moduli, however, the cross-ply laminate demonstrates high sensitivity. This is because angle plies provide torsional rigidity, thus, the cross-ply laminate has a need for increased torsional rigidity to resist twist and delay flutter, whereas the angled laminates already have high torsional rigidity. Overall, the effect of moduli ratios on the divergence and flutter characteristics of composite plates is significant and depends greatly on laminate layup and ply orientation. 


\section{Chapter 7}

\section{Conclusions and Recommendations}

\section{Conclusions}

Based on the research conducted in this thesis, the following summary of conclusions are made:

- A new conforming higher-order $h$ - $p$-version plate finite element, called MONNA, has been successfully developed and implemented in MATLAB to investigate the divergence and flutter characteristics of composite plate wings.

- The MONNA plate element is conforming; however, it does not directly have the capacity for quadrilateral transformation similar to other $C^{1}$-continuous rectangular plate elements.

- When comparing with experimental results from other authors, the Doublet Lattice Method provided the most accurate composite plate divergence speeds, while the Doublet Point Method provided the most accurate flutter speeds.

- Some composite aeroelasticity researchers have applied the aerodynamic loading in a manner that is not consistent with the Doublet Lattice Method. The aerodynamic loads should be applied as uniform line loads across the panel quarter chords.

- The conforming $h-p$ version MONNA element provided better aeroelastic convergence compared with traditional rectangular $C^{1}$-continuous plate elements. 
- The differences between FSDT and HSDT in aeroelastic analysis are negligible even for thick composite plate wings where $c / t=25$. This leads to the conclusion that HSDT is superfluous in aeroelastic plate analysis, which previous aeroelasticity researchers who have employed HSDT have neglected to establish.

- A parametric study has been successfully performed on the divergence and flutter of composite plates using the MONNA plate element. This has verified the divergence-flutter trade-off associated with change in ply orientation as well as other insights into aeroelastic tailoring. Aeroelastic speeds can vary significantly with composite laminate layup and ply orientation.

- Because HSDT usually requires $C^{1}$ continuity and rectangular $C^{1}$ plate elements have difficulty with quadrilateral transformation, rectangular HSDT plate elements are difficult to employ in the field of aeroelasticity.

- Although the practicality of the developed higher-order plate element is limited within the field of aeroelasticity, it may be useful in other engineering applications.

\section{Recommendations for Future Research}

1. The aeroelasticity of composite box beams could be analyzed to check the need for HSDT in box-beam wings.

2. A more rigorous comparison could be made between the MONNA plate element and other elements with respect to approximating structural deflections, stresses, natural frequencies, and buckling loads.

3. The MONNA plate element could be tested in other engineering applications where elements that can produce highly accurate plate deflections or stresses are needed. 


\section{References}

[1] Smith, F. "The Use of Composites in Aerospace: Past, Present, and Future Challenges". PowerPoint file. 2013.

[2] Wright, J. R. and Cooper, J. E. Introduction to Aircraft Aeroelasticity and Loads. John Wiley \& Sons Ltd., 2007.

[3] Megson, T. H. G. Aircraft Structures for Engineering Students. 4th. Elsevier Ltd., 2007.

[4] Banerjee, J. R., Su, H., and Jayatunga, C. "A dynamic stiffness element for free vibration analysis of composite beams and its application to aircraft wings". In: Computers $\&$ Structures 86.6 (2008). Civil-Comp Special Issue, pp. 573-579.

[5] Babuška, I. and Guo, B.Q. "The $h, p$ and $h-p$ version of the finite element method; basis theory and applications". In: Advances in Engineering Software 15.3 (1992), pp. 159-174.

[6] Hollowell, S. J. and Dugundji, J. "Aeroelastic flutter and divergence of stiffness coupled, graphite/epoxy cantilevered plates". In: Journal of Aircraft 21.1 (1984), pp. 69-76.

[7] Lin, K., Lu, P., and Tarn, J. "Flutter analysis of cantilever composite plates in subsonic flow". In: AIAA Journal 27.8 (1989), pp. 1102-1109.

[8] Koo, K. N. and Lee, I. "Aeroelastic behavior of a composite plate wing with structural damping". In: Computers and Structures 50.2 (1994), pp. 167-176.

[9] Chattopadhyay, A., Zhang, S., and Iha, R. "Structural and aeroelastic analysis of composite wing box sections using higher-order laminate theory". In: 37th Structure, Structural Dynamics and Materials Conference. 1996.

[10] Koo, K. N. "Aeroelastic Characteristics of Double-Swept Isotropic and Composite Wings". In: Journal of Aircraft 38.2 (2001), pp. 343-348.

[11] Kameyama, M. and Fukunaga, H. "Optimum design of composite plate wings for aeroelastic characteristics using lamination parameters". In: Computers and Structures 85.3 (2007), pp. 213-224. 
[12] Abbas, M., Negm, H., and Elshafei, M. "Flutter and Divergence Characteristics of Composite Plate Wing". In: International Conference on Aerospace Sciences and Aviation Technology 15 (2013), pp. 1-21.

[13] Mahran, M., Negm, H., and Elsabbagh, A. Aeroelastic analysis of plate wings using the finite element method. Lambert Academic Publishing, Feb. 2015.

[14] Mahran, M., Elsabbagh, A., and Negm, H. "A comparison between different finite elements for elastic and aero-elastic analyses". In: Journal of Advanced Research 8.6 (2017), pp. 635-648.

[15] Phan, N. D. and Reddy, J. N. "Analysis of laminated composite plates using a higher-order shear deformation theory". In: International Journal for Numerical Methods in Engineering 21.12 (1985), pp. 2201-2219.

[16] Thai, C. H. et al. "Analysis of laminated composite plates using higher-order shear deformation plate theory and node-based smoothed discrete shear gap method". In: Applied Mathematical Modelling 36.11 (2012), pp. 5657-5677.

[17] Reddy, J. N. An Introduction to the Finite Element Method. 3rd. McGraw Hill, 2006.

[18] Reddy, J. N. Mechanics of Laminated Composite Plates and Shells. 2nd. CRC Press, 2004.

[19] Sivaneri, N. Flutter Analysis of Composite Wings using HSDT and Higher-Order FEM. Tech. rep. AFRL SFFP, Aug. 2019.

[20] Sheikh, A. H. and Chakrabarti, A. "A new plate bending element based on higher-order shear deformation theory for the analysis of composite plates". In: Finite Elements in Analysis and Design 39.9 (2003), pp. 883-903.

[21] Melosh, R. J. "Basis of Derivation of Matrices for the Direct Stiffness Method". In: AIAA Journal 1.7 (1963), pp. 1631-1637.

[22] Bogner, F. K., Fox, R. L., and Schmidt, L. A. The Generation of Inter-ElementCompatible Stiffness and Mass Matrices by the Use of Interpolation Formulas. Tech. rep. AFFDL-TR-66-80, Air Force Institute of Technology, WPAFB, 1965.

[23] Bertin, J. and Cummings, R. Aerodynamics for Engineers. 6th. Pearson, 2014.

[24] Hodges, D. H. and Pierce, G. A. Introduction to Structural Dynamics and Aeroelasticity. 2nd. Cambridge University Press, 2002.

[25] Albano, E. and Rodden, W. P. "A doublet-lattice method for calculating lift distributions on oscillating surfaces in subsonic flows." In: AIAA Journal 7.2 (1969), pp. 279-285. 
[26] Laschka, B. "Das Potential und das Geschwindigkeitsfeld der harmonisch schwingenden tragenden Fläche bei Unterschallströmung". In: ZAMM Journal of Applied Mathematics and Mechanics 43.7-8 (1963), pp. 325-333.

[27] Blair, M. A Compilation of Mathematics Leading to the Doublet Lattice Method. Tech. rep. WL-TR-92-3028, Air Force Wright Laboratory, WPAFB, 1992.

[28] Ueda, T. and Dowell, E. H. "A new solution method for lifting surfaces in subsonic flow". In: AIAA Journal 20.3 (1982), pp. 348-355.

[29] Eversman, W. and Pitt, D. M. "Hybrid doublet lattice/doublet point method for lifting surfaces in subsonic flow". In: Journal of Aircraft 28.9 (1991), pp. 572-578.

[30] Harder, R. L. and Desmarais, R. N. "Interpolation using surface splines." In: Journal of Aircraft 9.2 (1972), pp. 189-191.

[31] Aeroelastic Analysis User's Guide. Siemens. 2014.

[32] Brendel, M. and Sulaeman, E. "Improved doublet lattice formulation for calculating aerodynamic loads on lifting surfaces in unsteady subsonic flow". In: 12th Applied Aerodynamics Conference. 1994.

[33] Haught, J. Flutter Analysis of Composite Wings using HSDT and Higher-Order FEM. Tech. rep. AFRL SFFP, Aug. 2019.

[34] Barbero, E. J. Introduction to Composite Materials Design. 2nd. CRC Press, 2011. 


\section{Appendix A}

\section{Gauss Quadrature Integration}

The Gauss quadrature numerical integration technique is used in the present research to integrate the finite element stiffness and inertia matrices as well as the aerodynamic line loads in the DLM load transformation. The Gauss quadrature integration is performed by a weighted sum through a number of function values and corresponding weights within the domain. Numerical integration using $n$ sampling points is exact for polynomials of order $(2 n-1)$. The integration scheme in two dimensions may be represented as

$$
\int_{-1}^{1} \int_{-1}^{1} f(\xi, \eta) d \xi d \eta=\sum_{i=1}^{n} \sum_{j=1}^{m} w_{i} w_{j} f\left(a_{i}, b_{j}\right)
$$

where $a_{j}$ and $b_{j}$ are the $\xi$ and $\eta$ coordinates at the sampling points, respectively, and $w_{j}$ are the corresponding weights. In this study, a seven point Gauss quadrature scheme is used, where the sampling points and weights are presented in Table A.1 The finite

Table A.1: Gauss quadrature points and weights

\begin{tabular}{l|l} 
Sampling Points & Weights \\
\hline 0.0000000000000000 & 0.4179591836734694 \\
\pm 0.4058451513773972 & 0.3818300505051189 \\
\pm 0.7415311855993945 & 0.2797053914892766 \\
\pm 0.9491079123427585 & 0.1294849661688697
\end{tabular}

element stiffness and inertia matrices are simultaneously transformed into the physical domain with the Jacobian, $J$, while performing this numerical integration. Note that the DLM line loads are integrated only in the $\xi$ direction for the load transformation. 